\title{
Exploring the Scientific Philosophy and Application of the Langlands Program
}

\section{Circular Logarithm Algorithm: Unrelated Mathematical Models are Solved in the 0 to 1 Interval}

\author{
Yiping Wang \\ Association of Senior Citizens and Technicians, Quzhou, Zhejiang Province, China 324000 \\ wyp3025419@163.com
}

\begin{abstract}
I explore the scientific philosophy of the Langlands program."Mathematical relativity" I define arbitrary functions (groups, clusters, algebraic integer equations and circular logarithmic equations) as a finite number of non-repetitive combination-exchange-aggregation of infinite uncertain "element-factor". I prove symmetry and asymmetry, unity, isomorphism, relative symmetry, zero point, parallel/serial, equivalent permutation, normalization etc. of the Reciprocity Theorem. I establish circular Logarithmic equation with the eccentric elliptic function as base and perform arithmetic solution of the unrelated mathematical model within [0 to 1] interval. The Circular Logarithm Algorithm has extensive and close links with many fields, ranging from mathematical modeling of cosmic evolution, quantum computing, neuromorphic calculation and blockchain to the scientific field of architecture chip simplification.
\end{abstract}

Key words: Langlands Program; uncertainty; reciprocal theorem; "1" norm invariance; circular logarithmic equation;

\section{Foreword}

In 1667, Napier began the logarithm and established various disciplines and branches to promote the development of human science. Hilbert tried to unify the mathematics with Geometry. In 1931, Gödel's incompleteness theorem swayed its entire foundation. Klein said in Mathematical Thought: from Ancient to Modern Times ${ }^{[1]}$ : Mathematics has not made substantial progress since then.

In 1967 Langlands Program ${ }^{[2]}$ was proposed in the form of conjectures, which is a unified and universal point of view on many fields of modern mathematics and of great philosophical importance. In this paper $I$ intend to explore $\{X\}$ of group theory, algebra, geometry, number theory, as well as the uncertain topological, probabilistic, prime distribution, chaos as well as fractal automorphic functions, and figure out in what way a deep connection is built up through a special function.

For centuries, mathematicians have created many algorithms and mathematical ideas. The latest achievement was the cooperation between Hun Zhiwei from China and Wu Baozhu from Vietnam in 2015, which proved the Reciprocity Theorem of symmetry in the Langlands Program, or the discrete algorithm 
based on $\mathrm{W}(\bullet)=\mathrm{G}(\bullet) \mathrm{F}(\bullet)=1$, which is called Central Symmetric Elliptic Function. At present, discrete algorithm has been successfully solved, and the development of Von Neumann's Structural Digital Calculation has reached saturation. Yet so far no one has proved another Reciprocity Theorem of asymmetry in the Langlands Program: the entanglement algorithm based on $\mathrm{W}(\bullet)=\mathrm{G}(\bullet) \mathrm{F}(\bullet) \neq 1$ which is called Eccentric Asymmetric Elliptic Function and is more basic. Many scholars at home and abroad are actively exploring the entanglement calculation of asymmetry.

In this paper I propose a Circular Logarithm Algorithm, a special function that attempts to meet the requirements of the Langlands Program ${ }^{[3]}$.Defining functions (groups, algebraic geometric numerical equations, circular logarithmic equations) are infinite "element-factors" of uncertainty that are not repeated combinations-switch-topology-sets.Proof of symmetry, asymmetry, and relative symmetry of the reciprocity theorem as well as unity, isomorphism, symmetry, zero point, parallel/serial, equivalent permutation, normalization etc constitute the basic theorems of Circular Logarithm Algorithm. Integrate discrete and entangled algorithms into a self-consistent whole, and establish an abstract logarithmic equation irrelevant to mathematical models with quadratic circle function as its base, which is named as the circular logarithmic equation and the eigenmode function(ie: mean value of forward, neutral, and inverse functions) are arithmetically solved in the periodic closed interval [0 to 1]. It is called relativistic structure and supersymmetric unit matrix. This effort helps to revolve the traditional mathematical foundation and develop the unifying potential of mathematics.

Practical application examples: function unification, physical formula unification, three-body problem, natural force unification, quantum computing principle, digital simulation cosmological equation, parity non-conservation, etc. It reflects the extensive and self-consistent connection between circle logarithm and blockchain, artificial intelligence, neuromorphology, deformation and degeneration, vortex and radiation, structural simplification, and mathematical modeling.

\section{The Basic Definition}

Definition 1: Element $(\cdot)$ : Element has uncertainty, infinite single element - factor, which is a multiplication or addition of random and regular non-repetitive combinations. Element-factor characteristics can be "continuous and discontinuous, symmetrical and asymmetrical, uniform and nonuniform, sparse and non-sparse, random and regular, discrete and entangled, wave and grain, vortex and radiation". The combination of these is a combination of infinite combinations of $P= \pm 0, \pm 1, \pm 2, \ldots, \pm p$, ..., without repetition and any finite topology combination.

$$
\begin{aligned}
& \sum_{\mathrm{i}=\mathrm{p}}^{(S \pm N)}\left[x_{a}{ }^{K}, x_{b}{ }^{K}, \ldots, x_{p}{ }^{K}, \ldots\right\} \in\{X\}^{K(Z \pm S \pm N)} ; \\
& \left\{x_{p}\right\}^{K(S \pm N \pm p)}=\left\{\sum_{\mathrm{i}=p}^{(S \pm N)} \prod_{\mathrm{i}=\mathrm{p}}^{(S \pm N)}\left[x_{a}^{K(S \pm N \pm p \pm 0)}, x_{b}{ }^{K(S \pm N \pm p \pm 1)}, \ldots, x_{p}{ }^{K(S \pm N \pm p \pm p)}, \ldots\right\}^{K(S \pm N \pm p)} ;\right.
\end{aligned}
$$

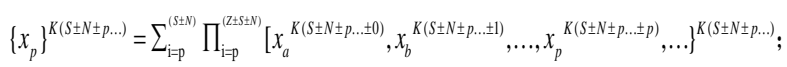

Where: variable element set $\left\{X_{p}\right\}^{K(Z \pm S \pm N \pm p \cdots)}$; infinite element power function: $(Z / t)=K(Z \pm S \pm N \pm P) / t$ any finite power dimension : $(S / t)=K(S \pm N \pm P) / t$; calculus order combination: $( \pm N)$; sub-item of the item sequence combination $( \pm \mathrm{P})$; function property $K=(+1,0,-1)$; kinetic time $(/ \mathrm{t}),\{\ldots\}$ any finite set; $\{\ldots, \ldots\}$ infinite set. (The same below). 
Definition 2: Function $\mathrm{W}(\bullet)$ : The infinite element is multiplied and joined to form a set-like function of any finite sub-item in an infinite program. There are high-order partial calculus equations or high-power polynomials.

$$
\begin{aligned}
& W(\cdot)=\sum_{(\mathrm{l}=S)}^{(Z \pm \pm N)}\left\{\prod_{(\mathrm{i}-\mathrm{p})}^{K(Z \pm \pm N)}\left[x_{a}^{K(S \pm N) / t}, x_{b}{ }^{K(S \pm N) / t}, \ldots, x_{p}{ }^{K(S \pm N) / t}, \ldots\right]\right\}^{K(Z \pm S \pm N) / t} \\
& =A x^{K(Z \pm S \pm N \pm 0) / t}+B x^{K(Z \pm S \pm N \pm 1) / t}+\ldots+P x^{K(Z \pm S \pm N \pm p) / t}+\ldots ;
\end{aligned}
$$

Where: $A, B, \ldots P, \ldots$ calculus equation coefficients, implicit element combination coefficient $\mathrm{C}_{(\mathrm{S} \pm \mathrm{N} \pm \mathrm{p})}$. Unknown independent variable $\{X\}^{K(Z \pm S-N-p) / t}$, known (target, prior) variable $\left\{X_{0}\right\}^{K(Z \pm S+N+p) / t}$.

Definition 3: eigenmode function $\mathrm{WO}(\bullet$ ) (ie: average of positive, medium, and inverse functions) infinite program combination form divided by combination coefficient, $A$ set of average values $\left\{X_{0}\right\}^{K(Z \pm S \pm N \pm p)}$ of each combined sub-item is obtained, which may also be represented by a matrix.

$$
\begin{aligned}
& W_{0}(\cdot)=\left\{X_{0}\right\}^{Z / t} \\
& =\left\{\sum_{(i=p)}^{(Z \pm S \pm N)}\left(1 / C_{(S \pm N \pm p)}\right)^{K} \prod_{(i=p)}^{K(S \pm N)}\left(X_{p}\right)^{K}+\ldots\right\}^{K(Z \pm S \pm N \pm p) / t} \\
& =\sum_{(i=p)}^{(Z \pm S \pm N)}\left\{X_{0}\right\}^{K(Z \pm S \pm N \pm p) / t}+\ldots ;
\end{aligned}
$$

Equation (1.3) reflects the invariant characteristics of its function (including mechanical parameters). Property power function: $\mathrm{K}=(+1,0,-1)$.

When: $\mathrm{K}=-1$, that is, the Riemann $\zeta$ function "the sum of the reciprocals and then the reciprocal", called the inverse eigenmode function.

$\mathrm{K}=+1$, that is, the Riemannian function "the sum of positive numbers and positive numbers" is called the forward eigenmode function.

$\mathrm{K}= \pm 1$, that is, the Riemann $\zeta$ function "sum of zero power and then zero power", called the zero point eigenmode function.

Definition 4: Relationship between the combination coefficient and the polynomial coefficient $(A, B, \ldots, P)$ :

Any finite element $\mathrm{K}(\mathrm{S} \pm \mathrm{N}) / \mathrm{t}$ regularization combination:

$\left\{X_{0}\right\}^{K(S \pm N \pm 0)}=\left(C_{(S \pm N \pm 0)}\right)=1, \quad(A=1)$; infinite elements have no regularity Coefficient, the combination coefficient of the first term $C_{(Z \pm S \pm N \pm 0)}=C_{(Z \pm S \pm N \pm p)} \neq 1$;

$$
\begin{array}{r}
C_{(S \pm N \pm p)}=(S \pm N \pm 0) \ldots(S \pm N \pm p) ! /(p \pm 1)(p \pm 0) \ldots 1 ! \\
\left\{X_{\mathbf{o}}\right\}^{K(S \pm N \pm 0)}=A / C_{(S \pm N \pm 0)} ; \\
\left\{X_{\mathbf{o}}\right\}^{K(S \pm N \pm 1)}=\boldsymbol{B} / C_{(S \pm N \pm 1)} ; \ldots ; \\
\left\{X_{\mathbf{o}}\right\}^{K(S \pm N \pm p)}=\boldsymbol{P} / C_{(S \pm N \pm p)} ; \ldots ; \\
\sum_{(i=p)}^{(Z \pm S \pm N)} C_{(S \pm N \pm p)}=\{2\}^{(Z \pm S \pm N \pm p)} ;
\end{array}
$$

In the formula: (! factorial). The sum of the combination coefficients, physics is called qubit.

Definition 5: Circular logarithmic equation and expansion: After extracting the eigenmode function, the element-factor has a common quadratic eccentric ellipse infinite set and arbitrary finite topological variation rules, and an elliptic function with no specific element and irrelevant mathematical model is 
established. The logarithm of the base $\left(1-\eta^{2}\right)^{K(Z / t)}$ is expanded to obtain a complete set of isomorphic topologies, probabilities, chaos, fractals, exchanges, zeros, and so on.

$$
\left(1-\eta^{2}\right)^{K(Z) / t}=\sum_{(i=p)}^{(Z \pm S \pm N)}\left(1-\eta^{2}\right)^{K(Z \pm S \pm N \pm p) / t}
$$

Definition 6: The circular logarithmic equation defines the domain [0 to 1] and [0 or 1]: the function of each sub-item introduces the principle of group relativity, and the analytical mapping is symmetric and asymmetrical elliptic functions in the periodic closed interval [0 to 1 ] The superposition inside.

$$
\{0\} \leq \sum_{(i=p)}^{(Z \pm S \pm p) / t}\left(1-\eta^{2}\right)^{K(Z \pm S \pm N \pm p) / t} \leq\{1\}
$$

(a) A eigenmode function corresponding to a set of infinite rational points (ie, mean values of positive, middle, and inverse functions)

$$
\sum_{\mathrm{i}=\mathrm{p}}^{(Z \pm S \pm N)}\left(1-\eta^{2}\right)^{K(Z \pm S \pm N) / t}=[\operatorname{Oor} 1]
$$

(b) A regularization combination of topologies and probabilities corresponding to any finite non-repetitive positive, medium, and inverse functions.

$$
\sum_{(i= \pm p)}^{(S \pm N)}\left(1-\eta^{2}\right)^{K(S \pm N \pm p) / t}=[0 t o 1]
$$

Definition 7: Define the principle of equivalent substitution (covariation principle): the entangled elements (groups, clusters, quantitative particles) whose combined energy, orbit, frequency, wave, .... In the invariant eigenmode form, the function changes have the same reciprocal change and construction for each element-factor. Any one or more of the element states and rules may be equivalently replaced with each other. The principle of equivalent replacement (covariation principle). However, elements of discrete states (groups, clusters) cannot be applied because they have no interaction.

$$
\left\{X_{p}\right\}_{(i=p)}^{K(Z \pm S \pm N+p) / t}=\left(1-\eta^{2}\right)^{K(Z \pm S \pm N \pm p) / t}\left\{X_{0 p}\right\}^{K(Z \pm S \pm N+p) / t} ;
$$

Definition 8: The calculus symbol (including the logical algebraic sign) is changed to the polynomial power function $\mathrm{K}( \pm \mathrm{S} \pm \mathrm{N} \pm \mathrm{P}) / \mathrm{t}$.

Differential: $(\mathrm{d}, \partial)(\mathrm{S}$ power original function) reduced order $(\mathrm{S}-\mathrm{N})$ and descended $(-\mathrm{P})$ :

$$
W(\cdot)^{(S-N)}=\partial^{n}\left\{X^{n}\right) / \partial t^{n}=d^{n}\left\{X^{n}\right) / d t^{n}=\{X)^{K(Z \pm S-N-p) / t} ;
$$

Integral: $\left(\mathrm{J}^{\mathrm{n}}\right)$ (S power original function) ascending order $(\mathrm{S}+\mathrm{N})$ and increasing order $(+\mathrm{P})$ :

$$
W(\cdot)^{(S+N)}=\int^{n}\left\{X^{n}\right) \partial^{n} t=\int^{n}\left\{X^{n}\right) d^{n} t=\{X)^{K(Z \pm S+N+p) / t} ;
$$

Where: combination coefficient: integral $\left(1 / C_{(S+N+p)}\right)^{+1}$; differential $\left(1 / C_{(S+N+p)}\right)^{-1}$; original function $\left(1 / C_{(S \pm N \pm p)}\right)^{ \pm 0}$.

Definition 9: Define circular log normalization: a nonlinear combination of arbitrary dimensions, eliminating the repeated combination repetition rate $f_{p}$ into a linear equation.

$$
\begin{aligned}
& F(\cdot) G(\cdot)=\left\{\left(X_{p}\right)^{K}+\ldots\right\}^{K(Z \pm S \pm N \pm p) / t}=\rightarrow \sum_{(i=p)}^{K(S \pm N)}\left\{\left(X_{p}\right)^{K}+\ldots\right\}^{K(Z \pm S \pm 1 \pm 1) / t} \\
& F(\cdot) G(\cdot)=\left\{\left(X_{p}\right)^{K}+\ldots\right\}^{K(Z \pm S \pm N \pm p) / t}=\rightarrow \sum_{(i=p)}^{K(S \pm N)}\left\{\left(X_{p}\right)^{K}+\ldots\right\}^{K(Z \pm S \pm 1 \pm 1) / t}
\end{aligned}
$$


In the formula: "= $\rightarrow$ " means normalization (the same below). $( \pm p=\rightarrow \pm 1)$ combination form of the order; $( \pm \mathrm{N}=\rightarrow \pm 1)$ combination form of calculus order; written repetition rate of cluster combination: $\mathrm{f}_{p}=K(\mathrm{~S} \pm N \pm p) \pm\{1\}$ 。(N-1);(p-1);

The above proof: eliminate the combined repetition rate $f_{p}$ to achieve normalization, called "harmonic analysis, linear expansion", also adapt to the cluster, calculus lifting order (derivative) $( \pm N)$ and element individual item order $( \pm P)$, they The corresponding combination coefficient $\left(1 / C_{(S \pm N \pm p)}\right)^{K}$ has a change in the form of a synchronized integer combination.

\section{Lanlangz Program Lemma and Round Logarithm}

\section{[Lemma] Reciprocal Theorem (B-H Conjecture)}

The reciprocity of the function was first proposed by Euler and Legendre. Gauss gave the first rigorous proof, called "number theory yeast", indicating that many mathematical function theorems are closely related to it. In 1967, Langlands proposed the "Basic Lemma" proposed by the Langlands program, called the reciprocal theorem ${ }^{[3]}$. In 1975, Beman-Hartmanis discovered a pair of reciprocal functions $\mathrm{G}\{\cdot\} \mathrm{F}\{\cdot\}$, called the B-H conjecture. The traditional proof is limited to "symmetry", and there is no objective "asymmetry" to show its incompleteness.

Here is a proof of the integrity of the reciprocity.

Prove that the various components of the function (element-factor) (group, algebraic geometric integer equation, circular logarithmic equation) have the reciprocity of integrity.

Assume: Countdown function (calculus) mean ( $\mathrm{K}=-1, \mathrm{~N}=-1, \mathrm{p}=-1$ )

Positive function (calculus) average $(\mathrm{K}=+1, \mathrm{~N}=+1, \mathrm{p}=+1)$;

$$
\begin{aligned}
& G_{0}(\cdot)=\left\{X_{0}\right\}^{K(Z \pm S-N-p)}=\left\{f X_{0}\right\}^{K(Z \pm S-N-p)}=\rightarrow\left\{X_{0}\right\}^{K(Z \pm S-N-1)} ; \\
& F_{0}(\cdot)=\left\{D_{0}\right\}^{K(Z \pm S+N+p)}=\left\{f X_{0}\right\}^{K(Z \pm S+N+p)}=\rightarrow\left\{D_{0}\right\}^{K(Z \pm S+N+1)} ;
\end{aligned}
$$

Combined repetition rate: $f_{( \pm N \pm p))}=(N-1)+(P-1)$;

where: : $\left\{f X_{a}\right\}^{ \pm 1}=f_{( \pm N \pm p))}$ indicates the number of repetitions in the combination of elements, and the elimination of the combined repetition rate is normalized.

[Certificate 1]: Reciprocity of functions: multi-element does not repeat the combination into sub-items, each sub-item combination is divided by

The set of combined coefficients should be the "characteristic modulus function" (average of positive, middle, and inverse functions) without losing the general Sex. Iterative method is adopted: multi-element combination (order value, item order) power $(Z / t)=K(Z \pm S \pm N \pm p) / t$ is sequentially increased or decreased.

$$
\begin{aligned}
\left.\left\{X_{0}\right\}^{K(Z \pm S \pm N \pm p)}=\sum_{(i= \pm N+p))}^{K(Z \pm S)}\left(1 / C_{(Z \pm S+p)}\right)^{ \pm 1}\left(x_{a} x_{b} \ldots\right)^{ \pm 1}+\ldots\right]^{ \pm(Z \pm S \pm N \pm p)} \\
=\left\{\sum_{(i= \pm N+p))}^{K(Z \pm S)}\left(1 / f C_{(Z \pm S \pm N+p}\right)^{ \pm 1}\left[\prod_{(i=p))}^{K(Z \pm S)}\left(f x_{a}\right)^{ \pm 1}+\ldots\right]^{ \pm 1}\right\}^{(Z \pm S \pm N \pm p)} \\
\left./ \sum_{(i=S)}\left(1 / f C_{(Z \pm S \pm N+p}\right)^{+1}\left[\left(f x_{a}\right)^{+1}+\left(f x_{b}\right)^{+1}+\ldots\right]^{ \pm 1}\right\}^{K(Z \pm S \pm N+p)} \\
\cdot\left\{\sum_{(i=S)}\left(1 / f C_{(Z \pm S \pm N+p}\right)^{+1}\left[\left(f x_{a}\right)^{+1}+\left(f x_{b}\right)^{+1}+\ldots\right]^{+1}\right\}^{K(Z \pm S \pm N+p)}
\end{aligned}
$$




$$
\begin{aligned}
& =\left\{\sum_{(i=S)}\left(1 / f C_{(Z \pm S \pm N+p}\right)^{-1}\left[\left(f x_{a}\right)^{-1}+\left(f x_{b}\right)^{-1}+\ldots\right]^{-1}\right\}^{K(Z \pm S \pm N-p)} \\
& \cdot\left\{\sum_{(i=S)}\left(1 / f C_{(Z \pm S \pm N+p}\right)^{+1}\left[\left(f x_{a}\right)^{+1}+\left(f x_{b}\right)^{+1}+\ldots\right]^{+1}\right\}^{K(Z \pm S \pm N+p)} \\
& =\left\{x_{0}\right\}^{K(Z \pm S \pm N-p)} \cdot\left\{x_{0}\right\}^{K(Z \pm S \pm N+p)}
\end{aligned}
$$

In the middle: $\quad\left\{\sum_{(i=s)}\left(1 / C_{(Z \pm S \pm N}\right)^{ \pm 1}\left[\prod_{(i=S)}\left(x_{a} x_{b} \ldots\right)^{ \pm 1}+\ldots\right]\right\}^{(Z \pm S \pm N)}$

$$
\begin{aligned}
& \left./ \sum_{(i=S)}\left(1 / f_{(Z \pm S \pm N+p}\right)^{+1} \sum_{(i=S)}\left(f_{x_{a}}{ }^{+1}+f_{X_{b}}{ }^{+1}+\cdots\right)^{+1}\right]^{K(Z \pm S \pm N+p)} \\
& =\left\{\sum_{(i=S)}\left(1 / f_{(Z \pm S \pm N+p}\right)^{-1} \sum_{(i=S)}\left(f_{a}{ }^{-1}+f_{x_{b}}{ }^{-1}+\cdots\right]\right\}^{K(Z \pm S \pm N-p)} \\
& =\left\{X_{0}\right\}^{K(Z \pm S \pm N-p)} ;
\end{aligned}
$$

\section{[Certificate 2] Reciprocal Theorem and Circular Logarithm}

Traditional mathematics introduces (symmetric) elliptic functions in reciprocity proofs. However, the elliptic function also has "symmetry and asymmetry".

Proof: Reciprocal Theorem and Circular Logarithm Relation

Let: reciprocal function or calculus equilibrium equation: independent variable $\{X)^{K(Z \pm S \pm N \pm p)}$ known (a priori, target, equilibrium) boundary condition $\left\{\mathrm{X}_{0}\right)^{\mathrm{K}(\mathrm{Z} \pm \mathrm{S}+\mathrm{N}+\mathrm{p})}$

certificate

$$
\begin{aligned}
& \left\{X_{\mathrm{o}}\right\}^{K(Z \pm S \pm N \pm p)}=\left\{X_{\mathrm{O}}\right)^{K(Z \pm S-N-p)}\left\{X_{\mathrm{O}}\right)^{K(Z \pm S+N+p)} \\
& =\left[\left\{X_{\mathrm{O}}\right)^{K(Z \pm S-N-p)} /\left\{X_{\mathrm{O}}\right)^{K(Z \pm S+N+p)}\right]\left\{X_{\mathrm{O}}\right)^{K(Z \pm S+N+p)}\left\{X_{\mathrm{O}}\right)^{K(Z \pm S+N+p)} \\
& =\left(1-\eta^{2}\right)^{K(Z \pm S \pm N \pm p)}\left\{X_{\mathrm{o}}\right\}^{K(Z \pm S+N+p)}\left\{X_{\mathrm{O}}\right\}^{K(Z \pm S+N+p)}
\end{aligned}
$$

Move a $\left\{X_{0}\right\}^{K(Z \pm S+N+p)}$ to the left of the equal sign

$$
\begin{gathered}
\left\{X_{0}\right\}^{K(Z \pm S \pm N \pm p)} /\left\{X_{0}\right\}^{K(Z \pm S+N+p)}=\{X)^{K(Z \pm S-N-p)} ; \\
\{X)^{K(Z \pm S \pm N \pm p)}=\left(1-\eta^{2}\right)^{K(Z \pm S \pm N \pm p)}\left\{X_{0}\right)^{K(Z \pm S \pm N \pm p)} ; \\
\left\{X_{0}\right)^{K(Z \pm S-N-p)}=\left(1-\eta^{2}\right)^{K(Z \pm S \pm N \pm p)}\left\{X_{0}\right\}^{K(Z \pm S+N+p)} ;
\end{gathered}
$$

\section{[Certificate 3] Round logarithmic normalization}

The infinite program topology of the logarithm can be normalized to the arithmetic of the linear factor (measurement, superposition).

Card: Quote [Certificate 1], [Certificate 2]:

$$
\begin{aligned}
& G_{0}(\cdot) F_{0}(\cdot)=\left\{X_{0}\right\}^{K(Z \pm S \pm N+p)}\left\{X_{0}\right\}^{K(Z \pm S \pm N-p)} \\
& =\sum\left(1 / C_{(S-p)}\right)^{ \pm 1} \prod_{(i=p)}\left[\left(x_{a} x_{\mathbf{b}} \ldots\right)^{-1}+\ldots\right]^{K(Z \pm S \pm N \pm p)} \\
& \left.=\sum_{(i=p)}\left(1 / C_{(S-p)}\right)^{ \pm 1} \sum_{(i=p)}\left[\left(x_{a}\right)^{ \pm 1}+\left(x_{b}\right)^{ \pm 1}+\ldots\right)+\ldots\right]^{K(Z \pm S-N \pm p)} \\
& =\sum_{(i=p)}\left(1 / f C_{(S-1)}\right)^{ \pm 1}\left[\sum_{(i=p)}\left(\left(f x_{a}\right)^{ \pm 1}+\left(f x_{b}\right)^{ \pm 1}+\ldots\right)+\ldots\right]^{K(Z \pm S-N \pm p)} \\
& \left.=\rightarrow\left(1 / C_{(S-1)}\right)^{ \pm 1} \sum_{(i=p)}^{(S \pm N)}\left[\left(x_{a}\right)^{ \pm 1}+\left(x_{b}\right)^{ \pm 1}+\ldots\right)\right]^{K(Z \pm S \pm N \pm p)} \\
& \left(1-\eta^{2}\right)^{\mathrm{K}(\mathrm{Z} \pm S \pm \mathrm{N} \pm \mathrm{P})}=\rightarrow\left(1-\eta^{2}\right)^{\mathrm{K}(\mathrm{Z} \pm \mathrm{S} \pm \mathrm{N} \pm 1)}
\end{aligned}
$$


Combined repetition ratefp; $f_{p}=K(S \pm N \pm p) \pm 1$; or $f_{p}=(N-1) \pm(p-1)$;

Where: $\left\{\mathrm{fx}_{\mathrm{a}}\right\}^{ \pm 1}$ indicates the number of repetitions ( $\mathrm{f}$ ) in the combination, and the element combination repetition rate $f$ is eliminated, which is normalized. Normalization demonstrates that each linear combination "monomer and overall element-factor" has a synchronous linear expansion (measurement, superposition).

\section{[Certificate4] Symmetry and asymmetry of reciprocal theorem}

The symmetry and asymmetry of the reciprocal theorem are reflected in the distance between the center point $\{X\}^{k(Z / t)}$ and the infinite points $\left\{\mathrm{X}_{\mathrm{a}}{ }^{\mathrm{k}}, \mathrm{Xb}_{\mathrm{b}}{ }^{\mathrm{k}}, \cdots \mathrm{X}_{\mathrm{p}}{ }^{\mathrm{k}}, \cdots\right\} \mathrm{k}(\mathrm{Z} / \mathrm{t})$ of the boundary line. symmetry.

(a), central elliptic function: closed elliptic curve, function $\left\{x_{a}{ }^{k}, x_{b}{ }^{k}, \cdots x_{p}{ }^{k}, \cdots\right\} k(Z / t)$, and two and two with the central equilibrium point $\{X\}^{k}(Z / t)$ The above (tangential) distance and the value of the point are symmetrical. It is called "central symmetric elliptical circle function" or "central symmetric circular function" for discrete calculation.

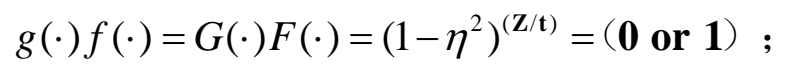

(b), eccentric elliptic function: closed asymmetric elliptic curve, function $\{x a k, x b k, \ldots x p k, \ldots\} k(z / t)$, to the tangent between the center point $\{\mathrm{X}\} \mathrm{k}(\mathrm{Z} / \mathrm{t})$ The distance and numerical points are asymmetrical, called "eccentric asymmetric elliptic function", and the entanglement calculation is performed.

$$
g(\cdot) f(\cdot)=G(\cdot) F(\cdot)=\left(1-\eta^{2}\right)^{(\mathbf{Z} / \mathbf{t})}=(\mathbf{0} \text { to } 1) ;
$$

In particular, the "asymmetry" of the circular logarithm has two states: the characteristic mode form (boundary) is invariant: the central function (equilibrium point) $\{\mathrm{X}\}^{\mathrm{k}(\mathrm{Z} / \mathrm{t})}$ position, numerical value change , and the boundary curve shape, the function $\left\{\mathrm{x}_{\mathrm{a}}{ }^{\mathrm{k}}, \mathrm{X}_{\mathrm{b}}{ }^{\mathrm{k}}, \cdots \mathrm{X}_{\mathrm{p}}{ }^{\mathrm{k}}, \cdots\right\} \mathrm{k}(\mathrm{Z} / \mathrm{t})$ changes, the change of the two is equivalent.

$$
\begin{gathered}
G(\cdot) F(\cdot)=\left(1-\eta^{2}\right)^{K(Z / t)} G_{0}(\cdot) \cdot F_{0}(\cdot) \\
=\left[\left(1-\eta^{2}\right)^{K(Z / t)} G_{0}(\cdot)\right] F_{0}(\cdot)=\left[\left(1-\eta^{2}\right)^{K(Z / t)} F_{0}(\cdot)\right] G_{0}(\cdot) ; \\
0 \leq \Sigma_{(i=Z \pm S \pm N)}\left(1-\eta^{2}\right)^{K(Z \pm S \pm N \pm p)} \leq 1 ;
\end{gathered}
$$

Among them: if compared with the "central symmetric circular function", then the "central symmetric elliptical circular function" also belongs to the "eccentric symmetry perfect circular function". For the circular logarithm of the circle with the perfect circle, the two will be additionally added. The logarithmic value between functions.

(c), The circular logarithm of linear and nonlinear functions

$$
\begin{aligned}
& G(\cdot) F(\cdot)=\rightarrow \sum_{(i=p)}^{(s \pm N)} \Pi_{(i=p)}^{(s \pm N)}\{g(\cdot) f(\cdot)\} \\
& =\rightarrow\left(1-\eta^{2}\right)^{K(Z \pm S \pm N \pm 2 \mathrm{P})}\left\{g_{0}(\cdot) f_{0}(\cdot)\right\} \\
& \left\{1 / C_{K(S \pm N \pm 2)}\right\}^{K(S \pm N \pm p) / t}=\left\{1 / C_{K(S \pm N \pm 1)}\right\}^{K(S \pm N \pm 2 p) / t} ;
\end{aligned}
$$


Where: the combination coefficient represents the square of the P combination of the elements in the multi-function, and the unit body element $\{\mathrm{g}(\bullet) \mathrm{f}(\bullet)\}$ has the duality of one-dimensional linearity and two-dimensional planarity. Physics is called wave-particle duality.

(d), normalized proof function (order value, item order) for integer expansion, analytical mapping to linear (arithmetic) calculation of circular logarithmic factor (called superposition, measure):

$$
\begin{aligned}
& \left(\eta^{2}\right)^{K(Z \pm S \pm N)}=\left(\eta^{2}\right)^{K(Z \pm S \pm N \pm 0)}+\left(\eta^{2}\right)^{K(Z \pm S \pm N \pm 1)}+\left(\eta^{2}\right)^{K(Z \pm S \pm N \pm p)}+\ldots ; \\
& \left(\eta^{2}\right)^{K(Z \pm S \pm N)}=\left(\eta_{1}^{2}\right)^{K(Z \pm S \pm N)}+\left(\eta_{2}\right)^{K(Z \pm S \pm N)}+\left(\eta_{p}\right)^{K(Z \pm S \pm N)}+\ldots ; \\
& (\eta)^{K(Z \pm S \pm N)}=\left(\eta_{1}\right)^{K(Z \pm S \pm N)}+\left(\eta_{2}\right)^{K(Z \pm S \pm N)}+\left(\eta_{p}\right)^{K(Z \pm S \pm N)}+\ldots ;
\end{aligned}
$$

[Certificate 5] The sum of the logarithm of the circle.

Eccentric elliptic functions (called self-defense functions) have become important mathematical research objects. Apply [Certificate 1]-[Certificate 4] reciprocal multiplication and continuous addition to the eigenmode function (average of positive, middle and inverse functions) to establish a symmetric and asymmetrical circular logarithmic equation whose factors are arithmetic Superimposed. The normalization proves that the sum of the logarithm of the circle is not substantially different from the product.

Proof is as follows:

$$
\text { Assume: } \begin{aligned}
\{X & \}^{K(Z \pm S \pm N \pm p)}=G(\cdot) F(\cdot) ;\{X\}^{K(Z \pm S \pm N \pm 1)}=g(\cdot) f(\cdot) ; \\
& \{X\}^{K(Z \pm S \pm N \pm p)}=\left(1-\eta^{2}\right)^{K(Z \pm S \pm N \pm p)}\{G(\cdot) F(\cdot)\}^{K(Z \pm S \pm N \pm p)} \\
& =\rightarrow \sum_{(i=p)}^{K(Z \pm S \pm N)} \Pi_{(i=p)}^{K(Z \pm S \pm N)}\left(1-\eta^{2}\right)^{K(Z \pm S \pm N \pm 1)}\left\{X_{0}\right\}^{K(Z \pm S \pm N \pm 1)} \\
& =\rightarrow \prod_{(i=p)}^{K(Z \pm S \pm N)} \sum_{(i=p)}^{K(Z \pm \pm \pm N)}\left(1-\eta^{2}\right)^{K(Z \pm S \pm N \pm 1)}\{g(\cdot) f(\cdot)\}^{K(Z \pm S \pm N \pm 1)} ;
\end{aligned}
$$

After formula (2.14) extracts the invariant eigenmode function, it leaves the linear expansion of the circular logarithm normalization, which becomes the arithmetic value of the factor to solve the function value.

$$
\begin{gathered}
\left(1-\eta^{2}\right)^{K(Z \pm S \pm N \pm p)}=\rightarrow \prod_{(i=p)}^{K(Z \pm S \pm N)}\left(1-\eta^{2}\right)^{K(Z \pm S \pm N \pm 1)} ; \\
\left(1-\eta^{2}\right)^{K(Z \pm S \pm N \pm p)}=\rightarrow \sum_{(i=p)}^{K(Z \pm S \pm N)}\left(1-\eta^{2}\right)^{K(Z \pm S \pm N \pm 1)} ; \\
0 \leq\left(1-\eta^{2}\right)^{K(Z \pm S \pm N)}=\prod_{(\mathrm{i}=\mathrm{Z})}\left(1-\eta^{2}\right)^{K(Z \pm S \pm N)}=\sum_{(\mathrm{i}=S)}\left(1-\eta^{2}\right)^{K(Z \pm S \pm N)} \leq 1 ;
\end{gathered}
$$

The formula (2.16) gives a unified description of the conjunction and the addition by the logarithm of the circle.

(1), $(k=+1)$ The multiplicative combination of multi-function elements is a negative (reverse) invariant eigenmode function.

(2), (k=-1) The combination of multiple function elements is a positive (positive) invariant eigenmode function. 
(3), $(k= \pm 0)$ The combination of multiple function elements is a neutral (zero) constant eigenmode function.

So far, [Certificate 1] [Certificate 5] has completely proved the reciprocal theorem: the combination of linear and nonlinear combinations of functions, and the existence of reciprocal "symmetry and asymmetry" becomes the mathematical basis of the basic theorem of circular logarithm series.

\section{The Circular Logarithm Theorem and Mathematical Conjecture}

The above [Literature] contains many mathematical conjectures, and becomes a circular logarithm theorem by proving whether these conjectures are or not.

[Theorem 1] The unit log logarithm, the first "1" specification invariance, called "Hodge conjecture"

Such as: Hodge conjecture: Is there any way to get the integer expansion of the function? That is, the function combination sub-item and the power function factor are synchronously changed, and a periodic "zero error" integer expansion is performed to realize a simple bonding of the geometric space.

Unit circle logarithm (called quantization) features: the sum is $\{0-1\}^{(\mathbf{Z} / \mathbf{t})}$ region, infinite elements (domain values, sets of numbers, point sets) various infinite combinations, each maintains each internal single The space, position, vector (referred to as plane, surface, space), numerical value, prime number distribution, event... of the body element belong to the feature mode function. After normalization, the solution is linearly expanded (measured, superimposed).

Definition: Unit log logarithm: "each sub-item of its own element $\sum\left\{\mathrm{x}_{\mathrm{h}}\right\}^{(\mathrm{Z} / \mathrm{t})}$ divided by the collection of its own elements unitary overall $\left\{\mathrm{X}_{\mathrm{H}}\right\}(\mathrm{Z} / \mathrm{t})$ :

$$
\begin{gathered}
\left(1-\eta_{H}{ }^{2}\right)^{(Z / t)}=\sum_{(i=p)}^{(Z \pm S \pm N)}\left(1-\eta_{h}{ }^{2}\right)^{(Z / t)}=\{1\}^{(Z / t)} ; \\
\left(1-\eta_{H}{ }^{2}\right)^{K(Z / t)}=\sum_{(i=Z)}\left[\left\{X_{h}\right\} /\left\{X_{H}\right\}\right]^{(\mathbf{Z} / \mathbf{t})} \\
=\sum_{(i=z)}^{K(Z \pm S \pm N \pm p)}\left(1-\eta_{h}{ }^{2}\right)^{K(Z / t)}=\{0 o r 1\}^{(\mathbf{Z} / \mathbf{t})} ; \\
\left(\eta_{H}{ }^{2}\right)^{K(\mathbf{Z} / \mathbf{t})}=\sum_{(i=z)}^{K(S \pm N)}\left(\eta_{h i}{ }^{2}\right)^{K(Z \pm S \pm N \pm p) / t} ; \\
\quad\left(\eta_{H}\right)^{k(\mathbf{Z} / \mathbf{t})}=\sum_{(i=z)}^{K(S \pm N)}\left(\eta_{h}\right)^{K(Z \pm S \pm N \pm p)} \\
\left\{x_{h}\right\}^{K(Z \pm S \pm N \pm 1)}=\left(\eta_{h}\right)^{K(Z \pm S \pm N \pm 1)} \cdot\left\{X_{0}\right\}^{K(Z \pm S \pm N \pm 1)} ;
\end{gathered}
$$

In particular, the distribution of the prime number theorem (PNT) is a "sparse and non-sparse" form, and "the prime number before a certain value is known" can be found in the logarithm of the unit circle.

[Theorem 2] The logarithm of the isomorphic circle, called the second "1" norm invariance, has a complete problem called "P=NP"

Such as: "P = NP complete problem": It is required to prove that "simple polynomial and complex polynomial have isomorphic time calculation", if the proof is established as the second "1" norm invariance theorem. 
Define the logarithm of the isomorphic circle: "The average of the set of individual elements $\sum\left\{\mathrm{x}_{\mathrm{oh}}\right\}^{(\mathrm{z} / \mathrm{t})}$ divided by the total mean of its own elements

$\sum\left\{\mathrm{X}_{\mathrm{OH}}\right\}^{(\mathrm{z} / \mathrm{t})}$, with isomorphism $\left(1-\eta_{\mathrm{H}}{ }^{2}\right)^{(\mathrm{z} / \mathrm{t})}=\{0 \text { to } 1\}^{(\mathrm{z} / \mathrm{t})}$ " . Feature: Each component of the function has the same logarithmic form, providing a logarithmic set with the dimensionless quantum function as the base, called the logarithm of the isomorphic circle.

Assume: $\left.\quad\left\{X_{0}\right)^{K(Z \pm S \pm N \pm 1)}=B / C_{(S \pm N \pm 1)} ; X_{0}\right\}^{K(Z \pm S \pm N \pm 1)}=\left\{{ }^{K(S \pm N)} \sqrt{ } X\right\}^{K(Z \pm S \pm N \pm 1)}$

Introduce [Certificate 1]-[Certificate 5], normalization $\left\{X_{0}\right)^{(\mathbf{Z} / \mathbf{t})}=\rightarrow\left\{X_{0}^{ \pm 1}\right)^{K(Z \pm S \pm N \pm 1)}$

Assume:

$$
\begin{aligned}
& \left(1-\eta^{2}\right)^{(\mathbf{Z} / \mathbf{t})}=\left[\left\{x^{-1}\right\} /\left\{x_{0}\right\}\right]^{K(Z \pm S \pm N \pm p)}=\sum_{(i=p)}^{K(Z \pm S \pm N)}\left[^{K(S \pm N)} \sqrt{ }\{X\} /\left\{X_{0}\right\}\right]^{(\mathbf{Z} / \mathbf{t})} ; \\
& A x^{K(Z \pm S \pm N \pm 0) / t}+B x^{K(Z \pm S \pm N \pm 1) / t}+\ldots+P x^{K(Z \pm S \pm N \pm p) / t}+\ldots \\
& =C_{(S \pm N \pm 0)} X^{K(Z \pm S \pm N \pm 0) / t}+C_{(S \pm N \pm 1)} X^{K(Z \pm S \pm N \pm 1) / t}+\ldots+C_{(S \pm N \pm p)} X^{K(Z \pm S- \pm N \pm p) / t}+\ldots \\
& =\left(1-\eta^{2}\right)^{(\mathbf{Z} / \mathrm{t})}\left\{X_{0}\right\}^{(\mathbf{Z} / \mathbf{t})} \text {; } \\
& \left(1-\eta_{T}{ }^{2}\right)^{(\mathrm{Z} / \mathrm{t})}=\sum_{(i=p)}^{K(S \pm N)}\left[\left\{X_{\mathrm{O}}{ }^{-1}\right\} /\left\{X_{\mathrm{o}}{ }^{+1}\right\}\right]^{(\mathrm{Z} / \mathrm{t})} \\
& =\sum_{(i=p)}^{K(S \pm N)}\left[\left\{\left\{^{(S \pm N)} \sqrt{ } X\right\} /\left\{X_{\mathrm{O}}{ }^{+1}\right\}\right]^{(\mathrm{Z} / \mathrm{t})}\right. \\
& =\left\{\begin{array}{lll}
\mathrm{O} & \text { to } & \}^{(\mathrm{z} / \mathrm{t})}
\end{array}\right.
\end{aligned}
$$

The isomorphism of the isomorphic logarithm is normalized to a linear combination.

$$
\begin{aligned}
\left(1-\eta_{T}{ }^{2}\right)^{(\mathrm{Z} / \mathrm{t})}= & \rightarrow\left(1-\eta_{0}{ }^{2}\right)^{(\mathrm{Z} / \mathrm{t})}=\rightarrow\left(1-\eta_{1}\right)^{(\mathrm{Z} / \mathrm{t})}=\rightarrow \ldots\left(1-\eta_{p}{ }^{2}\right)^{(\mathrm{Z} / \mathrm{t})} \ldots ; \\
& 0 \leq\left(1-\eta_{T}{ }^{2}\right)^{(\mathrm{Z} / \mathrm{t})} \leq\{1\}^{(\mathrm{Z} / \mathbf{t})} ;
\end{aligned}
$$

The invariant features of the isomorphic circular logarithms in the unit body $\{1\}(z / t)$ have consistent isomorphic topologies, so that they have the same topological rules and the periodicity of the logarithm of the circle. Implement equivalent substitution between elements. In this way, each element in the complex gauge field has a unified topological rule, and the calculation is purified and unified.

[Theorem 3] Relatively symmetric circular logarithm, called the third "1" norm invariance, there is a problem of symmetric conservation

The holistic state of the mean value theorem of traditional calculus in its domain, including Rolle's theorem, Lagrangian theorem, Cauchy's theorem, and Taylor's theorem, becomes a bridge between the relationship between the derivative value and the function value. The local nature of the 'derivative' is a tool that approximates the overall properties of the function. The fact is: under the condition of relativity, each ratio has its own value. A derivative point does not reflect the symmetry and asymmetry of the function as a whole, and establishes the connection between the local point and the whole function through the concept of symmetric circular logarithm.

Define the relative symmetric circular logarithm: the element total term (whole) function $\{X\}$ divided by the element's own term (individual) mean $\{X 0\}$, and obtain the reciprocal symmetric logarithm (1- $\left.\eta_{\mathrm{k}}{ }^{2}\right)^{(\mathrm{Z} / \mathrm{t})}$. Features: According to the function has symmetry and asymmetry, the logarithm of the unit circle and the logarithm of the isomorphic circle are converted into peri 
There are: (1), odd-numbered circular logarithm:

$$
\begin{aligned}
& \left(1-\eta_{k}{ }^{2}\right)^{(Z / t)}=\left[\left\{X_{H}\right\} /\left\{X_{0}\right\}\right]^{(Z / t)} \\
= & \sum_{(i=A)}\left(1-\eta_{k}^{2}\right)^{+(Z / t)}+\sum_{(i=B)}\left(1-\eta_{k}{ }^{2}\right)^{-(Z / t)}+\sum_{(i=C)}\left(1-\eta_{k}{ }^{2}\right)^{0(Z / t)} \\
= & \left(1-\eta_{A}{ }^{2}\right)^{+(Z / t)}+\left(1-\eta_{B}{ }^{2}\right)^{-(Z / t)}+\left(1-\eta_{C}{ }^{2}\right)^{0(Z / t)} \\
= & {[0 t o 1] }
\end{aligned}
$$

(2) Even-numbered circular logarithm:

$$
\begin{aligned}
& \left(1-\eta_{k}{ }^{2}\right)^{(Z / t)}=\left[\left\{X_{H}\right\} /\left\{X_{0}\right\}\right]^{(Z / t)} \\
= & \sum_{(i=A)}\left(1-\eta_{k}{ }^{2}\right)^{+(Z / t)}+\sum_{(i=B)}\left(1-\eta_{k}{ }^{2}\right)^{-(Z / t)} \\
= & \left(1-\eta_{A}{ }^{2}\right)^{+(Z / t)}+\left(1-\eta_{B}{ }^{2}\right)^{-(Z / t)} \\
= & {[0 t o 1] }
\end{aligned}
$$

(3) Relative symmetry circle logarithm factor

$$
\left|\sum\left(+\eta_{A}\right)\right|^{(\mathbf{Z} / \mathbf{t})}=\left|\sum\left(-\eta_{B}\right)\right|^{(\mathbf{Z} / \mathbf{t})}=\left\{\left[\left\{X_{A}\right\}-\left\{X_{B}\right\}\right] /\left[\left\{X_{A}\right\}+\left\{X_{B}\right\}\right]\right\}^{(\mathbf{Z} / \mathbf{t})} ;
$$

Symmetric circle logarithmic proof results:

(1) Converting the asymmetry problem into a relative symmetry problem by circular logarithmic factor processing. The unity of symmetry and asymmetry of the reciprocal theorem is solved.

(2), the logarithmic equation and the eigenmode function (prime, integer, arbitrary value), there are two structures of the even function (prime, integer) and singular function (prime, integer).

(a) When ( $\left.\mathrm{P}=\mathrm{P}_{1}+\mathrm{P}_{2}\right)$ two prime numbers and integer combinations get the smallest "evenness function."

(b) When ( $\left.\mathrm{P}=\mathrm{P}_{1}+\mathrm{P}_{2}+\mathrm{P}_{3}\right)$ three prime numbers and integer combinations get the smallest "singularity function".

[Theorem 4], the principle of circular logarithmic zero (limit, phase transition, center point)

Defining the zero point (limit, phase change, center point): According to the principle of relativity, the ratio of any two uncertain values has a certain symmetrical value as the center point between " 0 to 1 " of the unrelated mathematical model. The relative symmetry of the reciprocal relationship between the left and right sides is called the zero point. Proof is as follows:

$$
\text { because: } \quad \begin{aligned}
\left(1-\eta^{2}\right)^{(Z / t)}= & \prod\left(1-\eta^{2}\right)^{(Z / t)}=\sum\left(1-\eta^{2}\right)^{(Z / t)}=\{0,1\}^{(Z / t)} ; \\
& \left(1-\eta^{2}\right)^{+(Z / t)}+\left(1-\eta^{2}\right)^{-(Z / t)}=1 ; \\
& \left(1-\eta^{2}\right)^{+(Z / t)} *\left(1-\eta^{2}\right)^{-(Z / t)}=1 ;
\end{aligned}
$$

Solution (7.1) simultaneous equation

$$
\left|\left(1-\eta^{2}\right) \sim(\eta)\right|^{K(Z / t)}=(0,1 / 2,1)^{K(Z / t)} ; \quad(K=+1,0,-1) ;
$$

Equation (7.2) has a stable logarithmic zero (limit, critical, bound) 
In the circle (ball) coordinates,

There are: $\mathrm{S}=\mathrm{R} \theta$ (two-dimensional plane circle) circular logarithm representation $\left(1-\eta^{2}(r, \theta, x, y, z)\right)$

$F=R^{2}(\theta \phi) \quad$ (two-dimensional spherical surface circle) Circular logarithm representation(1- $\left.\eta^{2}(r, \phi, \theta, x, y, z)\right)$ $F=R^{2}(\theta \phi)$

Cartesian coordinate system factor

$$
\eta_{(\mathbf{x}, \mathbf{y}, \mathbf{z})}=[0,1 / 2,1,2]^{K(\mathrm{Z} / t)} ;
$$

Circle (sphere)) zero factor

$$
\eta_{(r, \varphi, \theta)}=\eta_{(r \wedge, \varphi, \theta)}=\left[0, \theta_{0} \pm(\pi / 4,2 \pi / 4,3 / 4, \pi, 2 \pi)\right]^{K(Z / t)}
$$

The zero theorem ensures the stability and smoothness of the non-normal zero of the infinitely combined elements of the Riemannian function.

Among them:

(1), for the Riemann (zero point) conjecture: the sum of the reciprocal of the Riemann function and then reciprocal, the average value of the reciprocal function is not lost, and is at the abnormal zero point on the critical line (parity function structure) $\{1 / 2\}^{+K(Z / t)}$ : That is: the non-normal zero of the Riemannian function (prime, integer) is at the center of symmetry.

(2), for Goldbach (zero) conjecture $\{1 / 2\}^{-K(Z / t)}=\left.\{2\}\right|^{-K(Z / t)}$ even function structure: two singular functions (prime) An abnormal zero that exists on the critical line.

\section{[Theorem 5], parallel/serial circular logarithm theorem}

The function-circle logarithmic change rule has nothing to do with the choice of coordinates. The origin can be set to the value of the change between the start and end points anywhere. There are many other forms of circular logarithmic equations (algebraic clusters):

Defining parallel/serial equations: calculus dynamics equations: There are parallel/serial symmetry and asymmetry equations with various levels of multi-level elements and parameters.

Multi-level calculus dynamic equation consisting of parallel/serial: its power function:

heve: $\quad(Z / t)=K(Z \pm S \pm H \pm N \pm p) / t ; \quad H=H_{A}+H_{B}+\ldots+H_{P} \ldots ;$

(1) The serial equation (continuous multiplication combination) is the composite hierarchy of $H$, "reciprocal function construction"

$$
\begin{aligned}
& \left\{X_{0 H}\right\}^{(Z / t)}=\left\{\left(^{(S \pm H \pm N)} \sqrt{ } \prod_{(i=H)}\left(X_{H p}\right)\right\}^{(Z / t)}\right. \\
& =\prod_{(i=H)}\left(1 / C_{(S-H \pm N \pm p)}\right)\left\{X_{A} \cdot X_{B} \cdot \ldots X_{P} \cdot \ldots\right\}^{K(S \pm H \pm N \pm p) / t} \\
& =\left(1 / f C_{(S-H \pm N \pm p)}\right)^{-1}\left\{\left(f X_{A}\right)^{-1}+\left(f X_{B}\right)^{-1}+\ldots+\left(f X_{P}\right)^{-1}+\ldots\right\}^{\mathbf{K}(S-H \pm N \pm p) / t} \\
& =\sum_{(i=p)}^{K(S-H \pm N)}(1 / H)^{-1}\left\{\left(X_{A}\right)^{-1}+\left(X_{B}\right)^{-1}+\ldots+\left(X_{P}\right)^{-1}+\ldots\right\}^{\mathbf{K}(S-H \pm N \pm 1) / t} ;
\end{aligned}
$$

(2) Parallel equations (continuously combined) are H's compound hierarchy "positive function construct" 


$$
\begin{aligned}
& \left\{X_{0 H}\right\}^{(Z / t)}=\left\{\left(^{(S \pm H \pm N)} \sqrt{ } \sum_{(i=H)}\left(X_{H P}\right)\right\}^{(Z / t)}\right. \\
& =\sum_{(i=H)}\left(1 / C_{(S+H \pm N \pm p)}\right)\left\{X_{A}+X_{B}+\ldots+X_{P}+\ldots\right\}^{K(S \pm H \pm N \pm p) / t} \\
& =\left(1 / C_{(S+H \pm N \pm p)}\right)^{-1}\left\{\left(f X_{A}\right)^{+1}+\left(f X_{B}\right)^{+1}+\ldots+\left(f X_{P}\right)^{+1}+\ldots\right\}^{K(S+H \pm N \pm p) / t} \\
& =\sum_{(i=p)}^{K(S+H \pm N \pm p)}(1 / H)^{+1}\left\{\left(X_{A}\right)^{+1}+\left(X_{B}\right)^{+1}+\ldots+\left(X_{P}\right)^{+1}+\ldots\right\}^{K(S-H-N-1) / t}
\end{aligned}
$$

(3) Tthree-dimensional coordinates:

Taking the center point of any balance as the coordinate origin, introducing three-dimensional rectangular coordinates $(\boldsymbol{I}, \boldsymbol{j}, \boldsymbol{k})$, the sphere inside (outside Can be mapped to the coordinate axis,

$$
\left(1-\eta^{2}\right)^{K(z / t)}=\left(1-\eta_{x}^{2}\right)^{K(z / t)} \mathbf{i}+\left(1-\eta_{y}^{2}\right)^{K(z / t)} \mathbf{j}+\left(1-\eta_{z}^{2}\right)^{K(z / t)} \mathbf{k} ;
$$

(4),Tthree-dimensional spherical coordinates

Taking the center point of any balance as the coordinate origin, the three-dimensional right-angle spherical coordinate [xy] is introduced corresponding to the normal axis $k$; [yz] corresponds to the normal axis $\mathrm{i}$; [zx] corresponds to the normal axis j), and the sphere (outside) can be Map to the coordinate axis.

$$
\left(1-\eta_{[u v]}^{2}\right)^{K(z / t)}=\left(1-\eta_{[y z]}\right)^{K(z / t)} \boldsymbol{i}+\left(1-\eta_{[z x]}^{2}\right)^{K(z / t)} \boldsymbol{j}+\left(1-\eta_{[x y]}\right)^{K(z / t)} \boldsymbol{k} ;
$$

where: $\left(1-\eta^{2}\right)^{K(Z \pm S \pm[A+B+P+\ldots] \pm N) / t},\left(1-\eta_{[u v]}\right)^{K(Z / t)}\left(1-\eta_{[u v]}^{2}\right)^{K(Z / t)}$ has its own level of

polynomial and micro The serial parallel expansion of the integrals.

among them: $\left(1-\eta^{2}\right)^{\mathrm{K}(Z \pm S \pm[A+B+P+\ldots] \pm N) / t},\left(1-\eta_{[u v]}\right)^{K}(Z / t) ;$

(5), The logarithmic factor expansion

$$
\begin{aligned}
& \left(1-\eta^{2}\right)^{(Z / t)}=\left(1-\eta^{2}\right)^{K(Z \pm[A+B+P+\ldots]) / t} \\
& =\left(1-\eta_{A}{ }^{2}\right)^{K(Z \pm A) / t}+\left(1-\eta_{B}{ }^{2}\right)^{K(Z \pm B) / t}+\ldots+\left(1-\eta_{P}{ }^{2}\right)^{K(Z \pm P) / t}+\ldots ; \\
& (\eta)^{(Z / t)}=\left(\eta_{A}\right)^{(Z / t)}+\left(\eta_{B}\right)^{(Z / t)}+\ldots+\left(\eta_{P}\right)^{(Z / t)}+\ldots ; \\
& \left(\eta^{2}\right)^{(Z / t)}=\left(\eta_{A}{ }^{2}\right)^{(Z / t)}+\left(\eta_{B}{ }^{2}\right)^{(Z / t)}+\ldots+\left(\eta_{P}{ }^{2}\right)^{(Z / t)}+\ldots+;
\end{aligned}
$$

Equations (8.1)-(8.7) are based on random and regular decomposition and synthesis of multi-level unit bodies based on arbitrary integer functions. They become parallel/serial polynomial equations and can be resolved into parallel/serial "three" The high power circular logarithm equation consisting of 1 'norm invariance' (unit log logarithm, isomorphic log logarithm, relative symmetric circle logarithm) is subjected to linear and nonlinear arithmetic calculations (superposition). Meet the blockchain, quantum computing, and take into account the requirements of "security, openness, privacy, fairness, and borderline". The feature module function is mastered by the key content, and the private content cannot be cracked and will not be disclosed.

[Theorem 6], equivalent replacement principle (covariation principle)

For the principle of equivalent substitution between the multi-element ( $\cdots$ uv $\cdots)$ and the controllable (a priori value) $\left\{U_{0}\right\}\left\{\bigvee_{0}\right\}$, it is proved as follows.

there are: Traditional partial differential equations, which are differential equations of two-two combinations of multi-element $(\mathrm{S} \pm \mathrm{N})$ orders. Its traditional points. The variable method: $\mathrm{d}(\cdots \mathrm{uv} \cdots)$ 
becomes the general solution to the partial differential dynamics equation: any $S$ unknown elements of the $\mathrm{P}$ combination of higher-order $\mathrm{N}$-order partial differential equations of $\mathrm{S}$ multielement $\{\ldots u, v \ldots\}^{\mathrm{K}(\mathrm{S} \pm \mathrm{N} \pm \mathrm{p}) / \mathrm{t}}( \pm \mathrm{N}=\mathrm{N})$,

here: $\quad d^{(S \pm 2 \pm 2)}(\ldots u v \ldots)=d^{(S \pm 2 \pm 2)}\{\ldots+v d u+u d v+\ldots\}$;

or:

$$
\begin{aligned}
& \partial^{(S \pm N \pm p)}\left[\ldots\left(\prod_{(i=p)}(u v \ldots)\right]=\partial^{(S \pm N \pm p)}\{\ldots+v \partial(u \ldots)+u \partial(v \ldots)+\ldots\} ;\right. \\
& \{X\}^{\mathbf{K}(S \pm N \pm p) / t}=\prod_{(i=p)}\{u v \ldots\}^{\mathbf{K}(S \pm N \pm p) / t} 。
\end{aligned}
$$

Solve: The $\{u, v\}$ value of any two unknown elements of the $\mathrm{P}$ combination in the second-order partial differential dynamics equation of $\mathrm{S}$ multi-element.

Traditional mathematical solutions are very difficult.

Proof: Select the three order of the known calculus equation coefficients $A, B, C$ and the boundary

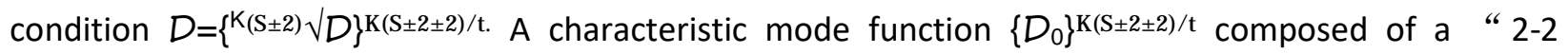
combination" of the second order $(\mathrm{P}=2)$ of the $(\mathrm{S})$ element.

(1) The relationship between the eigenmode function $\left\{D_{0}\right\}^{\mathrm{K}(\mathrm{S} \pm 2 \pm 2) / \mathrm{t}}$ and the polynomial coefficients and combination coefficients:

$$
,\left\{D_{0}\right\}^{K(S \pm N \pm 0) / t}=\mathbf{A} / C_{(S \pm N \pm 1)}\left\{D_{0}\right\}^{K(S \pm N \pm 1) / t}=\mathbf{B} / C_{(S \pm N \pm 2)}\left\{D_{0}\right\}^{K(S \pm N \pm 0) / t}=\mathbf{C} / C_{(S \pm N \pm p)}
$$

make:

$$
\{X\}^{\mathbf{K}(S \pm 2 \pm p) / t}=\left\{\ldots+C \prod_{(i=2)}(u v)+\ldots\right\}^{K(S \pm 2 \pm p) / t}=\left\{{ }^{K(S \pm 2)} \sqrt{ } D\right\}^{K(S \pm 2 \pm p) / t}
$$

(2) Normalized isomorphic unit circle logarithm (H=S)

$$
\begin{aligned}
& \left(1-\eta_{H}^{2}\right)^{K(S \pm 2 \pm p) / t}=\left\{{ }^{K(S \pm N)} \sqrt{ } / D_{0}\right\}^{K(S \pm N \pm p) / t}=\sum_{(i=S)}\left\{^{K(S \pm N)} \sqrt{ } D / D_{0}\right\}^{K(S \pm N \pm 1) / t}=1 ; \\
& \left\{D_{0}\right\}^{\mathbf{K}(S \pm 2 \pm 2) / t}=\left\{C / C_{(S \pm 2 \pm 2)}\right\}^{K(S \pm 2 \pm 2) / t} \\
& =\Sigma_{(i=S+2+2)}\left(1 / C_{(S \pm 2 \pm 2}\right)\left\{\prod_{(i=2)}(u v)+\ldots\right\}^{K(S \pm 2 \pm 2) / t} \\
& =\left(1 / f_{p} C_{(S \pm 2 \pm 2)}\right)\left\{\Sigma_{(i=S)}(1 / S)\left(f_{p} u+f_{p} v \ldots\right)+\right\}^{K(S \pm 2 \pm 2) / t} \\
& =\rightarrow\left\{B / C_{(S \pm N \pm 1)}\right\}^{\mathbf{K}(S \pm N \pm 1) / t} \\
& =\rightarrow\left(1 / C_{(S \pm N \pm 1)}\right) \Sigma_{(i=S)}\left\{\left(u^{+1}+v^{+1}\right)+\ldots\right\}^{\mathbf{K}(S \pm N \pm 1) / t} \\
& =\rightarrow \Sigma_{(i=S)}\left\{\ldots+\left(U_{0}+V_{0}\right) \ldots\right\}^{\mathbf{K}(S \pm N \pm 1) / t} ;
\end{aligned}
$$

(3) Solving and Equivalent Permutation of Arbitrary Higher Order Differential Equations

$$
\begin{aligned}
& \{u\}^{\mathrm{K}(S \pm N \pm p) / t}=\rightarrow\left(1-\eta_{u}{ }^{2}\right)^{\mathrm{K}(S \pm N \pm 1) / t}\left\{U_{0}\right\}^{\mathrm{K}(S \pm N \pm 1) / t} ; \\
& \{v\}^{\mathrm{K}(S \pm N \pm p) / t}=\rightarrow\left(1-\eta_{v}{ }^{\mathrm{K}(S \pm N \pm 1) / t}\left\{V_{0}\right\}^{\mathrm{K}(S \pm N \pm 1) / t} ;\right. \\
& \{u v \ldots\}^{\mathrm{K}(S \pm N \pm p) / t}=\left(1-\eta^{2}\right)^{\mathrm{K}(S \pm N \pm p) / t} \cdot\left\{U_{0} V_{0} \ldots\right\}^{K(S \pm N \pm p) / t} ; \\
& \mathrm{t}=\left(1-\eta_{\mathrm{e}}{ }^{2}\right)^{\mathrm{K}(Z \pm S \pm N \pm p)} \mathrm{t}_{0} ;
\end{aligned}
$$


Equations (9.1)-(9.5) show that all the log-log factor change rules are consistent after the function element is converted to the eigenmode function (mean function) in the entangled state.

It is found that any one (or more than two) elements in the function are consistent with the change of the logarithmic factor of the circle, and can be equivalently substituted (called the covariation principle). That is to say, knowing one or two entangled elements of a cluster, the equivalent replacement principle can be applied to solve the whole group element, and finding any one of the elements-factors has an equivalent solution.

[Theorem 7], the stability, optimization, evaluation and weighted average of the logarithm of the circle

The statistical learning theory should be statistical entanglement and discrete calculation. For each data, the data is summarized intoH=[A+B+...P+...], and the power function of the logarithm is written $(\mathrm{Z} / \mathrm{t})=\mathrm{K}(\mathrm{Z} \pm[\mathrm{A}+\mathrm{B}+\mathrm{P}+\ldots]) / \mathrm{t}$.

$$
\begin{aligned}
& \left(1-\eta^{2}\right)^{(Z \pm H] / t)}=\left(1-\eta^{2}\right)^{K(Z \pm[A+B+P+\ldots]) / t} \\
& =\sum_{(i=H \pm N \pm P)}^{K(Z \pm H)}\left(1-\eta_{p}{ }^{2}\right)^{K(Z \pm H) / t} ; \\
& \left(\eta_{(S \pm H)}\right)^{(Z / t)}=\left(\eta_{A}\right)^{(Z / t)}+\left(\eta_{B}\right)^{(Z / t)}+\ldots+\left(\eta_{P}\right)^{(Z / t)}+\ldots ; \\
& \left.\left(\eta_{(S \pm H)}\right){ }^{(Z / t)}=\left(1 / f C_{(S \pm H)}\right)\right)\left[\left(f \eta_{A}\right)^{(Z / t)}+\left(f \eta_{B}\right)^{(Z / t)}+\ldots+\left(f \eta_{P}\right)^{(Z / t)}+\ldots\right] \\
& =\rightarrow\left(1 / C_{(S \pm H \pm 1)}\right)^{K}\left[\left(\eta_{A(S \pm H)}\right)^{(Z / t)}+\left(\eta_{B(S \pm H)}\right)^{(Z / t)}+\ldots+\left(\eta_{P(S \pm H)}\right)^{(Z / t)}+\ldots\right] \\
& =(0 \text { or } 1) ;
\end{aligned}
$$

Obtained:

(1), stability, error analysis, machine learning: In the discrete analysis, the judgment tends to $\{0\}$ (z/t) as the maximum stability.

$$
\begin{aligned}
& \left(1-\eta_{(S \pm H)}{ }^{2}\right)=\left\{\left[\sum_{(S \pm H)}\left(\eta_{A(S \pm H)}^{2}\right)-\sum_{(S \pm H)}\left(\eta_{B(S \pm H)}{ }^{2}\right)\right] /\left[\sum_{(S \pm H)}\left(\eta_{A(S \pm H)}^{2}\right)-\sum_{(S \pm H)}\left(\eta_{B(S \pm H)}{ }^{2}\right)\right]\right\}^{(Z / t)} \\
& =\{0\}^{(Z / t)}
\end{aligned}
$$

(2) Optimum, extreme value, weighted average: In mathematical analysis, the judgment tends to $\{0,1 / 2,1\}$ (z/t) as the maximum optimization.

$$
\begin{aligned}
& \left(1-\eta_{(S \pm H)}{ }^{2}\right)=\left\{\left[\sum_{(S \pm H)}\left(\eta_{A(S \pm H)}^{2}\right)-\sum_{(S \pm H)}\left(\eta_{B(S \pm H)}^{2}\right)\right] /\left[\sum_{(S \pm H)}\left(\eta_{A(S \pm H)}{ }^{2}\right)-\sum_{(S \pm H)}\left(\eta_{B(S \pm H)}{ }^{2}\right)\right]\right\}^{(Z / t)} \\
& \approx\{0,1 / 2,1\}^{(Z / t)}
\end{aligned}
$$

(3) There are two states of probability calculation: discrete type and entangled zero point and zero boundary line:

Discrete analysis: based on the symmetry of the forward and reverse lines, with a linear line as the critical line.

Entangled analysis: based on the asymmetry (ie, relative symmetry) of the positive and negative curves, the circular logarithm of the quadratic curve is the critical line. 


\section{Unification of Circular Logarithm and Mathematical Formula}

\subsection{Contemporary mathematics needs to be transformed}

Mathematical combination of multi-element multiplication for arbitrary functions can be a calculus polynomial. The first encounter is that traditional calculus and group logic algebra are the mainstream of current mathematical algorithms and need to be modified.

(a), differential: is the slope of the function image at a certain point, that is, the ratio of the ordinate increment $(\Delta y)$ and the abscissa increment $(\Delta x)$ at $\Delta x \rightarrow 0$. Generally expressed as $\mathrm{df}(\mathrm{x}) / \mathrm{dx}$. It belongs to infinity and is smaller than infinity.

(b), Integral: is the sum of the product $f(x)$ and the infinitesimal small product, generally expressed as $\int{ }^{n} f(x) d x$.

Traditional calculus introduces the "limit" toward the value of "zero point or a fixed value", and the axiomatized self/self is equal to "1" and enters the discrete calculation.

However, the infinity of the principle of relativity is not necessarily "0 or 1 " than infinity. It is unclear to show that the concept of infinitesimal limits. The fact is that "the combination of multi-element multiplication can be discretely calculated 'Oor1' and the entangled type '0to1'. For the combination of multi-element multiplication with interaction entanglement, there is nothing to do, and the infinitesimal discrete calculation is used to replace the entanglement. The calculation reflects the defects of traditional mathematics. The key of the defect is that the "known boundary conditions and the two coefficients of unknown variables" which are different between the discrete type and the entangled type cannot be treated differently. The round logarithm is clearly and clearly distinguished. The difference and unity of the species, through the transformation of calculus symbols, the establishment of a unified calculus equation, converted into a unified circular logarithmic equation and eigenmode function form. This is a calculus function written for the combined set of multi-element multiplication, to be precise Unified description and calculation are possible. Principles and methods are as described above.

\subsection{The Connection Between Traditional Functions and Circular Logarithms}

The real number is a function of the independent variable. The complex variable function eliminates the "complex $i "$ content due to the existence of the two equilibrium calculation results of the equation $\{0,2\}$, and unifies the concept of "function".

when: number theory is a function that studies the infinite elements (integer, prime, fraction, point set) into arbitrary integers and prime numbers, and analyzes the mapping to the logarithmic equation to obtain arbitrary integer or prime numbers, which solves the algebraic integer problem. The realization of the function and algorithm by the logarithm of the circle is unified. (Limited to a limited number of cases).

\section{(1) Riemann's function}

Riemann sees $\zeta(S)$ as a function of complex variable(s) and extends it to a meromorphic function over the entire complex plane. Become $\zeta(\mathrm{S}), \zeta(\mathrm{S})=\left\{\sum \mathrm{n}^{-\mathrm{S}}\right\}^{+1}$ "the sum of the reciprocals" , now implement "the sum of the reciprocal numbers" and become the "average of the reciprocal function" , written as: $\zeta(S)=\left\{\sum n^{-S}\right\}^{-1}, \zeta(S)=\left\{\sum n^{K}\right\} ; K=(+1,0,-1)$ controls the convergence of the $\zeta$ function without loss of generality. 
If the logarithm of the circle is normalized, it is the "linear eigenmode function", which is related to the harmonic analysis.

$$
\zeta(S)^{(Z / t)}=\left\{\sum n^{+S}\right\}^{+(Z / t)}\left\{\sum n^{-S}\right\}^{-(Z / t)}=\left(1-\eta^{2}\right)^{(Z / t)} \zeta\left(S_{0}\right)^{(Z / t)}
$$

\section{(2), prime number theorem (PNT):}

For example, the famous "Plastic Number Theorem (PNT)" is a $(x / \operatorname{lnx})$ describing the irregular sparse distribution of prime numbers, and $\mathrm{O}\left(\mathrm{X}^{(1 / 2)+\varepsilon}\right)$ is obtained. This is based on the fixed value (with constant e or 10$)$ as the base, and the "residue $\varepsilon$ " cannot be eliminated, and the prime and integer solutions are not obtained.

The unit log logarithm allows the internal factors to be unevenly distributed, and maintains the elements of the prime elements in each unit of the unit log logarithm, without affecting the prime and number solutions of $\mathrm{O}\left(\mathrm{X}^{(1 / 2)+0}\right)$ The realization of the number of prime numbers without the "residue $\varepsilon$ " unit body is realized.

$$
\begin{aligned}
& \left(1-{\eta_{H}}^{2}\right)^{(Z / t)}=\sum_{(i=p)}(Z / t) \zeta\left(S_{h}\right) / \zeta\left(S_{H}\right)=1 ; \\
& O\left(X^{(1 / 2)+\varepsilon}\right) \rightarrow O\left(X^{(1 / 2)+\mathbf{0}}\right), \\
& \sum_{(i=S)}(x / \ln x)=\sum_{(i=S)}\left\{x_{h}\right\} /\left\{x_{H}\right\} \\
& =O\left(X^{(1 / 2)+\mathbf{0}}\right)=\left(1-{\eta_{H}}^{2}\right)^{(\mathbf{z} / \mathbf{t})}=1 ;
\end{aligned}
$$

Ensure the smoothness and stability of integer, prime "unitary" and zero (limit) $\{1 / 2\}{ }^{(Z / t)}$ values.

where: $\left\{\mathrm{x}_{\mathrm{h}}\right\}$ is a monomeric prime element, and $\left\{\mathrm{x}_{\mathrm{H}}\right\}$ is a value that is integrated (multiply or continuously) for the listed prime numbers.

\section{(3), Dirichiet function}

The Dirichiet function is the mean value $D(x 0)$ of the calculus equation (polynomial), which is the "characteristic mode function form", which is a mysterious and particularly common research object in number theory. It is required to satisfy the characteristics of Euler product, analytical extension, and Riemann-type functional equations. Including elliptic functions, self-contained functions, and so on. Here, through the processing of polynomial coefficients and combination coefficients, it becomes the characteristic modulus function (average of positive, middle and inverse functions), combined with the periodic elliptic topological function, that is, the logarithmic equation satisfies the relationship of the characteristics of $D(x 0)$ function:

$$
0 \leq \mathbf{D}(x)=\left(1-\eta^{2}\right)^{(Z / t)} \mathbf{D}\left(x_{0}\right) \leq 1
$$

Advantages of choosing a eigenmode function:

(a) The polynomial combination coefficient is known, and the combination of all the sub-items is not forced.

(b) Solving the reciprocal, relative symmetry, and isomorphic uniformity of each sub-item in the element combination. The eigenmode form is invariant with the logarithm of the circle. 


\section{(4), L-function}

:In general, the L-function source consists of two categories: an arithmetic L-function and a self-defending L-function. The two are closely related, according to the PR Langlands conjecture: in general terms, all meaningful $L$ - The functions are all from the self-defending L-function. There are three main parts:

(1) Analytic extension, functional equation: This is easier to obtain for a general self-contained L-function, but the part of the L-function of arithmetic is not easily available.

(2) Distribution of zero points: non-zero region, Riemann's conjecture and generalized Riemann conjecture problem; under the assumption of Riemann's conjecture, the distribution of the imaginary part of the zero point is related to the random matrix and so on.

(3) Values of special points: center value, critical point, value of the whole point, the number of poles, etc. There are also many conjectures, such as BSD conjecture, class number problem, Deligne conjecture, Beilinson conjecture, Goldfeld conjecture and circle pair Number contact. Here is the following

Reciprocity of L-functions under finite regularization conditions: :

$$
\begin{aligned}
& \mathbf{L}(s x)^{(Z / t)}=\sum n^{-s} X(n)=\mathbf{L}_{\mathrm{o}}(s x)^{+(Z / t)} \cdot \mathbf{L}_{e}(s x)^{-(Z / t)} \\
& =\left(C_{( \pm S \pm p}\right)^{+1} X(n)^{+s}\left(C_{( \pm S \pm p}\right)^{-1} X(n)^{-s} \\
& =\left\{X_{\mathrm{o}}(n)\right\}^{+s}\left\{X_{\mathrm{o}}(n)\right\}^{-S} \\
& \mathbf{L}_{\mathbf{0}}(S X)=\left(1-\eta^{2}\right)^{K(Z / t)} \mathbf{L}(S X) ;(K=+1,0,-1)
\end{aligned}
$$

(1) When: $(K=+1)$ the average of the positive function is:

$$
\left\{\mathrm{X}_{0}(\mathrm{n})\right\}^{+S}=\mathrm{L}_{0}(s X)^{(Z / t)}=\left[\sum_{i=( \pm \pm \pm p)}\left(1 / C_{( \pm \leq \pm p}\right)^{+1}\left\{\prod x_{p}^{+1}\right\}\right]^{+1}=\prod\left(1-X(p) p^{+s}\right)^{+1} ;
$$

(2) When: $(K=-1)$ the average of the negative function is:

$$
\begin{aligned}
& \left\{\mathrm{X}_{0}(\mathrm{n})\right\}^{-S}=L_{e}(S X)^{(Z / t)}=\left[\sum_{i=\{ \pm \leq \pm p)}\left(1 / C_{( \pm S \pm p)}\right)^{-1}\left\{\prod x p^{-1}\right\}\right]^{-1}=\prod\left(1-X(p) p^{-s}\right)^{-1} ; \\
& 0 \leq\left(1-\eta^{2}\right)^{K(Z / t)} \leq 1 ;\left(1-\eta^{2}\right)^{K(Z / t)}=\{0,(1 / 2), 1\}^{K(Z / t)} ;
\end{aligned}
$$

Equations (11.6)-(11.9) satisfy the requirements of (all meaningful) L-functions and are closely related to the establishment of the logarithm of the circle.

\subsection{Unification of traditional functions and circular logarithm algorithms}

The algorithm of the function involves the algorithm design and the basic structure of the algorithm, the algorithm design and the programming language. Or the algorithm has developed many tools. The algorithm can be unified by the logarithm of the circle.

\section{(5) Lie groups and Lie algebras}

Li Qun and Lie algebra describe the Newton-Eulerian equation and the Lagrange equation, and obtain the recursive formula of the single dynamic link of the robot dynamics in the joint space and the operating space and the matrix expression of the whole system dynamics equation. In particular, the so-called Lie group method was born under the demand of dynamic problems, which can be discretized in a curved space. That is to say, "the curved space of the logarithm of the circle is adapted to the Lie group method". 
When: a mathematical model of two interacting spin $1 / 2$ particle systems with a variable varying on the $\mathrm{SU}(4)$ of the Lie group. Adapt to the two (rotation and) calculation results of the logarithmic equation. It is contained in a multi-level parallel/serial circular logarithmic equation. Based on the calculus equation with the form of two equilibrium sums of $\{0,2\} K(Z / t)$, the concept of " $i$ complex number" is abolished to calculate the real function, and the arithmetic symbol replaces the logical symbol of the Lie group. The unification of the algorithm is achieved by the relationship between the logarithm of the circle and the Lie group algebra:

$$
\{X, Y\}=\{x y-y x\}^{K(Z \pm \Omega \pm N \pm 2) / t}=\left(1-\eta_{[x y]}\right)^{K(Z \pm \Omega \pm N \pm 2) / t}\left\{X_{0}, Y_{0}\right\}
$$

Where: $\left(1-\eta_{[x y]}\right)^{K(Z \pm \Omega \pm N \pm 2) / t}$ includes the logarithmic equation of the circle of vector, linear, rotation, multilevel, and surface.

\section{(6) Automorphic function}

Self-defense function theory is the application of group theory in function theory in the nineteenth century. Its theory is the product of the intersection of geometry, algebra, complex analysis and differential equation analytic theory. Among them, the self-defense function is a generalization of the concepts of circular function, hyperbolic function and elliptic function, which embodies the unity of mathematics.

In the practice of that era (sail, diaphragm, planetary motion), mathematicians James Bernulli, Bessel, D. Bernulli, Euler, Fourier, Poisson, Lame, Legendre, Weber, Gauss et al. proposed many types of secondorder linear differentials. equation. The most important of these is the hypergeometric equation. It is a second-order linear ordinary differential equation with singularities of 0,1 , and infinity. Euler gave the series solution; Gauss carefully studied the convergence and deeply understood its nature. In 1865 , the problem of studying nth order differential equations was proposed.

Now, for higher-order partial differential equations, the integer solution of an algebraic integer equation is obtained by the logarithm of a circle. It involves the finite transformation group, the combination topology, the eigenmode function form, and the higher-order circular logarithm equation further normalizes the circular logarithmic linear equation that is isomorphic to completeness, and calmly converts the higher-order differential nonlinear problem into the first order. Linear problem, the realization of the unity with the self-defense function.

$$
S(x)=\left(1-\eta^{2}\right)^{K(Z \pm \Omega \pm N \pm p) / t}\{0,2\}^{K(Z \pm \Omega \pm N \pm p) / t}\left\{S\left(x_{0}\right)\right\}
$$

Where:(1-n[xy]2)K(Z $\pm \Omega \pm N \pm p) /$ tincludes the logarithm and topology of the multi-level symmetric and asymmetrical curved surfaces. $\{S(x) 0\}$ represents a eigenmode function (function average).

\section{(7) finite element function}

In 1960, Kraft proposed the finite element method, which approximated the continuum mechanics problem with a finite joint intersection to form a unit body, and defined the function on a unit domain of a simple geometric shape (such as a triangle or an arbitrary quadrilateral in a two-dimensional problem). The slice function is called the particle network method, which uses a limited number of unknowns to approximate an infinitely unknown real system. Become a simple "point and strain" for discrete calculations 
In the logarithmic calculation, it is reflected as "the center point is not moving (called the constant eigenmode function average), the boundary is called the strain), and the boundary is movable" method. In other words. The multi-element second-order partial differential equation can directly pass the circular logarithmic equation formed by the boundary value $\left\{D_{\mathrm{uv}}\right\}$ of the circular logarithmic calculus and the unknown variable $\left\{X_{\mathrm{uv}}\right\}$ and the known coefficients $\{B\},\{C\},\{\ldots\}$. Exact solution.

$$
\Omega^{e}=\sum_{(i=N \pm p)}^{(Z \pm \Omega)} \Omega=\left(1-\eta_{u v}{ }^{2}\right)^{K(Z \pm \Omega \pm N \pm 2) / t}\{0,2\}^{K(Z \pm \Omega \pm N \pm 2) / t}\left\{K^{e} D_{0 u v}\right\} ;
$$

Where: Mechanical parameters of any phase of $K^{e} ;\left\{D_{0 u v}\right\}$ finite element mechanics (matrix) eigenmode function.

\section{(8), functional analysis}

Functional analysis is a mathematical division formed in the 1930s. It is developed from the study of variational problems, integral equations and theoretical physics. They are analytical geometry and mathematical analysis of infinite dimensional vector space, which can be written as high-dimensional calculus equations. Further converted into a circular logarithmic equation and a eigenmode function, the convergence of the function columns in the function space is different by the property power $(K=+1,0,-$ 1) (such as point-by-point convergence, uniform convergence, weak convergence, etc.) ), and a description of the different topologies of the function space.

Functional analysis contains most types of problems in representation theory.

Soft analysis topology group, topological ring and topological vector spaceThe geometry of the Banach space is represented by a series of work by Jean Bourgain.

Non-commutative geometry, including the Alain Connes sub-work, is based on the results of George Mackey's ergodic theory.

For the above functional theory, write the abstract circular logarithm theory and eigenmode under equilibrium conditions

$$
\Omega(x)=\left(1-\eta_{[x y]}{ }^{2}\right)^{K(Z \pm S \pm N \pm p) / t}\{0,2\}^{K(Z \pm S \pm N \pm p) / t}\left\{\Omega\left(x_{0}\right)\right\} ;
$$

where: $\left.\left(1-\eta_{[x y]}\right]^{2}\right)^{K(Z \pm S \pm N \pm p) / t}$ contains any finite circular logarithm of the infinite multi-level functions " symmetry and asymmetry" or "equations and inequalities" With topology. $\left\{\Omega(\mathrm{x})_{0}\right\}$ represents a spatial (event, field) eigenmode function (average of positive, medium, and inverse functions) composed of infinite elements.

\section{(9) String theory - membrane theory}

String theory - membrane theory is a theoretical physics theory. The physical model in theory holds that the most basic unit that constitutes all matter is a small "energy string" or "energy film (field)", which is as large as the interstellar galaxy, as small as electrons, protons, and quarks. This consists of an "energy line" of two-dimensional time and space. The current calculations can't get rid of the interference of specific elements, and there are many mathematical models that bring difficulties in unified computing. The logarithmically extracted eigenmode function is normalized to merge the one-dimensional and twodimensional states, which becomes the infinite expansion of the string theory-membrane theory. Convert 
any arbitrary nonlinear problem into an isomorphic chord-membrane problem, and realize the unity of circular logarithm and string theory-membrane theory

The logarithm of the circle also believes that the wireless multidimensional space is an objective existence, there is no so-called "space shrinkage", which can be normalized in calculation. They are multi-level (nonlinear membranes and linear chords) with isomorphic time calculation consistency. Through the unrelated mathematical topology model of any finite boundary, the various forces mentioned in the string theorymembrane theory are unified with the natural rules.

$$
W(x)=\left(1-\eta_{[x y]}{ }^{2}\right)^{K(Z \pm S \pm N \pm p) / t}\{0,2\}^{K(Z \pm S \pm N \pm p) / t} W\left(x_{0}\right) ;
$$

Where: $\left(1-\eta_{[x y]}\right]^{K(Z \pm S \pm N \pm p) / t}$ contains the circular logarithm and topology of finite and multi-level symmetric and asymmetrical curved surfaces. $\mathrm{W}(\mathrm{x})_{0}$ represents a eigenmode function (function average). $\mathrm{P}=1$ : indicates string theory-grain wave curve; $\mathrm{P}=2$ : indicates film theory-grain wave surface.

\section{Partial Differential Equations-Polynomial-Blockchain and Circular Logarithm Algorithm}

Partial differential polynomial equations, polynomials, and blockchains can all be solved by a circular logarithmic algorithm, including the mathematical basis of quantum computing. Specific steps:

(1) Apply the discrete type to determine the eigenmode function form of the infinite rational point and the known boundary condition or the corresponding program principle of the traditional computer.

(2) Introduce any finitely rational circular logarithmic topological range, which becomes an additional circular logarithmic entanglement type program, and obtain the values of each regularization coefficient of each calculus polynomial.

(3) Solve the construction principles of manual calculation or quantum computer program and architecture, chip simplification.

\subsection{Logarithmic Equation and Circular Logarithm Algorithm}

(A), high order partial differential dynamic equation

Each scientific field is an expression that is bounded by scientific elements according to experimental results, and is written as a circular logarithmic calculus polynomial equation. The circular logarithm algorithm here is that the logarithmic equation is closely related to the eigenmode function form as a whole. 10.14738/aivp.81.7558

Calculation method. Proceed as follows:

(1), the number of unknown variables $\{X\}$ elements and the composition rules (password notification), you can know the relationship between the equation coefficients and the item order, and list the equations.

(2), known conditions:

$$
\left\{D_{0}\right\}^{(Z / t)}=\left\{B / C_{K(Z \pm S \pm N \pm 1)}\right\}^{(Z / t)} ; \ldots ;\left\{D_{0}\right\}^{(Z / t)}=\left\{P / C_{K(Z \pm S \pm N \pm p)}\right\}^{(Z / t)} ;
$$




$$
\begin{aligned}
& \{X \pm D\}^{(Z / t)}=A x^{K(Z \pm S \pm N-0) / t}+B x^{K(Z \pm S \pm N-1) / t}+C x^{K(Z \pm S \pm N-2) / t}+\ldots \\
+ & P x^{K(Z \pm S \pm N-p) / t}+Q x^{K(Z \pm S \pm N-q) / t}+S D^{K(Z \pm S \pm N+S) / t} \\
= & \left(1-\eta^{2}\right)^{(Z / t)}\{0,2\}^{(Z / t)}\left\{D_{0}\right\}^{(Z / t)}
\end{aligned}
$$

The high-order partial differential dynamic equation solves many uncertain factors and is computationally heavy. Through the logarithm algorithm with the circle

Solve the problem of arbitrary algebraic integers by normalization and equivalent replacement principle, for architecture simplification and chip development The basics of mathematics.

(B), blockchain and circular logarithm algorithm

The essence of the blockchain is the parallel/serial dynamic equation.

(a) Parallel equation: form a positive function eigenmode

$$
\{H\}=\left\{H_{A}+H_{B}+\ldots+H_{P}+\ldots\right\}
$$

(b) , serial equation: composing complex function eigenmode

$$
\{H\}=\left\{H_{A} \cdot H_{B} \cdot \ldots \cdot H_{P}+\ldots\right\}
$$

Written as: $\{H\}^{K(Z \pm H)}=\sum_{(i=H)}^{K(Z)}\left\{H_{A}{ }^{k}+H_{B}{ }^{k}+\ldots+H_{P}{ }^{k+} \ldots\right\}^{K(Z \pm H \pm N \pm p)}$;

$$
\begin{aligned}
& \left\{X_{H} \pm D_{H}\right\}^{K(Z \pm H) / t}=A x^{K(Z \pm A \pm N \pm p) / t}+B x^{(Z \pm B \pm N \pm p) / t}+\ldots+P x^{(Z \pm P \pm N \pm p) / t}+\ldots \\
= & \left(1-\eta_{A}^{2}\right)^{K(Z \pm A) / t}\{0,2\}^{K(Z \pm A) / t}\left\{H_{0 A}\right\}^{K(Z \pm A) / t} \\
+ & \left(1-\eta_{B}^{2}\right)^{K(Z \pm B) / t}\{0,2\}^{K(Z \pm B) / t}\left\{H_{0 B}\right\}^{K(Z \pm B) / t}+\ldots \\
+ & \left(1-\eta_{P}^{2}\right)^{K(Z \pm P) / t}\{0,2\}^{K(Z \pm P) / t}\left\{H_{0 P}\right\}^{K(Z \pm P) / t}+\ldots \\
= & \left(1-\eta_{(S \pm H)}\right)^{K(Z \pm H) t}\{0,2\}^{K(Z \pm H) / t}\left\{D_{0}\right\}^{K(Z \pm H) / t} ;
\end{aligned}
$$

The three zero-point equilibrium states of $\{0,2\}^{\mathrm{K}(\mathrm{Z} \pm \mathrm{H})}$ in the formula (12.1) (12.2):

(1), zerobalance: odd balance $\{0\}^{\mathrm{K}(\mathrm{Z}+\mathrm{H})}$. Indicates the singular balance of spin, mutation, zero, vortex, vortex, and galaxies.

$$
\{X-D\}^{(Z / t)}=\left(1-\eta^{2}\right)^{(Z / t)}\{0\}^{(Z / t)}\left\{D_{0}\right\}^{(Z / t)} ;
$$

(2), large balance: even balance $\{2\}^{\mathrm{K}(\mathrm{Z} \pm \mathrm{H})}$. Represents the even balance of the male spin, radiation, qubits, and galaxies.

$$
\{X+D\}^{(Z / t)}=\left(1-\eta^{2}\right)^{(Z / t)}\{2\}^{(Z / t)}\left\{D_{0}\right\}^{(Z / t)} ;
$$

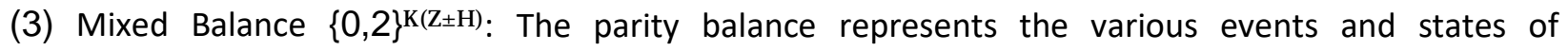
parallel/serial.

$$
\{X+D\}^{(Z / t)}=\left(1-\eta^{2}\right)^{(Z / t)}\{2\}^{(Z / t)}\left\{D_{0}\right\}^{(Z / t)}
$$


Regardless of whether the combination is parallel (continuously added) or serial (multiplied), it can be converted into a eigenmode function (positive, intermediate, inverse averaging function) with unlimited capacity privacy, anonymity (digitization), and its corresponding arbitrary The circular logarithmic topology [0 or 1] has "decentralization, openness, independence, security" (digitization). The statistical analysis of the unrelated mathematical model expressed as a logarithm between [0 to 1$]$.

In particular, "large zero balance" cancels the "imaginary number $\mathrm{i}$ ", making the imaginary and complex functions in traditional mathematics become complete real functions. Conveniently handle infinite dimensional calculus equations and solutions.

\section{2 solving elements:}

According to the zero-point equilibrium equation of the calculus polynomial equation, the order value $(N=1) B$ (the second coefficient) is generally known to obtain $\left\{D_{0}\right\}=B / C_{K(S \pm N \pm 1)}$; coefficient,

For example: $(N=2)$ get $\{D 0\}=C / C_{K(S \pm N \pm 2)},(N=p)$ get $\left\{D_{0}\right\}=C / C_{K(S \pm N \pm p)}, \ldots$, also adapt to any height The solution of order multivariate $\mathrm{N}$-order partial calculus equations and ordinary differential equations.

Solving has the following steps:

(a), according to: element composition rules (password notification / privacy),

$$
\begin{aligned}
& \left\{X^{-1}\right\}^{K(Z \pm S \pm N) / t}=\left\{^{K(S \pm N)} \sqrt{ } D\right\}^{K(Z \pm S \pm N) / t} ; \\
& \left\{X_{0}\right\}^{K(Z \pm S \pm N) / t}=\left\{D_{0}\right\}^{K(Z \pm S \pm N) / t} \\
& \left(1-\eta_{H}{ }^{2}\right)=\sum_{(i=p)}^{K(Z \pm N)}\left(1-\eta_{h}{ }^{2}\right)=1 ;
\end{aligned}
$$

(b) make: $\left(1-\eta^{2}\right)^{K(Z / t)}=\left[\left\{X^{-1}\right\} /\left\{X_{0}^{+1}\right\}\right]^{K(Z / t)}=\left\{{ }^{K(S \pm N)} \sqrt{ } D / D_{0}\right\}^{K(Z / t)}=1$;

(c) get: $\quad\left\{X^{-1}\right\}=\left(1-\eta^{2}\right)^{K(Z / t)}\left(1-\eta_{H}^{2}\right)^{K(Z / t)}\left\{D_{0}\right\}^{K(Z / t)}$;

(d) Calculate individual elements: $\left[\left(\eta_{h i}{ }^{2}\right) \operatorname{or}\left(\eta_{h i}\right)\right]=\left[\left(\eta_{H}{ }^{2}\right) \operatorname{or}\left(\eta_{H}\right)\right]\left\{D_{0}{ }^{2}, D_{0}\right\}$;

\section{3 the logarithm of the circle and the coordinate system and the jump}

(1) The arbitrary function $\{X\}^{(Z / t)}$ is expressed by the logarithm of the circle $\left(1-\eta^{2}\right)^{(Z / t)}$, and the natural rule of change is unchanged. Any starting point between the ' 0 to 1 ' of the start point and the end point can be used as The origin of the coordinates is independent of the coordinate selection.

Three-dimensional coordinates: Taking the center point of any balance as the coordinate origin, the threedimensional rectangular coordinates $(\mathbf{i}, \mathbf{j}, \mathbf{k})$ are introduced, and the spheres (outside) can be mapped to the coordinate axes. Such as each sub-item in the respective three-dimensional space coordinates Cartesian coordinates, Hilbert space ... and so on.

$$
\left(1-\eta^{2}\right)^{K(Z / t)}=\left(1-\eta_{[x]}{ }^{2}\right)^{K(Z / t)} i+\left(1-\eta_{[y]}{ }^{2}\right)^{K(Z / t)} j+\left(1-\eta_{[z]}{ }^{2}\right)^{K(Z / t)} k
$$

(2) Three-dimensional spherical coordinates: Taking the center point of any balance as the coordinate origin, three-dimensional right-angle spherical coordinates are introduced ( $[x y]$ corresponds to the normal axis k; [yz] corresponds to the normal axis $\mathbf{i}$; [zx] corresponds to the normal axis $\mathbf{j}$ ), its sphere (outside) can be mapped to the coordinate axis, such as Einstein's electric equation - Maxwell's 
electromagnetic equation, spin equation and so on. Each sub-item has its own three-dimensional spherical coordinates $(\mathbf{i}, \mathbf{j}, \mathbf{k})$ :

$$
\left(1-\eta_{u v}{ }^{2}\right)^{(Z / t)}=\left(1-\eta_{[Z Y]}{ }^{2}\right)^{(Z / t)} \mathbf{i}+\left(1-\eta_{[x]}{ }^{2}\right)^{(Z / t)} \mathbf{j}+\left(1-\eta_{[x]]}{ }^{2}\right)^{(Z / t)} \mathbf{k}
$$

(3) Function (high-order partial calculus dynamic equation)

$$
\left(1-\eta^{2}\right)^{K( \pm \Delta N \pm \Delta p)}=\left(1-\eta^{2}\right)^{K(Z \pm S \pm \Delta N \pm \Delta \mathbf{p})} /\left(1-\eta^{2}\right)^{K(Z \pm S \pm N \pm \mathbf{p})} ;
$$

(4) Lifting order hierarchy and energy orbital transition of function (high-order partial calculus equation)

$$
\boldsymbol{\Sigma}_{(i=( \pm N \pm p))}^{K(Z \pm S \pm N \pm p)}\left(1-\eta^{2}\right)^{K( \pm \Delta N \pm \boldsymbol{\Delta} p)}=\{2\}^{K( \pm \boldsymbol{\Delta} N \pm \boldsymbol{\Delta} p)} ;
$$

Where: $\Delta \mathrm{N}=0,1,2,3, \ldots ; \Delta \mathrm{p}=0,1,2,3, \ldots$ natural number

\section{4 algorithm unified}

For functions consisting of number theory, algebra, geometry, group theory, etc. (high-order partial calculus dynamics equation) $\mathrm{W}=\{\mathrm{X}\}(\mathrm{Z} \pm \mathrm{S} \pm \mathrm{N}) / \mathrm{t}$ can be an infinite program element composed of infinite elements (integer, fraction, The primed and irrational eigenmode functions, through close association with the logarithmic topology of any finite circle, become an unrelated mathematical model, solved between [0 and 1$]$, and obtain an exact solution of any one element:

$$
\begin{gathered}
W=\left(1-\eta^{2}\right)^{(Z / t)} W_{0} \\
0 \leq\left(1-\eta^{2}\right)^{(Z / t)}=\left(1-\eta_{T}{ }^{2}\right)^{(Z / t)}=\left(1-\eta_{H}{ }^{2}\right)^{(Z / t)}=\left(1-\eta_{K}{ }^{2}\right)^{(Z / t)} \leq 1 ;
\end{gathered}
$$

"W, $\left(1-\eta^{2}\right),(Z / t), W_{0}$ " consists of four sets of high-order partial calculus dynamic equations with selfconsistent, concise, elegant and practical characteristics.

where: $\mathrm{W}, \mathrm{W} 0$ and $\{X\}^{(Z / t)}$ or $\left\{D_{0}\right\}^{(Z / t)}$ have events, event mean, $\left(1-\eta^{2}\right)^{(Z / t)}$ circular logarithmic dynamics equation, ( Or relativistic structure, supersymmetric element matrix, mathematical relativity), combined with time factor, becomes a high-order calculus dynamic equation, and integrates entanglement calculation and discrete calculation. Get reliable, scientific integer solutions.

\section{The unity of circular logarithm and contemporary physics formula}

The relationship between the logarithm of the circle and some contemporary physical formulas reflects the unified rules of nature. Many physical formulas are the circular logarithmic partial calculus dynamic equations written by the mathematical summaries of physics experiments. Different physical domain formulas are unified by circular logarithms and eigenmode functions. Explain the high-order conditions: original function (zero calculus order), velocity (first order), acceleration (second order), super acceleration (super-order, hypergeometry, high-order algebra), wave-grain, mass-space- Energy-time, etc., there is no essential difference except for the invariant eigenmode function form (including the interaction range and mechanical parameters of each corresponding domain) 


\subsection{Explain the unity of Einstein's gravitational relativity and Newton's gravitational formula.}

The mass-energy (wave-spoke, frequency) mathematical model of gravitational star interaction has Newton's gravitation formula and Einstein's gravitational relativity: classical gravity satisfies spatial linear distance; relativistic gravity satisfies spatial curvature. It is a multi-strand parallel/serial second-order partial differential dynamic equation. Power function $Z=K(Z \pm \Omega \pm N \pm p) / t ;(p)$ combination condition( $N \geq 3$ ) (super-order), ( $\mathrm{N}=-2)$ (second order), $(\mathrm{N}=-1)$ (first order), $(\mathrm{N}=0)$ (original The combination of functions) is uniformly converted into a circular logarithmic equation

$$
\begin{aligned}
& \left\{\left(M_{g} R\right)\right\}^{K(Z \pm \Omega \pm 2 \pm p) / t}=\left\{M_{g} R^{2}\right\}^{K(Z \pm \Omega \pm 1 \pm p) / t} \\
& =\left(1-\eta_{u v}{ }^{2}\right)^{K(Z \pm \Omega-2 \pm p) / t}+\left(1-\eta_{u v}{ }^{2}\right)^{K(Z \pm \Omega-1 \pm p) / t}+\left(1-\eta_{u v}{ }^{2}\right)^{K(Z \pm \Omega-0 \pm p) / t} \\
& =\left(1-\eta_{u v}{ }^{2}\right)^{K(Z \pm \Omega-2 \pm p) / t}\{0,1\}^{K(Z \pm \Omega-2 \pm p) / t}\left\{M_{g} R\right\}^{K(Z \pm \Omega-2 \pm p) / t} \text {; } \\
& \left(1-\eta^{2}\right)^{K(Z / t)}=\left(1-\eta_{[x]}^{2}\right)^{K(Z / t)} i+\left(1-\eta_{[y]}^{2}\right)^{K(Z / t)}, j+\left(1-\eta_{[z]}{ }^{2}\right)^{K(Z / t)} k \text {; } \\
& \left(1-\eta_{u v}{ }^{2}\right)^{K(\mathbf{Z} / \mathbf{t})}=\left(1-\eta_{[z y]}{ }^{2}\right)^{(\mathbf{Z} / \mathbf{t})} \mathbf{i}+\left(1-\eta_{[z x]}{ }^{2}\right)^{(\mathbf{Z} / \mathbf{t})} \mathbf{j}+\left(1-\eta_{[x y]}{ }^{2}{ }^{(\mathbf{Z} / \mathbf{t})} \mathbf{k}\right. \text {; }
\end{aligned}
$$

Among them: gravitational mass $\mathrm{Mg}$ contains gravitational parameters; $\left(1-\eta_{\mathrm{uv}}{ }^{2}\right)^{\mathrm{K}(\mathrm{Z} \pm \mathrm{S}-2 \pm \mathrm{p}) / \mathrm{t} T h e}$ multialtitude circular logarithmic equation is a five-dimensional dynamic equation composed of two threedimensional motions. $K=(+1,0,-1)$ respectively denotes centripetal force and centrifugal force, positive gravitational force and antigravity, and action space; $\{0\}^{\mathrm{K}(\mathrm{Z} \pm \Omega+N \pm 2) / \mathrm{t}}$ balanced spin motion; $\{1\}^{\mathrm{K}(\mathrm{Z} \pm \Omega+N \pm 2) / \mathrm{t}}$ balanced commutation motion; $\{2\}^{\mathrm{K}(\mathrm{Z} \pm \Omega+\mathrm{N} \pm 2) / \mathrm{t}}$ balanced “coil + male” motion or vortex gravitational wave radiation .

\subsection{Explain the unity of gravitational relativity and Maxwell's electromagnetic force formula.}

In the discovery of gravitational waves in 2015, gravitation and electromagnetic force are both proofs of wave function and wave-particle duality, which makes the gravitational mass and electromagnetic charge have a unified description, and a unified circular logarithmic equation can be established.

(1) Einstein's Gravitational Equation and Circular Logarithmic Equation There are: Einstein's gravitational relativity electric equation [6] conversion circle logarithm

$$
\begin{aligned}
& \left\{M R_{g[X]}{ }^{2}\right\}^{(\mathrm{Z} / \mathrm{t})}=\left\{r_{N}{ }^{2}\right\}^{(\mathrm{Z} / \mathrm{t})}-\left\{r_{M}{ }^{2}\right\}^{(\mathrm{Z} / \mathrm{t})}=\left(1-\eta_{[Z Y]}{ }^{2}\right)^{(\mathrm{Z} / \mathrm{t})} \mathbf{i}\left\{M_{g} R_{0 x}{ }^{2}\right\}^{(\mathrm{Z} / \mathrm{t})} \\
& \left\{M R_{g[Y]}{ }^{2}\right\}^{(Z / t)}=\left\{r_{L}{ }^{2}{ }^{(Z / t)}-\left\{r_{N}{ }^{2}\right\}^{(Z / t)}=\left(1-\eta_{[X Z]}{ }^{2}\right)^{(Z / t)} \mathbf{j}\left\{M_{g} R_{0 y}{ }^{2}\right\}^{(Z / t)}\right. \\
& \left\{M R_{g[Z]}{ }^{2}\right\}^{(\mathrm{Z} / \mathrm{t})}=\left\{r_{M}{ }^{2}\right\}^{(\mathrm{Z} / \mathrm{t})}-\left\{r_{L}{ }^{2}\right\}^{(\mathrm{Z} / \mathrm{t})}=\left(1-\eta_{[\mathrm{YX}]}{ }^{2}\right)^{(\mathrm{Z} / \mathrm{t})} \mathbf{k}\left\{M_{g} R_{0 z}{ }^{2}\right\}^{(\mathrm{Z} / \mathrm{t})} \text {; } \\
& \left(1-\eta_{[Z Y]}{ }^{2}\right)^{(\mathbf{z} / t)} \mathbf{i}=\left[\left(1-\eta_{N}{ }^{2}\right)^{(\mathbf{z} / t)}-\left(1-\eta_{M}{ }^{2}\right)^{(\mathrm{z} / \mathrm{t})}\right]=\left(1-\eta_{[X]}{ }^{2}\right)^{(\mathrm{z} / \mathrm{t})} \\
& \left(1-\eta_{[X Z]}{ }^{2}\right)^{(\mathbf{Z} / t)} \mathbf{j}=\left[\left(1-\eta_{L}{ }^{2}\right)^{(\mathbf{Z} / t)}-\left(1-\eta_{N}{ }^{2}\right)^{(\mathrm{Z} / t)}\right]=\left(1-\eta\left[_{Y]}{ }^{2}\right)^{(\mathrm{Z} / \mathrm{t})}\right. \\
& \left(1-\eta_{[Y X]}{ }^{2}\right)^{(\mathrm{Z} / t)} \mathbf{k}=\left[\left(1-\eta_{M}{ }^{2}\right)^{(\mathrm{z} / \mathrm{t})}-\left(1-\eta_{L}{ }^{2}\right)^{(\mathrm{z} / \mathrm{t})}\right]=\left(1-\eta\left[_{\mathrm{Z}]}{ }^{2}\right)^{(\mathrm{z} / \mathrm{t})}\right. \text {; }
\end{aligned}
$$

(2) Maxwell's electromagnetic field equation and circular logarithmic equation

$$
\begin{aligned}
& \left\{Q R_{e X}{ }^{2}\right\}^{K(Z / t)}=\left(1-\eta_{X}{ }^{2}\right)^{Z} \mathbf{i}\left\{R_{e X}{ }^{2}\right\}^{K(Z / t)}=\left(1-\eta_{[X Z]}{ }^{2}\right)^{Z} \mathbf{i}\left\{Q R_{e X}{ }^{2}\right\}^{K(Z / t)} \\
& \left\{Q R_{e X}{ }^{2}\right\}^{K(Z / t)}=\left(1-\eta_{Y}{ }^{2}\right)^{Z} \mathbf{j}\left\{R_{e X}{ }^{2}\right\}^{K(Z / t)}=\left(1-\eta_{[Z X]}{ }^{2}\right)^{Z} \mathbf{j}\left\{Q R_{e X}{ }^{2}\right\}^{K(Z / t)} \\
& \left\{Q R_{e X}{ }^{2}\right\}^{K(Z / t)}=\left(1-\eta_{Z}{ }^{2}\right)^{Z} \mathbf{k}\left\{R_{e X}{ }^{2}\right\}^{K(Z / t)}=\left(1-\eta_{[X Y]}{ }^{2}\right)^{Z} \mathbf{k}\left\{Q R_{e X}{ }^{2}\right\}^{K(Z / t)} ;
\end{aligned}
$$




\subsection{Explain the unity of Einstein's energy equation}

Einstein combines mass-frequency-space-time to complete the energy equation ${ }^{[6]}$, but the massfrequency has uniformity and non-uniformity. Then the energy equation: when the spatial field is infinite or infinitesimal or isotropic particles, the symmetric topological space is invariant to the localized norm, called the localized Abelian norm field;

$$
E=h v=E=M C^{2} ;\left(1-\eta^{2}\right)^{(\mathbf{Z} / \mathbf{t})}=\{0 \text { or } 1\} ;
$$

When: the topological space of arbitrary symmetry and asymmetry, continuous and discontinuous, random and regular, etc. is variable for non-localized norms, called non-Abelian normative field;

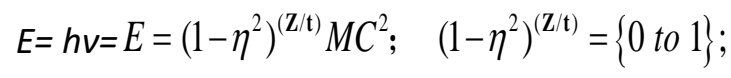

\subsection{Explain $\{\mathrm{MR}\}(\mathrm{z} / \mathrm{t})$ energy symmetry and asymmetry}

The existence condition of asymmetry energy: under the vacuum, the two energy particles $\left(1-\eta^{2}\right)^{\mathrm{K}(\mathrm{z} / \mathrm{t})}$ collide, and the excitation produces a non-localized norm variable asymmetry super energy $\left(1-\eta^{2}\right)^{-(z / t)}$ and $\left(1-\eta^{2}\right)^{+(z / t)}$. Similarly, the asymmetric super-energy can be inversely aggregated to $\left(1-\eta^{2}\right)^{0(z / t)}$ under vacuum conditions $\left(1-\eta^{2}\right)^{-(z / t)}$ and $\left(1-\eta^{2}\right)^{+(z / t)}$. The overall satisfaction of energy conservation.

(1), $\{M R\}^{(z / t)}$ local energy conservation

$$
\left(1-\eta^{2}\right)^{0(Z / t)}=\left(1-\eta^{2}\right)^{+(Z / t)}+\left(1-\eta^{2}\right)^{0(Z / t)}+\left(1-\eta^{2}\right)^{+(Z / t)}=\{2\}^{0(Z / t)} ;
$$

(2), $\{\mathrm{MR}\}^{(z / t)}$ local energy is not conserved

$$
\begin{aligned}
& \left(1-\eta^{2}\right)^{0(\mathbf{Z} / \mathbf{t})}=\left(1-\eta^{2}\right)^{+(\mathbf{Z} / \mathbf{t})}+\left(1-\eta^{2}\right)^{-(\mathbf{Z} / \mathbf{t})}=1 ; \\
& \left(1-\eta^{2}\right)^{-(\mathbf{Z} / \mathbf{t})} \geq\left(1-\eta^{2}\right)^{0(\mathbf{Z} / \mathbf{t})} \geq\left(1-\eta^{2}\right)^{+(\mathbf{Z} / \mathbf{t})} ;
\end{aligned}
$$

\subsection{Explain what Einstein calls "ghost particles".}

There is no essential difference between the speed (linear), acceleration (second-order linear), and superacceleration (superlinear) produced by "mass-space" under high-order calculus.

(1), $\{M R\}$ and the equilibrium $\left\{M_{0} R_{0}\right\}$ have reciprocal symmetry and asymmetry, and the asymmetric field balance center point converts the movement self-consistent to relative symmetry. Explain that the field space has uniform symmetry only when it is infinite (small). Therefore, the two particles in the entangled state have the same induction phenomenon, called the covariation principle.

$$
\begin{gathered}
|\eta|^{+(Z / t)}=|\eta|^{-(Z / t)} ; \\
|\eta|^{+(Z / t)}+|\eta|^{-(Z / t)}=\{1\}^{-(Z / t)} ; \\
|\eta|^{+(Z / t)}+|\eta|^{0(Z / t)}+\left|\eta^{-(Z / t)}\right|=\{0,1 / 2,1,2\}^{(Z / t)} ;
\end{gathered}
$$

(2) Invariance of the equilibrium of energy eigenmode $\{M R\}^{K(z / t)}$ and $\left\{M_{0} R_{0}\right\}^{K(z / t)}$ under entanglement

$$
E=\left|\left(1-\eta^{2}\right)^{(\mathbf{Z} / \mathbf{t})}\right|=\left\{M_{0} R_{0}^{2}\right\}^{K(Z \pm S \pm N \pm p) / t} ;
$$

Mass and space are in their corresponding field spaces, the particle $\{\mathrm{M}\}^{\mathrm{K}(\mathrm{z} / \mathrm{t})}$ is infinitesimally small, and the induced space $\left\{R^{2}\right\}^{K(z / t)}$ is infinite. The opposite is also true. There is no inevitable direct connection 
with the speed of light, or a natural phenomenon of infinitesimal "wave-particle" transmission and inductive energy in an infinite universe.

$$
\left(1-\eta^{2}\right)^{+(Z / t)} \rightarrow 0,\left(1-\eta^{2}\right)^{-(Z / t)} \rightarrow \infty
$$

\subsection{Explaining Mayorana Fermi}

The 2019 "SCINCE" report: The experimental focus of the team, such as Alex Matos-Abiagued, is placed on the Mayorana Fermi, a substance with positive and negative particles, of the same mass and opposite physical charge. The transition of the quantum state from the traditional state to the new topological state is analyzed collectively, and the energy barrier between these states is measured. These transition characteristics are also measured directly, eventually forming a "quantum entangled state of two opposite states".

$$
W(\cdot)=\left\{\left(1-\eta^{2}\right)^{+K(Z \pm S \pm N \pm p) / t}+\left(1-\eta^{2}\right)^{0 K(Z \pm S \pm N \pm p) / t}+\left(1-\eta^{2}\right)^{-K(Z \pm S \pm N \pm p) / t}\right\} W_{0}(\cdot) ;
$$

Equation (14.15) has a positive-middle inverse eigenmode function and a circular logarithmic topological state transition phenomenon.

\subsection{Interpret "0 or 1" and "0 to 1" of infinite "1 qubit" state}

According to the 2019 "NATURE" report: Google announced that it successfully created 54 qubit computers, which fulfilled the task that traditional computers could not. In the world's largest computer that required 10,000 years of computing, the quantum computer took only 200 seconds to gain world wonder.

From the point of view of the circular logarithm algorithm: for quantum computing is binary, the "1 qubit" state is "0 or 1", which belongs to the discrete state calculation and depends on the infinitely superposed linear equation. If the "1 qubit" (different size) state is " 0 to 1 ", the experimental results of the new topological superconducting phase are different for the experimental sequence parameters. Will this quantum computer effect be compromised? The key is the mathematical foundation of the "unique superposition state" ? Why is "the quantum bit computer temperature is kept at an absolute zero or so in a normal temperature environment? Why is it that the mathematical foundation and application have some limitations?"

Using the logarithm algorithm: $\{54\}$ qubits for public quantum computers, even to the "1 qubit" state of hundreds of millions of different sizes, you can use the "student computer" to calculate in parallel in the classroom (or create novel Small-quantity quantum computer) can also be completed in a short time at normal temperature to achieve a magical effect.

Start with the "1 qubit" experimental quantum topology of $(S \geqslant 54)$. According to the known composition conditions and the parallel/serial theorem, the corresponding series of characteristic mode functions and circular logarithmic equations such as " 1 element 54 times, or parallel 16 yuan 3 times, or 9 yuan 6 times" are established. Converting the infinite nonlinear qubit combination topology to the mutual transformation of any finite linear "1 qubit" combination topology (called linear harmonic oscillator and its topological model) by each circular logarithmic equation, with magicality The quick calculation is completed in a short time. 
Known conditions: 54 elements (qubits) and the target's "speed refers to the order of magnitude" to form a rule, balanced boundary conditions $D$. $Z=K(S \pm N \pm 54) / t=\{54\}$ written as $W(\cdot)=\{2\}^{K(Z \pm 54 \pm N \pm p) / t}$, the qubit is

"1 yuan of motion calculation and storage unit" 54 times" partial differential circular logarithmic dynamic equation $\mathrm{W}(\cdot)$. Or write other parallel equations of "speed refers to the order of magnitude".

$$
\begin{aligned}
& W(\cdot)=\{X \pm D\}^{K(Z \pm 54 \pm p) / t} \\
& =A X^{K(Z \pm 54 \pm N \pm 0) / t}+B X^{K(Z \pm 54 \pm N \pm 1) / t}+\ldots+P X^{K(Z \pm 54 \pm N \pm p) / t}+\ldots \pm D \\
& =\left(1-\eta_{54}{ }^{2}\right)^{K(Z \pm 54 \pm N \pm p) / t}\{0,2\}^{K(Z \pm 54 \pm N \pm p) / t}\left\{D_{0}\right\}^{K(Z \pm 54 \pm N \pm p) / t} ; \\
& \left(1-\eta_{54}{ }^{2}\right)^{K(Z \pm 54 \pm N \pm p) / t}=\{X\} / D=\left\{^{K( \pm S \pm 54)} \sqrt{ } \mathbf{D} /\left\{D_{0}\right\}^{K(Z \pm S \pm 54 \pm p) / t} ;\right.
\end{aligned}
$$

(1) Discrete calculation of machine learning corresponding to infinite element eigenmode function form

$$
\left(1-\eta_{54}{ }^{2}\right)^{K(Z \pm 54 \pm N \pm p) / t}=\{0 \text { or } 1\} ;
$$

(2) Entanglement calculation of quantum computers corresponding to arbitrary finite topology of multiple qubits

$$
\left(1-\eta_{54}{ }^{2}\right)^{K(Z \pm 54 \pm N \pm p) / t}=\{0 \text { to } 1\}
$$

(3) Entanglement calculation of quantum computers corresponding to arbitrary finite topology of multiple qubits

$$
\left(X_{p}\right)=\sum_{(i=p)}^{(Z \pm 54) / t}\left(\eta_{54}\right) \cdot\left\{D_{0}\right\}^{( \pm 54 \pm p) / t} ;
$$

Equations (14.16)-(14.20) also adapt to different "qubits" (numerical, algebraic, spatial, group, event) of infinitely arbitrary higher order, invariant conditions in the "quantum bit" eigenmode (total mean) Next, according to the principle of entanglement covariation: two or more particles (mass, distance, energy, velocity, acceleration, super acceleration, etc.) change, not only isomorphism affects adjacent particle changes, but also isomorphism affects overall particle changes, It can be normalized to a linear arithmetic calculation (superimposed state).

\section{Three-body problem}

The three-body problem is the basic mechanical model in celestial mechanics. It refers to the problem that the three masses, the initial position and the initial velocity are arbitrary objects that can be regarded as mass points, and the motion law under the gravitational force between each other. At present, the three-body problem cannot be solved accurately, that is, the mathematical situation of all three-body problems cannot be predicted. Only a few special cases have been studied.

Such as: $\mathrm{m}_{\mathrm{gA}}$ (the moon), $\mathrm{m}_{\mathrm{gB}}$ (the earth), $\mathrm{m}_{\mathrm{gc}}$ (the sun) surround each other (revolution three-dimensional + spin two-dimensional $=$ five-dimensional vortex) movement, there is no repeat(0(3),1-1,2-2, three combinations of $\left(\{2\}^{3}-1=7\right) 7$ motion states, and then surround the fourth (star, or combined multi-body common centroid) $\mathrm{MgD}_{\mathrm{gD}}$ motion.

The energy state $\left\{\mathrm{MgR}_{\mathrm{gR}}\right\}^{(\mathrm{z} / \mathrm{t})}$ is written as a 15-dimensional one-dimensional cubic second-order circular logarithmic partial differential equation. Power function

$$
\left.S=3 \times 5=15 ; \quad N=0,-1,-2 ; p=0,1,2,3 ; \quad K=+1,0,-1_{0}(Z / t)=K(S \pm N \pm p) / t=K(15 \pm 2 \pm 3) / t\right),
$$


their eigenmode function $\mathbf{D}=\left\{M_{g o} R_{O}\right\}^{(z / t)}$ is the equilibrium centroid of the common centroid or field space of various combinations of planets

The calculation step:

(1) determining various invariant eigenmode functions of the combined motions and combinations;

(2) Perform round logarithms and calculations of orbits, speeds, and forces of various planetary combinations;

(3) Calculate the relevant values of each planetary motion according to the logarithmic composition of the unit circle.

(4) Characteristics: The analytic calculation of the [0 to 1$]$ interval without specific quality content is performed under the condition that the eigenmode is unchanged.

$$
\begin{aligned}
& W(\cdot)=\{X \pm D\}^{K(Z \pm 54 \pm p) / t} \\
& =A X^{K(Z \pm 54 \pm N \pm 0) / t}+B X^{K(Z \pm 54 \pm N \pm 1) / t}+\ldots+P X^{K(Z \pm 54 \pm N \pm p) / t}+\ldots \pm D \\
& =\left(1-\eta_{54}{ }^{2}\right)^{K(Z \pm 54 \pm N \pm p) / t}\{0,2\}^{K(Z \pm 54 \pm N \pm p) / t}\left\{D_{0}\right\}^{K(Z \pm 54 \pm N \pm p) / t} \\
& \left(1-\eta_{54}{ }^{2}\right)^{K(Z \pm 54 \pm N \pm p) / t}=\{X\} / D=\left\{{ }^{K( \pm S \pm 54)} \sqrt{ } \mathbf{D} /\left\{D_{0}\right\}^{K(Z \pm S \pm 54 \pm p) / t}\right.
\end{aligned}
$$

Where: the order of the circular logarithmic partial differential equation: $(\mathrm{N}=0,3)$ original function, zero order; $(N=-1)$ first order, velocity; ( $N=-2)$ second order, acceleration, force. In $K=(+1, \pm 0,-1)$ : centripetal force $(k=+1)$, centrifugal force $(k=-1)$, neutral field space $(k \pm 0)$. Einstein's general theory of relativity expresses the positive gravitational force of the surface $(k=+1)$, the antigravity $(k=-1)$, and the neutral field space $(k= \pm 0)$. The calculation of the two is unified.

\section{1 the movement of a single planet}

The movement of a single planet is the basis of the multi-star series, which needs to be solved first, as evidenced below.

Known conditions: single planet mass $\left(\mathrm{m}_{\mathrm{g}}\right.$ ) (including gravitational parameter $\mathrm{g}$ ); around the centroid point position $\mathrm{O}_{\circ}$ and the characteristic mode $\mathbf{D}$ for balanced elliptical motion. The elliptical orbital length axis $R_{(L+M)}$, the eccentricity e, and the initial position $\mathrm{O}_{1}$ (including the azimuth angle $\theta_{1}$ ).

Solve: (1), single planet gravity;

(2) The speed of a single planet;

(3) The elliptical orbit of a single planet (gravitational field).

In particular, the movement of the planet in orbit. The area (energy) swept by the unit time is constant, and the tangential line speed and force on the track change. Due to the entanglement, the male (precession) and the spin are synchronously changed

Let: A single-planetary motion in the infinite galaxies of gravitation, the spin-or-revolution around the centroid for elliptical orbital motion, the average circle radius $R_{0}=(1 / 2)\left(L_{a}+L_{b}\right)$. The energy field $\left\{M_{g} R\right\}^{K(Z \pm S \pm N)}$; wherein: the spin+revolution constitutes two three-dimensional, wherein the revolution and the direction of the plane of the spin plane coincide with a five-dimensional $(S=5)$ vortex ring motion. 
Written as $\mathrm{S}=5$-dimensional $\mathrm{N}=2 \mathrm{nd}$ order ordinary differential circle logarithmic equation $(\mathrm{P}=1)$. According to the covariation principle, spin and revolution have the same circular logarithmic description.

Solution: According to the initial position $\mathrm{O}_{1}$ (including azimuth angle $\theta_{1}$ ), eccentricity e, elliptical orbital length and short axis $R_{(L+M)}$, constant energy characteristic mode form $\left\{M_{g 0} R_{0}\right\}$ (including mechanical parameters). Perform a logarithmic description of the elliptical orbit, velocity, and force (acceleration) of the planetary motion.

\subsubsection{The gravitational calculation of a single planet in orbit,}

Write a 5-dimensional second-order binary order partial differential equation: $\mathrm{N}=(0+1+2)$; known initial accelerationa: initial force of which: two kinds of acceleration $\left\{a_{1} a_{2}\right\}$ :

$$
\begin{gathered}
D=M_{g}\left\{a_{0}\right\}^{K(Z \pm 5 \pm 0) / t}=M_{g}\left\{R_{0}\right\}^{K(Z \pm 5 \pm 2) / t} ; \\
a=\{R\}^{K(Z \pm 5 \pm 2) / t}=\left\{r^{2}\right\}^{K(Z \pm 5 \pm 0) / t}+\left\{r^{2}\right\}^{K(Z \pm 5 \pm 1) / t}+\left\{r^{2}\right\}^{K(Z \pm 5 \pm 2) / t}=\left\{a_{1} a_{2}\right\}^{K(Z \pm 5 \pm 2) / t},
\end{gathered}
$$

(1) Single planet orbit (gravitational space)

$$
\begin{aligned}
& W(\cdot)=A r^{K(Z \pm 5 \pm 0) / t}+\mathrm{Br}^{K(Z \pm 5 \pm 1) / t}+C r^{K(Z \pm 5 \pm 2) / t} \pm \mathbf{D} \\
& =\left[A r^{K(Z \pm 5 \pm 0) / t}+B r^{K(Z \pm 5 \pm 1) / t}+C r^{K(Z \pm 5 \pm 2) / t}\right] \pm \mathbf{D} \\
& =\left[\left(1-\eta^{2}\right)^{K(Z \pm 5 \pm 0) / t}+\left(1-\eta^{2}\right)^{K(Z \pm 5 \pm 1) / t}+\left(1-\eta^{2}\right)^{K(Z \pm 5 \pm 2) / t}\right]\{0,2\} M_{g}\left\{a_{0}\right\} \\
& =\left(1-\eta^{2}\right)^{K(Z \pm 5 \pm 2) / t}\{0,2\}^{K(Z \pm 5 \pm 2) / t} M_{g}\left\{R_{0}\right\}^{K(Z \pm 5 \pm 2) / t}
\end{aligned}
$$

Solve: get acceleration, gravity:

$$
\begin{aligned}
& \left\{a_{1} a_{2}\right\}^{K(Z \pm 5 \pm 2) / t}=\left(1-\eta^{2}\right)^{K(Z \pm 5 \pm 2) / t}\left\{a_{01} a_{02}\right\}^{K(Z \pm 5 \pm 2) / t} ; \\
& \boldsymbol{F}=\left(\mathbf{1}-\boldsymbol{\eta}^{2}\right)^{K(Z \pm 5) / t}\{\mathbf{O}, 2\}^{K(Z \pm 5) / t} \boldsymbol{M}_{\boldsymbol{g}}\left\{\boldsymbol{a}_{0}\right\} \\
& =\left(\mathbf{1}-\boldsymbol{\eta}^{2}\right)^{K(Z \pm 5 \pm 2) / t} \boldsymbol{M}_{\boldsymbol{g}}\left\{\boldsymbol{R}_{\mathrm{o}}\right\}^{K(Z \pm 5 \pm 2) / t} ; \\
& \left(1-\eta^{2}\right)^{K(Z \pm 5 \pm 2) / t}=\left(1-\eta^{2}\right)^{K(Z \pm 5 \pm 0) / t}+\left(1-\eta^{2}\right)^{K(Z \pm 5 \pm 1) / t}+\left(1-\eta^{2}\right)^{K(Z \pm 5 \pm 2) / t} ;
\end{aligned}
$$

(2) Spin force around the center of mass $\{0\} \mathrm{K}(\mathrm{Z} \pm 5 \pm 2) / \mathrm{tMg}\{\mathrm{a} 01\}$

$$
\left(1-\eta^{2}\right)^{0(Z \pm 5 \pm 2) / t}=\left(1-\eta^{2}\right)^{+(Z \pm 5 \pm 2) / t}+\left(1-\eta^{2}\right)^{-(Z \pm 5 \pm 2) / t}=1
$$

(3) Spin + revolution combined movement $\{2\}^{\mathrm{K}(\mathrm{Z} \pm \mathrm{S} \pm 2) / t} \mathrm{M}_{\mathrm{g}}\left\{\mathrm{a}_{0}\right\}$

$$
\left(1-\eta^{2}\right)^{K(Z \pm 5 \pm 2) / t}=\left(1-\eta^{2}\right)^{+(Z \pm \pm \pm 2) t t}+\left(1-\eta^{2}\right)^{-(Z \pm \pm \pm 2) t t}+\left(1-\eta^{2}\right)^{0(Z \pm 5 \pm 2) / t}=2 ;
$$

(4) The force of each position $S(r, \theta)$ on the track (including the rotation of the two kinds of motion ball axes) and the linear velocity change:

$$
F(S) \approx S\left(r_{\theta}\right)=\left(1-\eta_{\theta}{ }^{2}\right)^{K(Z \pm 5 \pm 2) / t}\left\{R_{0}\right\}^{K(Z \pm 5 \pm 2) / t} ;
$$

\subsubsection{The calculation of two speeds $\{\mathbf{v} 1 \mathrm{v} 2\}$ for a single planet in orbit}

(1) 单星球平均速度计算

It belongs to the 5-dimensional first-order binary partial differential equation-circle logarithmic equation: $N=(0+1) ; D=M V_{0} ; p=1 ;$ Two speeds: 


$$
\begin{aligned}
& \mathbf{V}=\{\mathrm{R}\}^{\mathrm{K}(Z \pm 5 \pm \mathbf{1}) / \mathrm{t}}=\left\{\mathrm{r}^{2}\right\}^{\mathrm{K}(Z \pm 5 \pm 0) / \mathrm{t}}+\left\{\mathrm{r}^{2}\right\}^{\mathrm{K}(Z \pm 5 \pm \mathbf{1}) / \mathrm{t}}=\left\{\mathrm{v}_{1} \mathrm{~V}_{2}\right\}^{\mathrm{K}(Z \pm 5) / \mathrm{t}}, \\
& W(\cdot)=A v^{K(Z \pm 5 \pm \mathbf{0}) / t} \pm M_{g}\left\{R_{0}\right\}^{K(Z \pm 5 \pm \mathbf{1}) /} \\
&=\left[A r^{K(Z \pm 5 \pm \mathbf{0}) / t}+B r^{K(Z \pm 5 \pm \mathbf{1}) / t}\right] \pm M_{g}\left\{R_{0}{ }^{2}\right\}^{K(Z \pm 5 \pm \mathbf{1}) / t} \\
&=\left[\left(1-\eta^{2}\right)^{K(Z \pm 5 \pm 0) / t}+\left(1-\eta^{2}\right)^{K(Z \pm 5 \pm 1) / t}\right]\{0,2\} M_{g}\left\{R_{0}\right\}^{K(Z \pm 5 \pm \mathbf{1}) / t} \\
&=\left(1-\eta^{2}\right)^{K(Z \pm 5 \pm 1) / t}\{0,2\}^{K(Z \pm 5 \pm 1) / t} M_{g}\left\{R_{0}\right\}^{K(Z \pm 5 \pm \mathbf{1}) / t} ;
\end{aligned}
$$

Solve: get the average speed value:

$$
\left\{v_{1} v_{2}\right\}^{K(Z \pm 5 \pm \mathbf{0}) / t}=\left(1-\eta^{2}\right)^{K(Z \pm 5 \pm 2) / t}\left\{v_{01} v_{02}\right\}^{K(Z \pm 5 \pm \mathbf{0}) / t}
$$

(2) Average spin speed around the center of mass $\{0\}^{\mathrm{K}(Z \pm 5 \pm 2) / \mathrm{t}} \mathrm{M}_{\mathrm{g}}\left\{\mathrm{v}_{01}\right\}$

$$
\left(1-\eta^{2}\right)^{0(Z \pm 5 \pm 2) / t}=\left(1-\eta^{2}\right)^{+(Z \pm 5 \pm 2) / t}+\left(1-\eta^{2}\right)^{-(Z \pm 5 \pm 2) / t}=1
$$

(3) Combined average speed of spin + revolution $\{2\}^{\mathrm{K}(Z \pm s \pm 2) / t} \mathrm{Mg}_{\mathrm{g}}\left\{\mathrm{r}_{0}\right\}^{\mathrm{K}(Z \pm 5 \pm 1) / \mathrm{t}}$

$$
\left(1-\eta^{2}\right)^{K(Z \pm 5 \pm 2) / t}=\left(1-\eta^{2}\right)^{+(Z \pm 5 \pm 2) / t}+\left(1-\eta^{2}\right)^{-(Z \pm 5 \pm 2) / t}+\left(1-\eta^{2}\right)^{0(Z \pm 5 \pm 2) / t}=2
$$

\subsection{3 single planet orbit (field space) calculation}

It belongs to the 5-dimensional 0-order binary original function equation-circle logarithmic equation: $\mathrm{N}=(0+1) ; \mathrm{D}=\mathrm{MV0} ; \mathrm{p}=1$;

Let: the initial ellipse (spin + revolution) vortex orbit:

$\left\{R_{0}\right\}=\left\{r_{0}^{2}\right\}=\left\{r_{01} r_{02}\right\}=(1 / 2)\left(L_{a}+L_{b}\right)=r_{1} r_{2} ;$

form $S=5$-dimensional vortex motion orbit; $N= \pm 0 ; D=M_{g} R_{0}=M_{g}\left\{r_{0}^{2}\right\} ; p=1$; the dynamic equation time is synchronized with the calculus order value $(\mathrm{N} / \mathrm{t})$.

(1) Single-plane orbit (field) belongs to the 5-dimensional first-order binary original function equation

$$
\begin{aligned}
& W(\cdot)=A r^{K(Z \pm 5 \pm 0 \pm 1) / t}+B r^{K(Z \pm 5 \pm 0 \pm 2) / t} \pm M_{g}\left\{r_{0}^{2}\right\} \\
& =\left[\left(1-\eta^{2}\right)^{K(Z \pm 5 \pm 0) / t}+\left(1-\eta^{2}\right)^{K(Z \pm 5 \pm 1) / t}\right]\{0,2\} M_{g}\left\{r_{0}^{2}\right\} \\
& =\left(1-\eta^{2}\right)^{K(Z \pm 5 \pm 0) / t}\{0,2\}^{K(Z \pm 5 \pm 0) / t} M_{g}\left\{R_{0}\right\} ; \\
& \left\{R_{0}\right\}^{K(Z \pm 5 \pm 0) / t}=\left(1-\eta^{2}\right)^{K(Z \pm 5 \pm 0) / t}\left\{r_{01} r_{02}\right\}^{K(Z \pm 5 \pm 0) / t} ;
\end{aligned}
$$

Single-plane orbit (field) belongs to the 5-dimensional first-order binary original function equation

$$
\left(1-\eta_{0}^{2}\right)^{K(Z \pm 5 \pm 0 \pm 1) / t}=\left[\left\{\left(L_{a}-L_{b}\right)\right\} /\left\{\left(L_{a}+L_{b}\right)\right\}\right]^{K(Z \pm 5 \pm 0 \pm 1) / t} ;
$$

(2) Eccentric distance orbit $\left(1-\eta_{e}^{2}\right)^{K(Z \pm 5 \pm 0 \pm 1) / t}=[\{1-e\} /\{1+e\}]^{K(Z \pm 5 \pm 0 \pm 1) / t}$;

(3) Obtain the "eccentric vortex orbit" + "initial elliptical orbit" with the base log $\{$ RO $\}$ as the base

$$
\begin{aligned}
& \left(1-\eta^{2}\right)^{K(Z \pm 5 \pm 0) / t}=\left(1-\eta_{0}{ }^{2}\right)^{K(Z \pm 5 \pm 0) / t}+\left(1-\eta_{e}{ }^{2}\right)^{K(Z \pm 5 \pm 0) / t} ; \\
& 0 \leq\left(\eta^{2}=\eta_{0}{ }^{2}+\eta_{e}{ }^{2}\right) \leq 1
\end{aligned}
$$


Among them: (15.5.1)-(15.5.3) is a circular logarithmic factor superposition that is converted to a unified circular orbit. (The same below).

\subsection{The movement of the two-star sphere}

In the form of the two-sphere mass eigenfunction function, there are common energy boundary lines $\mathrm{W}$, E, F collectively called the energy field, and the microscopic particles are called oscillations). There are two states of parallel motion and serial motion as the superposition of the energy field space radius.

There are: energy field

$$
\mathrm{E}=\mathrm{MR}=\left(1-\eta^{\wedge} 2\right) \mathrm{MC}^{\wedge} 2=\left(1-\eta^{\wedge} 2\right) \mathrm{h} / \lambda:
$$

(1) Parallel movement "two planets parallel common centroid movement".

$$
\begin{gathered}
W=\left\{M_{g(a+b)} R\right\} \text { 或 }\left\{M_{g(a+b)} R^{2}\right\} \\
\left\{M_{g} R_{(a+b)}\right\}=(1 / 2)^{+1}\left[\left\{M_{g} R_{a}\right\}^{+1}+\left\{M_{g} R_{b}\right\}^{+1}\right]^{+1}
\end{gathered}
$$

(2) Serial motion "two-star serial" (around the common centroid movement).

$$
\begin{aligned}
& W=\left\{M_{g(a \times b)} R\right\} \text { 或 }\left\{M_{g(a \times b)} R^{2}\right\} \\
& \left\{M_{g} R_{(a \times b)}\right\}=(1 / 2)^{-1}\left[\left\{M_{g} R_{a}\right\}^{-1}+\left\{M_{g} R_{b}\right\}^{-1}\right]^{-1} \\
& \left\{M_{g(a b)} R\right\}^{K(Z \pm S \pm N \pm 2) / t}=\left\{M_{g(a b)} R^{2}\right\}^{K(Z \pm S \pm N \pm 1) / t}
\end{aligned}
$$

(3) Two-star cycle logarithm 二星球圆对数

$$
\begin{gathered}
\left(1-\eta_{0}{ }^{2}\right)=\left\{R_{(a \times b)}\right\} /\left\{R_{(a+b)}\right\} \\
=\left\{(1 / 2)^{-1}\left[\left\{\mathrm{R}^{-1}\right\}+\left\{\mathrm{R}_{\mathrm{b}}{ }^{-1}\right\}\right]^{-1}\right\} /\left\{(1 / 2)^{+1}\left[\left\{\mathrm{R}_{\mathrm{a}}{ }^{+1}\right\}+\left\{\mathrm{R}_{\mathrm{b}}{ }^{+1}\right\}\right]^{+1}\right\} ; \\
\left(1-\eta^{2}\right)^{K(Z \pm 5 \pm 0) / t}=\left(1-\eta_{0}{ }^{2}\right)^{K(Z \pm 5 \pm 0) / t}+\left(1-\eta_{e}{ }^{2}\right)^{K(Z \pm 5 \pm 0) / t} ; \\
0 \leq\left(\eta^{2}=\eta_{0}{ }^{2}+\eta_{e}{ }^{2}\right) \leq 1 ;
\end{gathered}
$$

(4) Planetary energy field W (including field space, velocity, acceleration, super acceleration)Planetary energy field $\mathrm{W}$ (including field space, velocity, acceleration, super acceleration)

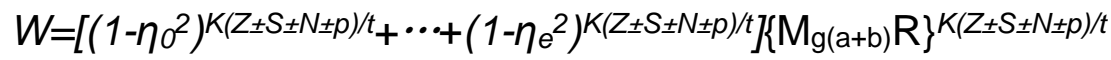

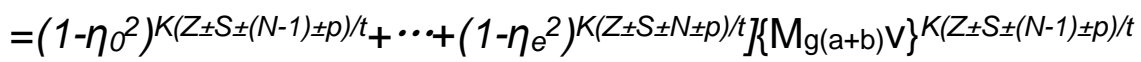

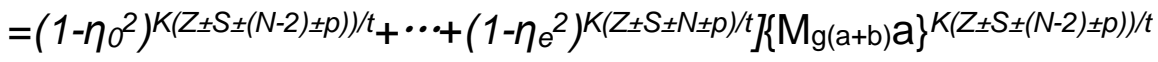

$$
\begin{aligned}
& \left(1-\eta^{2}\right)^{K(Z \pm S \pm 0 \pm p) / t}=\left(1-\eta o^{2}\right)^{K(Z \pm S \pm 1 \pm p) / t}+\left(1-\eta o^{2}\right)^{K(Z \pm S \pm 2 \pm p) / t} \\
& +\left(1-\eta o^{2}\right)^{K(Z \pm S \pm N \pm p) / t_{+}} \cdots+\left(1-\eta e^{2}\right)^{K(Z \pm S \pm N \pm p) / t}
\end{aligned}
$$

They (capable of adapting to multi-planetary energy fields) have the same form of eigenmode function, and their respective elliptical eccentric orbits (including field space, velocity, acceleration, super acceleration) have the same form. The difference is: "the center of the perfect circle" is changed to "the ellipsoid field of the binary star centroid", and the "two-sphere eccentric circular orbital vortex track" with the round logarithm $\left\{R_{0(a \times b)}\right\}$ or $\left\{R_{0(a+b)}\right\}$ ". When converting to the center of the perfect circle of the two planets, the distance of the two-sphere elliptical centroid $\eta 0$ to the centroid of the two planets (or the logarithm of the circle ne) is also added (the same below). 


\subsection{The movement of the Samsung sphere}

Applying (15.1.1)-(15.5.7) the calculation of the motion state of a single star sphere, it is applied to the calculation of the multi-planetary motion around the combined motion-fluctuation. The core is the circular logarithmic arithmetic calculation after the eigenmode function is established.

Among them, due to the equivalence and covariance of various elements, the interchangeability between elements, the quality and the orbital space are synchronic, as long as one of the known masses or tracks can be the initial condition.

Solve: (1), the combined gravity of the Samsung body:

(2), the speed of each combination of Samsung body;

(3), each combination of the Samsung body elliptical orbit (gravitational field).

Design: Radius of each planet's gravitational field $r_{A}, r_{B}, r_{C}, R_{D}$ 。 energy $\left\{M_{g(a b c)} R_{(a b c)}\right\}^{K(Z \pm S \pm N \pm 3)} ;(N=0$ or 3$)$ zero order (Original function)(N=-1)

First order (speed); $(\mathrm{N}=-2)$ Second order (acceleration, force)。 $\mathrm{K}=(+1, \pm 0,-1)$ Centripetal force $(\mathrm{k}=+1)$, centrifugal force $(k=-1)$, neutral field space $(k= \pm 0)$. In Einstein's general theory of relativity, the positive gravitational force $(k=-1)$, antigravity $(k=+1)$, and neutral field space $(k= \pm 0)$ of the surface are

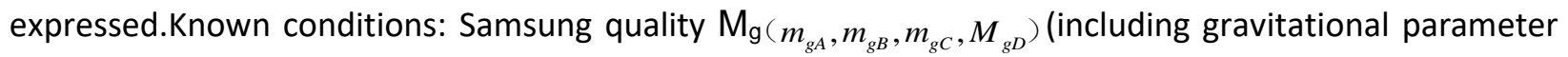
g)); $\mathbf{D}=\mathbf{D}\left(\mathbf{D}_{a}, \mathbf{D}_{a b}, \mathbf{D}_{a b c}, \mathbf{D}_{D}\right)$ Centroid point Equilibrium

Elliptical orbital long axisR $\left(r_{A(L+M)}, r_{B(L+M)}, r_{C(L+M)}, R_{D(L+M)}\right)$, Eccentricity $e_{A}, e_{B}, e_{C}, e_{D}$,

Initial position (zero order) $O_{a}, O_{b}, O_{c}, O_{D}$, Initial velocity partial differential (first order): $v_{a}, v_{b}, v_{c}, v_{D}$ Initial acceleration partial differential, force (second order) $F_{A}, F_{B}, F_{C}, F_{\mathbf{D}} ; a_{A}, a_{B}, a_{C}, a_{\mathbf{D}}$;

solution: the motion, orbital state and interaction force of the Samsung body combination (including centripetal force, centrifugal force, neutral field space or combined positive gravitational force, antigravity, neutral gravitational field space).

among them: the spherical motion energy orbit (curved surface wave) of the seven combined states of the Samsung ball is not repeated $\left(\{2\}^{3}-1=7\right)$, which is reflected as the expansion of the circular logarithmic equation with the constant form of the characteristic mode.

$$
\left(1-\eta_{[u v]}{ }^{2}\right)^{K(Z \pm 3 \pm 1 \pm p) / t}=\sum^{K(Z \pm 3 \pm 1 \pm 7)}{ }_{(i=(1 \pm p)}\left(1-\eta^{2}\right)^{K(Z \pm 3 \pm 1 \pm p) / t} ;
$$

Proof: (1), multi-sphere second-order circular logarithmic partial differential equation

$$
\begin{aligned}
& W(\cdot)=A r^{K(Z \pm S \pm \mathbf{0} \pm p) / t}+\mathrm{Br}^{K(Z \pm S \pm \mathbf{1} \pm p) / t}+\mathrm{Cr}^{K(Z \pm S \pm \mathbf{2} \pm p) / t} \pm \mathbf{D} \\
& =\left[\mathrm{A} r^{K(Z \pm S \pm \mathbf{0} \pm p) / t}+B r^{K(Z \pm S \pm \mathbf{0} \pm p) / t}+C r^{K(Z \pm S \pm \mathbf{0} \pm p) / t}\right] \\
& +\left[\mathrm{Ar} r^{K(Z \pm S \pm \mathbf{1} \pm p) / t}+B r^{K(Z \pm S \pm \mathbf{1} \pm p) / t}+C r^{K(Z \pm S \pm \mathbf{1} \pm p) / t}\right] \\
& +\left[A r^{K(Z \pm S \pm \mathbf{2} \pm p) / t}+B r^{K(Z \pm S \pm \mathbf{2} \pm p) / t}+C r^{K(Z \pm S \pm \mathbf{2} \pm p) / t}\right] \pm \mathbf{D}
\end{aligned}
$$




$$
\begin{aligned}
& =\left[\left(1-\eta^{2}\right)^{K(Z \pm 3 \pm 0 \pm p) / t}+\left(1-\eta^{2}\right)^{K(Z \pm 3 \pm \pm \pm p) / t}+\left(1-\eta^{2}\right)^{K(Z \pm 3 \pm 2 \pm p) / t}\right]\{0,2\}\left\{M_{g} R\right\}_{D} \\
& =\left[\left(1-\eta^{2}\right)^{K(( \pm \pm \pm \pm N \pm p) / t}\right]\{0,2\}\left\{M_{g} R\right\}_{D}^{K(Z \pm 3 \pm N \pm p) / t} ;
\end{aligned}
$$

(2) Circular logarithmic equation: $\mathrm{N}=0$ (zero order), $\mathrm{N}=1$ (first order, velocity), $\mathrm{N}=2$ (second order, acceleration, force)

$$
\left(1-\eta^{2}\right)^{K(Z \pm 3 \pm N \pm p) / t}=\sum_{(i=(N)}^{K(Z \pm 3)}\left(1-\eta^{2}\right)^{K(Z \pm 3 \pm N \pm p / t} ;
$$

The formulas (17.1)-(17.2) are respectively combined by the series of stars (A), (B), and (C), including a circular logarithmic factor superposition that is converted into a unified circular orbit.

8.3.1 Samsung ball "1-1 combination" $(p=1)$ serial motion surround $D=\left\{M_{g D} R_{D}\right\}$ :

$$
\begin{aligned}
& \left\{M_{g} R\right\}_{a} \rightarrow\left\{M_{g} R\right\}_{b} \rightarrow\left\{M_{g} R\right\}_{c} \rightarrow \text { 或 }\left\{M_{g} R\right\}_{a} \rightarrow\left\{M_{g} R\right\}_{c} \rightarrow\left\{M_{g} R\right\}_{b} \rightarrow ; \\
& \left\{M_{g} R\right\}_{b} \rightarrow\left\{M_{g} R\right\}_{a} \rightarrow\left\{M_{g} R\right\}_{c} \rightarrow \text { 或 }\left\{M_{g} R\right\}_{b} \rightarrow\left\{M_{g} R\right\}_{c} \rightarrow\left\{M_{g} R\right\}_{a} \rightarrow ; \\
& \left\{M_{g} R\right\}_{c} \rightarrow\left\{M_{g} R\right\}_{a} \rightarrow\left\{M_{g} R\right\}_{b} \rightarrow \text { 或 }\left\{M_{g} R\right\}_{c} \rightarrow\left\{M_{g} R\right\}_{b} \rightarrow\left\{M_{g} R\right\}_{a} \rightarrow ;
\end{aligned}
$$

Energy circle logarithm $\left\{M_{g D} R_{D}\right\}$ :

$$
\begin{aligned}
& \left(1-\eta_{A}{ }^{2}\right)^{K(Z \pm 3 \pm N \pm 1) / t}=\left\{M_{a} R_{a}\right\} /\left\{M_{g D} R_{D}\right\}=\left\{M_{a}\right\} /\left\{M_{D}\right\}=\left\{R_{a}\right\} /\left\{R_{D}\right\} ; \\
& \left(1-\eta_{B}{ }^{2}\right)^{K(Z \pm 3 \pm N \pm 1) / t}=\left\{M_{b} R_{b}\right\} /\left\{M_{D} R_{D}\right\}=\left\{M_{b}\right\} /\left\{M_{D}\right\}=\left\{R_{b}\right\} /\left\{R_{D}\right\} ; \\
& \left(1-\eta_{C}{ }^{2}\right)^{K(Z \pm 3 \pm N \pm 1) / t}=\left\{M_{c} R_{c}\right\} /\left\{M_{D} R_{D}\right\}=\left\{R_{c}\right\} /\left\{R_{D}\right\}=\left\{R_{c}\right\} /\left\{R_{D}\right\}
\end{aligned}
$$

Unit circle logarithm:

$$
\left(1-\eta_{H}{ }^{2}\right)=\sum_{(i=(1 \pm p)}^{K(Z \pm 3 \pm 1 \pm 7)}\left\{M_{i} R_{i}\right\} /\left\{M_{D} R_{D}\right\}=\left(1-\eta_{h 1}{ }^{2}\right)+\left(1-\eta_{h 2}{ }^{2}\right)+\left(1-\eta_{h 3}{ }^{2}\right)=1
$$

8.3.2 Samsung ball " $2-2$ combination" $(p=2)$ parallel surround with a serial $\rightarrow$ surround $D=\left\{M_{g D} R_{D}\right\}$

$$
\begin{aligned}
& \left(1-\eta_{A(2)}{ }^{2}\right)^{K(Z \pm 3 \pm N \pm 2) / t}=\left\{M_{a b} R_{a b}+M_{c} R_{c}\right\} /\left\{M_{D} R_{D}\right\}=\left\{M_{a b}\right\} /\left\{M_{D}\right\}=\left\{R_{a b}\right\} /\left\{R_{D}\right\} ; \\
& \left(1-\eta_{B(2)}{ }^{2}\right)^{K(Z \pm 3 \pm N \pm 2) / t}=\left\{M_{b c} R_{b c}+M_{a} R_{a}\right\} /\left\{M_{D} R_{D}\right\}=\left\{M_{b c}\right\} /\left\{M_{D}\right\}=\left\{R_{b c}\right\} /\left\{R_{D}\right\} ; \\
& \left(1-\eta_{C(2)}{ }^{2}\right)^{K(Z \pm 3 \pm N \pm 2) / t}=\left\{M_{c a} R_{c a}+M_{b} R_{b}\right\} /\left\{M_{D} R_{D}\right\}=\left\{R_{c a}\right\} /\left\{R_{D}\right\}=\left\{R_{c a}\right\} /\left\{R_{D}\right\} ;
\end{aligned}
$$

Energy circle logarithm:

$$
\begin{aligned}
& \left(1-\eta_{A(2)}{ }^{2}\right)^{K(Z \pm 3 \pm N \pm 2) / t}=\left\{M_{a b} R_{a b}+M_{c} R_{c}\right\} /\left\{M_{D} R_{D}\right\}=\left\{M_{a b}\right\} /\left\{M_{D}\right\}=\left\{R_{a b}\right\} /\left\{R_{D}\right\} ; \\
& \left(1-\eta_{B(2)}{ }^{2}\right)^{K(Z \pm 3 \pm N \pm 2) / t}=\left\{M_{b c} R_{b c}+M_{a} R_{a}\right\} /\left\{M_{D} R_{D}\right\}=\left\{M_{b c}\right\} /\left\{M_{D}\right\}=\left\{R_{b c}\right\} /\left\{R_{D}\right\} ; \\
& \left(1-\eta_{C(2)}{ }^{2}\right)^{K(Z \pm 3 \pm N \pm 2) / t}=\left\{M_{c a} R_{c a}+M_{b} R_{b}\right\} /\left\{M_{D} R_{D}\right\}=\left\{R_{c a}\right\} /\left\{R_{D}\right\}=\left\{R_{c a}\right\} /\left\{R_{D}\right\} ;
\end{aligned}
$$

Unit circle logarithm:

$$
\left(1-\eta_{H}{ }^{2}\right)=\sum_{(i=[1 \pm p)}^{K(Z \pm 3 \pm 1 \pm 7)}\left\{M_{i} R_{i}+M_{j} R_{j}\right\} /\left\{M_{D} R_{D}\right\}=\left(1-\eta_{h 1}{ }^{2}\right)+\left(1-\eta_{h 2}{ }^{2}\right)+\left(1-\eta_{h 3}{ }^{2}\right)=1
$$

8.3.3 Samsung ball "0-3 combination" ( $p=0,3)$ parallel surround or serial surround

$$
\left[\left\{M_{g} R\right\}_{a}+\left\{M_{g} R\right\}_{b}+\left\{M_{g} R\right\}_{c}\right] \rightarrow ; D=\left\{\mathrm{MgDR}_{\mathrm{D}}\right\}=\left\{\mathrm{MgABCR}_{\mathrm{ABC}}\right\}:
$$

Energy circle logarithm:

$$
\left(1-\eta_{(3)}{ }^{2}\right)^{K(Z \pm 3 \pm N \pm 3) / t}=\left\{M_{a} R_{a}\right\} /\left\{M_{D} R_{D}\right\}=\left\{M_{a}\right\} /\left\{M_{D}\right\}=\left\{R_{a}\right\} /\left\{R_{D}\right\} ;
$$


Unit circle logarithm:

$$
\begin{aligned}
& \left(1-\eta_{(3)}{ }^{2}\right)^{K(Z \pm S \pm N \pm p) / t}=\sum_{(i=h)} K(Z \pm S \pm N \pm p)\left\{M_{g(h i)} R_{(h i)}\right\} /\left\{M_{g(A B C)} R_{(A B C)}\right\} \\
& =\left(1-\eta_{A}{ }^{2}\right)^{K(Z \pm S \pm N \pm p) / t}+\left(1-\eta_{B}{ }^{2}\right)^{K(Z \pm S \pm N \pm p) / t}+\left(1-\eta_{C}{ }^{2}\right)^{K(Z \pm S \pm N \pm p) / t} ;
\end{aligned}
$$

\subsection{Samsung ball total isomorphic logarithm (energy orbit, gravitational field)}

$$
\left(1-\eta_{D}{ }^{2}\right)^{K(Z \pm 3 \pm N \pm p) / t}=\left(1-\eta_{A}^{2}\right)^{K(Z \pm 3 \pm N \pm p) / t}+\left(1-\eta_{B}^{2}\right)^{K(Z \pm 3 \pm N \pm p) / t}+\left(1-\eta_{C}^{2}\right)^{K(Z \pm 3 \pm N p) / t}
$$

Unit circle logarithm:

$$
\begin{aligned}
& \left(1-\eta_{H}{ }^{2}\right)^{K(Z \pm 3 \pm 0 \pm p) / t}=\left[\left\{M_{g(a)} R_{(a)}\right\}+\left\{M_{g b)} R_{(b)}\right\}+\left\{M_{g(c)} R_{(c)}\right\}\right] /\left\{M_{g(a b c)} R_{(a b c)}\right\} \\
& =\left(1-\eta_{h 1}{ }^{2}\right)^{K(Z \pm 3 \pm N \pm 1) / t}+\left(1-\eta_{h 2}{ }^{2}\right)^{K(Z \pm 3 \pm N \pm 1) / t}+\left(1-\eta_{h 3}{ }^{2}\right)^{K(Z \pm 3 \pm N \pm 1) / t} ;
\end{aligned}
$$

Circular logarithmic properties: $K=+1$ (central force), $K=-1$ (centrifugal force), $K= \pm 0$ (gravitational field space),

$$
\left(1-\eta_{(A B C)}^{2}\right)^{K(Z \pm 3 \pm N \pm p) / t}=\left(1-\eta^{2}\right)^{+(Z \pm 3 \pm N \pm 1) / t}+\left(1-\eta^{2}\right)^{0(Z \pm 3 \pm N \pm 2) / t}+\left(1-\eta^{2}\right)^{-(Z \pm 3 \pm N \pm 3) / t}
$$

By the same token, the above circular logarithm algorithm can also adapt to any finite $(\mathrm{Z} \pm \mathrm{S} \pm \mathrm{N} \pm \mathrm{P}) / \mathrm{t}$ (galaxies-quantity particle-cluster) superma in the fields of macrocosm, microscopic quantum, mesoscopic molecules-cells, etc. The energy of $\mathrm{N}(\mathrm{N} \geqslant 3)$ order and the superhigh-order circular logarithmic partial differential equation of field $\mathrm{K}(\mathrm{Z} \pm \mathrm{S} \pm \mathrm{N}) / \mathrm{t}$ (this article is omitted). By the same token, the above circular logarithm algorithm can also adapt to any finite $\mathrm{Z} \pm \mathrm{S} \pm \mathrm{N} \pm \mathrm{P}) / \mathrm{t}$

(galaxies-quantity particle-cluster) superma in the fields of macrocosm, microscopic quantum, mesoscopic molecules-cells, etc. The energy of $\mathrm{N}(\mathrm{N} \geq 3)$ order and the superhigh-order circular logarithmic partial differential equation of field $\mathrm{K}(\mathrm{Z} \pm \mathrm{S} \pm \mathrm{N}) / \mathrm{t}$ (this article is omitted).

\section{The unity of the circular logarithm and the gauge field and the natural force}

In 1954, Yang Zhenning-Mills proposed the famous "normative field" theory. The gauge field is a material field associated with the invariance of localized normative transformation of physical laws.

In 1961, Grasho proposed the $\mathrm{SU}(2) \times \mathrm{U}(1)$ group of four generators (photons, W+ particles, W-particles, and neutral flow vector bosons).

In 1967, Weinberg and Salam developed a unified theory of weak electricity for these four "generating elements". It is called quantum taste dynamics (QED). Since the 1970s, Grasho and Qiaoqi have chosen the SU(3) group to establish a unified theory describing the three strong interactions of weak, strong and electromagnetic, called quantum chromodynamics (QCD). It is hoped that the gravitational interactions will also be unified by the gauge field. At this time, scientists represented by Einstein know that gravitational interaction is also a normative field, and it is not known how gravitation is related to the other three interactions.

The Western European Nuclear Research Center has experimented with positive and negative proton collisions and annihilation and unexplained increases of "super energy" and various unknown radiation particles; physicists have also discovered gravitational waves, Mayorana Rice, low-temperature-normalhigh temperature superconductors, thermonuclear fission, and cold nuclear fusion reflect the interaction 
of four basic particles with light (including neutral active particles) and temperature. It presents the interaction characteristics of "symmetry and asymmetry, uniformity and non-uniformity, continuous and discontinuous, random and regular" in which various particles have reciprocal properties. It is suspected that there is a possibility of uniformity.

In 1905, Yang Zhenning-Mills gauge field = Dirac mechanical equation + Maxwell's electromagnetic force equation + multi-element multiplication of the gauge field:

$$
\begin{aligned}
& W(X)=L\left[\psi(x), A_{\mu}(x)\right] \\
& =\psi^{k}\left(\gamma_{\mu}\left(d / d x_{\mu}\right)+m\right)+\psi^{k}(1 / 4) F_{\mu \nu} F_{\mu \nu}+L e \psi^{k} \gamma_{\mu} \psi A_{\mu} ;
\end{aligned}
$$

The various quality elements of the formula (22.1) performance gauge field are closely related to the asymmetric topological space. The core is that the six "generating elements" are composed of "quality $\{M\}$-space $\left\{R^{2}\right\}$ " of "infinite multi-element quality" and form a sixth-order partial calculus equation with original function (zero order), velocity (first order), acceleration (Second order), super acceleration (third order or more) infinite program expansion, and is also a combination of any limited topological combination between the center point and the boundary line.

There are: Abel gauge field theory $\left\{\Psi_{\mathrm{uv}}(\mathrm{x})\right\} \bullet\left\{\mathrm{A}_{\mu \mathrm{v}}(\mathrm{x})\right\}=1$;

$$
\{M R\}^{Z / t}=L\left\{\psi_{u v}(x), A_{\mu v}(x)\right\}^{Z / t} \in\left\{I e \psi_{q} Q \gamma_{\mu} \psi_{q} \ldots \mathbf{B}_{\mathrm{uv}}\right\}^{Z / t}=\{0 \text { or } 1\}^{Z / t},
$$

There are: non-Abelian gauge field theory $\left.\left\{\Psi_{\mathrm{uv}}(\mathrm{x})\right\} \cdot\{\} \mathrm{A}_{\mu \mathrm{v}}(\mathrm{x})\right\} \neq 1$;

$$
\{M R\}^{Z / t}=L\left\{\psi_{u v}(x), A_{\mu v}(x)\right\}^{Z / t} \in\left\{I e \psi_{q} Q \gamma_{\mu} \psi_{q} \ldots \mathbf{B}_{\mathbf{u v}}\right\}^{Z / t}=\left\{\begin{array}{ll}
0 & \text { to } 1
\end{array}\right\}^{Z / t},
$$

where: Buv represents the various interactions of the gauge fielda set of surface functions formed by $\psi A_{\mu}$, $L\left\{e \psi^{k} \gamma_{\mu} \psi_{u} A_{\mu}\right\} \in L\left\{e \psi_{q} Q \gamma_{\mu} \psi_{u v} B_{u v}\right\}=\left\{R_{u v}{ }^{2}{ }^{z / t}\right.$.represents the dynamic equations of various mass masses $\{M\}$, field surface space $\left\{R_{\mathrm{uv}}\right\}$, and mechanical parameters.

\subsection{The specification field is unified by the logarithm of the circle}

Let: photon (including neutral active particle), electron, gravity, hadron, weak, and thermal force are six "generating elements $(\mathrm{N}=6)$ " to form a sixth-order partial calculus equation, each generating element (The order has the infinite element mass $\left\{\mid \mathrm{e} \psi_{q} \mathrm{Q}_{{ }_{\mu}} \psi_{\mathrm{q}} \cdots\right\}$ and the mechanical parameter $\left\{\mathrm{B}_{\mu v}\right\}$, and its interaction produces entanglement. (Note: Neutral active particle means that it can interact with other particles, and vice versa.)

Here, the sixth-order partial calculus equation consists of "generating element" and "elemental mass (including various combinations of infinity $\pm p)$ ", which constitute a $Z / t=K(Z \pm S \pm N \pm p) / t$ power function.

wherein, the order of the six "generating elements" is not repeated $\pm N=\{0,1,2,3,4,5,6\}$, from "0-0 combination, 1-1 combination, 2-2 combination, 3-3 combination, 4-4 combination, 5-5 combination, 6-6 combination" form, the sixth-order (dimension) regularization combination coefficient has $\{2\}^{6}=64$ combinations, power function $\mathrm{K}=(+1,0,-1)$ reflects the nature of the positive, medium and inverse functions, and facilitates the uniform description of the logarithm of the circle.

According to the equivalent substitution theorems $\left\{M R^{2}\right\},\{M\},\left\{R^{2}\right\} 、\left\{X^{2}\right\}$ are equivalently replaceable, the gauge field is written as $\{R\}$, and mathematically written as $\{X\}$. 
Design: Yang Zhenning-Mills non-Abelian normative field is rewritten into six sixth-order partial calculus dynamic equations composed of six "generating elements".

$$
\begin{aligned}
& \left\{M R^{2}\right\}^{K(Z \pm S \pm N \pm p) / t}=A r^{K(Z \pm S \pm \mathbf{0} \pm p) / t}+B r^{K(Z \pm S \pm \mathbf{1} \pm p) / t}+C r^{K(Z \pm S \pm \mathbf{2} \pm p) / t} \\
& +D r^{K(Z \pm S \pm \mathbf{3} \pm p) / t}+E r^{K(Z \pm S \pm \mathbf{4} \pm p) / t}+F r^{K(Z \pm S \pm 5 \pm p) / t}+\ldots+S r^{K(Z \pm S \pm \mathbf{6} \pm p) / t}+\mathbf{D} ;
\end{aligned}
$$

where: $A, B, C, \cdots S$ are integer coefficients of the calculus equation, and the regularized combination coefficients of the implicit sub-items. The mass of the unknown element $\{r\}$ (including the mechanical parameters); the known element boundary condition $D . D=\left\{D_{0}\right\}^{K(Z \pm S \pm 6 \pm p) / t}$; establish a logarithmic equation.

$$
\begin{gathered}
W=\left(1-\eta^{2}\right)^{K(Z \pm S \pm 6 \pm p) / t}\{0,2\}^{K(Z \pm S \pm 6 \pm p) / t}\left\{D_{0}\right\}^{K(Z \pm S \pm 6 \pm p) / t} \\
\left(1-\eta_{C}{ }^{2}\right)^{0(Z \pm S \pm N \pm p) / t}=\left\{{ }^{K( \pm S \pm N)} \sqrt{ } \mathbf{D} /\left\{D_{0}\right\}^{K(Z \pm S \pm 6 \pm p) / t}\right.
\end{gathered}
$$

\subsection{The third item: the normative field}

The mass combination mechanical parameters of the various quantities of particle interactions, the $\beta_{\mathrm{uv}}$ series; the gauge fielnctiond is a neutral fu. 各种量粒子相互作用的质量组合力学参数 $\beta_{\mathrm{uv}}$ 系列; 规范 场属于中性函数。

$$
\begin{aligned}
& \{x\}^{0 Z / t}=\left\{\operatorname{Iew}_{q} Q \gamma_{\mu} \psi_{q} B_{\mu}\right\}^{ \pm 0} \mathbf{B}_{\mathbf{u v}} \\
& =\left(1-\eta_{\mathrm{C}}{ }^{2}\right)^{\mathrm{O}(Z \pm S \pm N \pm p) / t}\{0,2\}^{K(Z \pm S \pm N \pm p) / t}\left\{W_{c}\left(x_{0}\right)\right\}^{K(Z \pm S \pm N \pm p) / t}
\end{aligned}
$$

(1), "1-1 combination" $\mathrm{N}= \pm 1$; a "generating element" of the combined normative field $\left\{\mathrm{R}_{1}\right\}^{\mathrm{z} / \mathrm{t}}$, mechanical parameter $\boldsymbol{\alpha}_{\mathrm{w}}$ series, "generating element" = 6 separate forms; " 1

$$
\begin{aligned}
& \left\{x^{1}\right\}^{Z / t}=\left\{\operatorname{Ie}_{q} Q \gamma_{\mu} \psi_{q} B_{\mu}\right\} \boldsymbol{\alpha}_{\mathbf{w}} \\
& =\left(1-\eta_{1}{ }^{2}\right)^{K(Z \pm S \pm 1 \pm p) / t}\{0,2\}^{K(Z \pm S \pm N \pm p) / t}\left\{W_{c 1}\left(x_{0}\right)\right\}^{K(Z \pm S \pm N \pm p) / t} ;
\end{aligned}
$$

(2) "2-2 combination" $\mathrm{N}= \pm 2$ : gauge field of the multiplicative combination of two "generating element" interactions, mechanical parameter $\boldsymbol{\beta}_{\boldsymbol{w}}$ series, "generating element" = 15 combinations;

$$
\begin{aligned}
& \left\{x^{2}\right\}^{Z / t}=\left\{\operatorname{Ie\psi }_{q} Q \gamma_{\mu} \psi_{q} B_{\mu}\right\} \boldsymbol{\beta}_{\mathrm{w}} \\
= & \left(1-\eta_{2}{ }^{2}\right)^{K(Z \pm S \pm N \pm p) / t}\{0,2\}^{K(Z \pm S \pm N \pm p) / t}\left\{W_{c 2}\left(x_{0}\right)\right\}^{K(Z \pm S \pm N \pm p) / t} ;
\end{aligned}
$$

(3) "3-3 combination" $\mathrm{N}= \pm 3$ : the gauge field of the multiplicative combination of three "generating element" interactions, the mechanical parameter $\boldsymbol{\gamma}_{w}$ series "generating element" $=20$ combinations;

$$
\begin{aligned}
& \left\{x^{3}\right\}^{Z / t}=\left\{I e \psi_{q} Q \gamma_{\mu} \psi_{q} B_{\mu}\right\} \gamma_{w} \\
& =\left(1-\eta_{3}{ }^{2}\right)^{K(Z \pm S \pm N \pm p) / t}\{0,2\}^{K(Z \pm S) t}\left\{W_{c 3}\left(x_{0}\right)\right\}^{K(Z \pm S \pm N \pm p) / t} ;
\end{aligned}
$$

(4) "4-4 combination" $\mathrm{N}= \pm 4$ : gauge field mechanical parameters of the four-generation combination of four "generating element" interactions $\boldsymbol{\delta}_{w}$ series "generating elements" = 15 combinations;

$$
\begin{aligned}
& \left\{x^{4}\right\}^{Z / t}=\left\{I e \psi_{q} Q \gamma_{\mu} \psi_{q} B_{\mu}\right\} \boldsymbol{\delta}_{\mathbf{w}} \\
= & \left(1-\eta_{4}{ }^{2}\right)^{K(Z \pm S \pm N \pm p) / t}\{0,2\}^{K(Z \pm S) t}\left\{W_{c 4}\left(x_{0}\right)\right\}^{K(Z \pm S \pm N \pm p) / t} ;
\end{aligned}
$$


(5)、" $5-5$ combination" $N= \pm 5$ : gauge field mechanical parameters of the multiplicative combination of five "generating element" interactions $\zeta_{w}$ series "generating elements" = 6 separate forms;

$$
\begin{aligned}
& \left\{x^{5}\right\}^{Z / t}=\left\{\operatorname{Ie} \psi_{q} Q \gamma_{\mu} \psi_{q} B_{\mu}\right\} \zeta_{\mathrm{w}} \\
& =\left(1-\eta_{5}{ }^{2}\right)^{K(Z \pm S \pm N \pm p) / t}\{0,2\}^{K(Z \pm S \pm N \pm p) / t}\left\{W_{c 5}\left(x_{0}\right)\right\}^{K(Z \pm S \pm N \pm p) / t} ;
\end{aligned}
$$

(6) "6-6 combination $N= \pm 6, \pm 0$ ": the gauge field mechanical parameter $\lambda_{w}$ series "generating element" of the multiplicative combination of six "generating element" interactions $=1$ multiplicative combination form;

$$
\begin{aligned}
& \left\{x^{5}\right\}^{Z / t}=\left\{\operatorname{Iew}_{q} Q \gamma_{\mu} \psi_{q} B_{\mu}\right\} \zeta_{\mathbf{w}} \\
& =\left(1-\eta_{5}{ }^{2}\right)^{K(Z \pm S \pm N \pm p) / t}\{0,2\}^{K(Z \pm S \pm N \pm p) / t}\left\{W_{c 5}\left(x_{0}\right)\right\}^{K(Z \pm S \pm N \pm p) / t} ;
\end{aligned}
$$

(7) Normal, medium and negative nature norm field and circular logarithm

$$
\left(1-\eta_{C}\right)^{K(Z \pm S \pm N \pm p) / t}=\sum_{(i=6)}^{K(Z \pm S \pm N \pm p)}\left(1-\eta_{i}^{2}\right)^{K(Z \pm S \pm N \pm p) / t} ;
$$

The formulas (24.1)-(24.7) belong to the neutral gauge field, and there are three states of positive, medium and negative $\{2\}^{6}-1=64$ reciprocal theorems. That is to say, Yang Zhenning's non-Abelian normative field formula field formula can be decomposed into the first mechanical non-Abelian gauge field and the second electromagnetic force reverse non-Abelian gauge field.

\subsection{The first term: the norm field of the Dirac mechanical equation $\{r 3, r 6, r 9\} Z / t=\{r 3\} Z / t$;}

The mechanical parameter $\mathbf{G}_{\mathbf{N}}$ series; belongs to the positive boson function. $K=(+1)$.

$$
\begin{aligned}
& \left\{x^{3}\right\}^{+Z / t}=\psi^{k}\left(\gamma_{\mu}\left(d / d x_{\mu}\right)+m\right)=\left\{\operatorname{Ie}_{q} Q \gamma_{\mu} \psi_{g} B_{\mu}\right\}^{+1} \\
& =\left(1-\eta_{A}{ }^{2}\right)^{+(Z \pm S \pm N \pm p) / t}\{0,2\}^{K(Z \pm S \pm N \pm p) / t}\left\{W_{A}\left(x_{0}\right)\right\}^{K(Z \pm S \pm N \pm p) / t} ;
\end{aligned}
$$

\subsection{The second term:}

the norm field of Maxwell's electromagnetic equation $\{\mathrm{r} 2, \mathrm{r} 4 \mathrm{ZZ} / \mathrm{t}=\{\mathrm{R} 4\} \mathrm{Z} / \mathrm{t} \cdot$ The mechanical parameter $\mathrm{KN}$ series; belongs to the inverse Fermion function. $K=(-1) \pm S \pm N \pm p) / t\{W B(x 0)\} K(Z \pm S \pm N \pm p) / t$;

$$
\begin{aligned}
& E_{\Omega}=\left(1-\eta_{[u v]}{ }^{2}\right)^{K(Z \pm S \pm N \pm p) / t}\left\{D_{0}\right\}^{K(Z \pm S \pm N \pm p) / t} ; \\
& 0 \leq\left(1-\eta_{[u v]}{ }^{2}\right)^{K(Z \pm S \pm N \pm p) / t} \leq 1 ; \\
& \left(1-\eta_{[u v]}{ }^{K(Z / t)}=\left(1-\eta_{A}{ }^{2}\right)^{K(Z / t)}+\left(1-\eta_{B}{ }^{2}\right)^{K(Z / t)}+\left(1-\eta_{C}{ }^{2}\right)^{K(Z / t)}=\left\{^{K( \pm S \pm N)} \sqrt{ } /\left\{D_{0}\right\}^{K(Z \pm S \pm 6 \pm p) / t}\right.\right. \\
& \left\{W_{[u v]}\left(x_{0}\right)\right\}=W_{A}\left(x_{0}\right)+W_{B}\left(x_{0}\right)+W_{C}\left(x_{0}\right)=\left\{^{K( \pm S \pm N)} \sqrt{ }\right\}^{K(Z \pm S \pm 6 \pm p) / t} \\
& \left\{W_{[u v]}\left(x_{0}\right)\right\} \in\left\{W_{C}\left(x_{0}\right),\left(W_{A}\left(x_{0}\right), W_{B}\left(x_{0)}\right)\right\} ;\right.
\end{aligned}
$$

The above-mentioned gauge fields have the same change rule, except that the interaction produces a series of "different mechanical parameters $\left(\mathbf{G}_{N}, \mathbf{G}_{N}, \boldsymbol{\alpha}_{w}, \boldsymbol{\beta}_{w}, \mathbf{Y}_{w}, \boldsymbol{\delta}_{w}, \boldsymbol{\zeta}_{w}, \boldsymbol{\lambda}_{w}\right)$ (including various combinations of infinite mass elements in the series), The electromagnetic log field-weak nuclear force field-strong nuclear force field-photon force field-gravitational field-thermal field "six generators" can be selfconsistent to realize a unified circular logarithmic equation. Realize "topological superposition without specific quality content, accurately solve in the range of [0 1/2 1]". among them: 
(1) The unit log logarithm corresponds to the characteristic mode gauge field: the mean value of the [0 or 1] function of the infinite particle at the center zero or boundary $\left\{D_{\Omega}\right\}^{K(Z \pm S \pm N \pm p) / t}$ :

$$
\begin{aligned}
& \left(1-\eta_{[u v]}\right)^{K(Z \pm S \pm N \pm p) / t}=\left\{I e+\psi_{q}{ }^{k}+Q+\gamma_{\mu+} \psi_{q}+\ldots \mathbf{B}_{\mathrm{uv}}\right\} / \Sigma\left\{I e+\psi_{q}{ }^{k}+Q+\gamma_{\mu}+\psi_{q}+\ldots \mathbf{B}_{\mathrm{uv}}\right\} \\
& =\{0 \text { or } 1\}^{K(Z \pm S \pm N \pm p) /} ;
\end{aligned}
$$

(2) Isomorphic circle logarithm corresponding topological gauge field: topological composition of any finite element calculation unit $\left(1-\eta_{\Omega}^{2}\right)^{K(Z \pm S \pm N \pm p) / t}$

$$
\begin{aligned}
& \left(1-\eta_{[u v]}{ }^{2}\right)^{K(Z \pm S \pm N \pm p) / t}=\left\{I e \psi_{q}{ }^{k} Q \gamma_{\mu} \psi_{q} \ldots \mathbf{B}_{\mathrm{uv}}\right\}^{-1} /\left\{I e \psi_{q}{ }^{k} \mathrm{Q} \gamma_{\mu} \psi_{q} \ldots \mathbf{B}_{\mathrm{uv}}\right\}^{+1} \\
= & \left\{\left(1-\eta_{A}{ }^{2}\right)^{+1}+\left(1-\eta_{B}{ }^{2}\right)^{-1}+\left(1-\eta_{C}{ }^{2}\right)^{ \pm 0}\right. \\
= & \left\{\begin{array}{lll}
0 & 1 & 1
\end{array}\right\}^{K(Z \pm S \pm N \pm p) / t}
\end{aligned}
$$

(3), The logarithmic zero point (phase change point, center point):(a) Adapted to: Fermion (hadron, weak, gravitational, electromagnetic), belonging to the entangled state.

$$
\left(1-\eta_{[u v]}^{2}\right)^{K(Z / t)}=\{0,(1 / 2), 1\}^{K(Z \pm S \pm N-p) / t} S, ;
$$

(b)、Adapted to: bosons (photonthermodynamics, neutral particles) belong to the discrete state.

$$
\left(1-\eta_{[u v]}\right)^{K(Z / t)}=\{0,(1 / 2+1 / 2),(1 / 3+2 / 3), 1\}^{K(Z \pm S \pm N+p) / t} ;
$$

Special,

(1) Normalized field-quantity particles In the combination of regularization coefficients of any finite topology, the internal combination can be defects, vacancies, sparse, irregular distribution, without affecting the combination coefficient and the mean value of the function of all particle integrity, The variable light speed) exists of the characteristic mode function.

(2) Combining integers and power function factors based on infinite programs is a unitary expansion. Their zero point (limit) has a stable infinite "zero error" presence.

(3) The series of mechanical parameters are hidden in the eigenmode function of each sub-item of infinite program and arbitrary finite topology combination. Does not appear in the logarithmic equation.

(4) Each of the forward boson specification field and the reverse Fermion specification field, and there are three normal, medium and negative specification fields of the respective sub-items. Therefore, the logarithm of the circle contains the existing classical and modern physical formulas, which are all special cases or partial descriptions of the above formulas.

\section{Verification: Mathematical simulation of the relationship between the cosmological equation and the logarithm of the circle}

The space $\{\Omega\}$ has two states of spin and public rotation. They can exist independently or in combination, and can exist in one dimension of eleven-dimensional space and time. The mathematical description has five-dimensional vortex space and six-dimensional Karaby-Qiu space. Each space (group, cluster, galaxies, planet, and particle) has the symmetry and asymmetry of the reciprocal theorem. The logarithm of the circle proves that under the entanglement of six natural forces (light, gravity, electromagnetic force, hadron force, electromagnetic force, temperature), each element is interdependent and mutually restrictive. 


\subsection{Calculation examples of parallel/serial equations for the 11-dimensional equation of the universe $\{\Omega\}$}

Here the numbers are used with five (neutral inactive particles) with the smallest natural number $(1,2,3,4,5)$ and (gravitational field - weak nuclear field - photon force field - thermal field - strong nuclear field - The electromagnetic field) uses six prime numbers $(3,3,5,7,11,13)$ to simulate the interpretation and calculation of the cosmological equation, the evolution of the universe, the non-conservation of parity, and the super-energy of vacuum excitation.

Let: the universe $\{\Omega\}$ constitutes an arbitrary finite 11-dimensional equation, consisting of two parallel analog algebraic integer equations: one of the fifth-order equations is a discrete symmetric calculation; the first-order sixth-order equation is an entangled asymmetry calculation. The parallel/serial calculation of the eleven-dimensional equation is superimposed on the above two equations.

[Analog data A]: Known condition: boundary condition D5=243; another key tells: variable is five natural numbers; discrete one-element fifth-order equation;

[Analog data B]: Known condition: boundary $D_{6}=45045$; another key tells: the variable is six prime numbers. Entangled first-order sixth-order equation,

\section{[Analog data $C]=[$ Analog data $A]+[$ Analog data $B]=D_{5}+D_{6}=243+45045=45188$;}

Its power function: $Z=K(Z \pm \Omega \pm 11)=K(Z \pm S \pm 5)+K(Z \pm S \pm 6)$ respectively form a logarithmic equation to directly calculate the values of each universe element.

\subsection{Digital simulation of the universe equation $\{\Omega\}$}

As a group and a cluster unit, the universe has symmetry and entanglement, respectively, and performs a vortex motion or evolution of the median and inverse properties. The composition of the whole universe and individual star clusters, galaxies, and stars, with a neutral symmetry reciprocal $\mathrm{K}=( \pm 0$ ) (energy conservation). Where: $\left[D_{0 \Omega}\right]^{\mathrm{K}(\mathrm{Z} \pm \Omega \pm N) / t}$ is a known boundary condition.

For example, the initial state of observation, pre-test (a priori value, target mean value) and postobservation (posterior value, average value) are symmetrical. This part has a description of the reciprocal $\mathrm{K}=( \pm 0)$ (neutral) of the symmetry of the whole symmetry, such as neutral light and energy conservation. It belongs to discrete quantum computing.

For example, after observation, after test (posterior value, actual average value), the result is asymmetrical, and the reciprocal $\mathrm{K}=(+1,-1)$ (positive, negative) description with asymmetry. It belongs to entangled quantum computing.

There are: the logarithm of the universe $\Omega$ :

$$
\begin{aligned}
& \left.\left(1-\eta_{\Omega}^{2}\right)^{K(Z \pm \Omega \pm N) / t}=\left[{ }^{K(11 \pm N)} \sqrt{ } D_{\Omega}\right]^{K(Z \pm \Omega \pm N \pm 11) / t} / D_{024}\right]^{K(Z \pm S \pm N \pm 11) / t} \\
& =\left[{ }^{K(11 \pm N)} \sqrt{ } D_{\Omega}\right]^{K(Z \pm \Omega \pm N \pm 11) / t} /\left[D_{0 \Omega}\right]^{K(Z \pm \Omega \pm N \pm 11) / t}=(0 \text { to } 1)
\end{aligned}
$$

$\operatorname{Cosmic} \Omega$ circle logarithmic equation 


$$
\begin{aligned}
& \left.W_{\Omega}\{R\}=\left(1-\eta_{\Omega}^{2}\right)^{K(Z \pm \Omega \pm N \pm 11) / t}\left\{D_{0 \Omega}\right\}\right]^{K(Z \pm \Omega \pm N \pm 11) / t} \\
& \left.=\longrightarrow\left(1-\eta_{\Omega}^{2}\right)^{K(Z \pm \Omega \pm N) / t}\{0,2\}^{K(Z \pm \Omega \pm N) / t}\left\{D_{0 \Omega}\right\}\right]^{K(Z \pm \Omega \pm N) / t} \\
& \left(1-\eta_{\Omega}^{2}\right)^{K(Z \pm \Omega \pm N \pm H \pm P) / t}=\sum^{K(Z \pm \Omega \pm H)}{ }_{i=( \pm N \pm p)}\left(1-\eta_{\Omega}^{2}\right)^{K(Z \pm \Omega \pm N \pm H \pm P) / t}
\end{aligned}
$$

among them: $\left.\{0\}^{K(Z \pm \Omega \pm N \pm H \pm P) / t}\left\{D_{0 \Omega}\right\}\right]^{K(Z \pm \Omega \pm N \pm H \pm P) / t}$ Belong to spin, zero point mutation, transition state;

$$
\left.\{2\}^{K(Z \pm \Omega \pm N \pm H \pm P) / t}\left\{D_{0 \Omega}\right\}\right]^{K(Z \pm \Omega \pm N \pm H \pm P) / t} \text { Belong to sports and radiation }
$$

The circular logarithmic equation proves that under the entanglement, the spin state and the motion state are interdependent and mutually constrained.

The topological isomorphism, reciprocity, privacy, and boundary of the logarithm of the circle make various nonlinear combinations, exchanges, and sets in the boundary of the universe (clusters, galaxies, planets, and microscopic particles) normalized to Multi-element 1-1 linear combination. That is to say, as long as the interaction of any two individual star clusters, galaxies, planets, and microscopic particles in the whole universe is observed, the rules of change of the whole universe can be derived. According to this, it can be proved that Newton has universal gravitation - Coulomb has the scientific nature of electromagnetic force. On the contrary, it is also established

If applied to quantum bit and architecture chip development, the calculation program will be greatly simplified, which is conducive to the realization of high efficiency, multi-function, loss reduction, accuracy, security, boundary, privacy, openness and equality of quantum computers.The circular logarithmic equation proves that under the entanglement, the spin state and the motion state are interdependent and mutually constrained.

\subsection{Calculation of the cosmological equation $\Omega$}

Let: the universe calculus square $\Omega$ calculation is a 5 -dimensional (inactive material) +6 -dimensional (active material) composed of parallel/serial 11-dimensional equilibrium equation; $D_{\Omega}=D_{\Omega 5}+D_{\Omega 6}=243+45045$

$$
\begin{aligned}
& W_{\Omega}\{R\}=A x^{K(Z \pm \Omega \pm N \pm 0) / t}+B x^{K(Z \pm \Omega \pm N \pm 1) / t}+C x^{K(Z \pm \Omega \pm N \pm 2) / t}+\ldots \\
& +P x^{K(Z \pm \Omega \pm N \pm p) / t}+\ldots+Q x^{K(Z \pm \Omega \pm N \pm q) / t} \pm D_{\Omega}
\end{aligned}
$$

Power function: $(S=\Omega)=K(\Omega \pm N \pm 11) / t=K(\Omega \pm N \pm 5 \pm 6) / t$

$$
\begin{aligned}
& \left(1-\eta_{\Omega H^{2}}\right)^{\mathrm{K}(Z \pm \Omega \pm N \pm 11) / \mathrm{t}}=(1 / 2)\left[\left(1-\eta_{5 H^{2}}\right)^{\mathrm{K}(Z \pm \Omega \pm N \pm 5) / \mathrm{t}}+\left(1-\eta_{6 H^{2}}\right)^{\mathrm{K}(Z \pm \Omega \pm N \pm 6) / \mathrm{t}}\right] \\
& \left(1-\eta_{5 H^{2}}\right)^{\mathrm{K}(Z \pm \Omega \pm N \pm 5) / \mathrm{t}}=(1 / 5)[1+2+3+4+5] / 15=\Sigma\left(\eta_{H 5}\right)^{\mathrm{K}(Z \pm \Omega \pm N \pm 5)}=1 ; \\
& \left(1-\eta_{6 H^{2}}\right)^{\mathrm{K}(Z \pm \Omega \pm N \pm 5) / \mathrm{t}}=(1 / 6)[3+3+5+7+11+13] / 42=\Sigma\left(\eta_{H 6^{2}}\right)^{\mathrm{K}(Z \pm \Omega \pm N \pm 6)}=1 ;
\end{aligned}
$$

(1) Discrete five-dimensional equation $D_{5 \Omega}=3^{5}=243$;

$$
\left[X_{0} \pm D_{5}\right]^{K(Z \pm \Omega \pm N \pm 5)} ; D_{05 \Omega}=3,
$$




$$
\begin{aligned}
& \left(1-\eta^{2}\right)^{K(Z \pm \Omega \pm N \pm 6) / t}=\left\{\left(\sqrt{ } D_{5 \Omega}\right) /\left(D_{05 \Omega}\right)\right\}^{K(Z \pm \Omega \pm N \pm 6) / t}=243 / 243=1 ; \\
& {[X \pm D]^{K(Z \pm \Omega \pm N \pm 5) / t}=\left(1-\eta_{\Omega 5}{ }^{2}\right)^{K(Z \pm S \pm N \pm 5) / t}\left[X_{0} \pm D_{05}\right]^{K(Z \pm \Omega \pm N \pm 5) / t}} \\
& =\left(1-\eta_{\Omega 5}{ }^{2}\right)^{K(Z \pm \Omega \pm N \pm 6) / t}(0,2)^{K(Z \pm \Omega \pm N \pm 6) /}\{3\}^{K(Z \pm \Omega \pm N \pm 6) /} ;
\end{aligned}
$$

(2) Entangled six-dimensional equation;Entangled six-dimensional equation;Entangled six-dimensional equation; $\mathbf{D}_{6 \Omega}=\mathbf{7}^{6}=\mathbf{1 1 7 6 4 9}$;

已知条件: $\left[X_{0} \pm D_{6}\right]^{K(Z \pm \Omega \pm N \pm 6) / t} ; D_{06}=7, D_{6 \Omega}=45045,\left\{D_{0.06}\right\}^{(6) / t}=117649$

$$
\begin{aligned}
\left(1-\eta_{\Omega 6}{ }^{2}\right)^{K(Z \pm \Omega \pm N \pm 6) / t} & =\left\{\left({ }^{6} \sqrt{ } D_{6}\right) /\left(D_{06}\right)\right\}^{K(Z \pm \Omega \pm N \pm 6) / t}=45045 / 117649 \neq 1 ; \\
{[X \pm D]^{K(Z \pm \Omega \pm N \pm 6) / t} } & =\left(1-\eta_{\Omega 6}\right)^{K(Z \pm \Omega \pm N \pm 6) / t}\left[X_{0} \pm D_{06}\right]^{K(Z \pm \Omega \pm N \pm 6) / t}
\end{aligned}
$$

得:

$$
=\left(1-\eta_{\Omega 6}{ }^{2}\right)^{K(Z \pm \Omega \pm N \pm 6) / t}(0,2)^{K(Z \pm \Omega \pm N \pm 6) / t}\{7\}^{K(Z \pm \Omega \pm N \pm 6) / t} ;
$$

(3) $\Omega=11$-dimensional combination function-circle logarithmic equation $\left(1-\eta_{\Omega 11}\right)^{\mathrm{K}(Z \pm \Omega \pm N) / t}$

$$
\begin{aligned}
& {\left[X_{\Omega} \pm D_{\Omega}\right]^{K(Z \pm \Omega \pm N) / t}=\left[X_{5} \pm D_{5}\right]^{K(Z \pm \Omega \pm N \pm 5) / t}+\left[X_{6} \pm D_{6}\right]^{K(Z \pm \Omega \pm N \pm 6) / t}} \\
& \quad=\left[\left(1-\eta_{5 \Omega}{ }^{2}\right)(0,2)\{3\}\right]^{K(Z \pm \Omega \pm N \pm 5) / t}+\left[\left(1-\eta_{6 \Omega}{ }^{2}\right)(0,2)\{7\}\right]^{K(Z \pm \Omega \pm N \pm 6) / t} \\
& \quad=\left(1-\eta_{11 \Omega}{ }^{2}\right)^{K(Z \pm \Omega \pm N \pm 11) / t}\left(0,2^{11}\right)\left\{3^{K 5}+7^{K 6}\right\} ;
\end{aligned}
$$

(4) $\Omega=11$-dimensional combination of the logarithm of the circle $\Omega=11$-dimensional combination of the logarithm of the circle $\left(1-\eta_{\Omega 11}^{2}\right)^{\mathrm{K}(\mathrm{Z} \pm \Omega \pm N) / t}$;

$$
\begin{aligned}
& \left(1-\eta_{\Omega 11}{ }^{2}\right)^{K(Z \pm \Omega \pm N) / t}=\left[D_{5}+D_{6}\right] /\left[D_{05}{ }^{5}+D_{06}{ }^{6}\right] \\
& \left.=\left\{\left[{ }^{K 5} \sqrt{ } D / D_{05}\right]^{K(Z \pm 5 \pm N) / t}+\left[{ }^{K 6} \sqrt{ } D\right] / D_{06}\right]\right\}^{K(Z \pm 6 \pm N) / t} \\
& =\left[\left(1-\eta_{5}{ }^{2}\right)+\left(\left(1-\eta_{6}{ }^{2}\right)\right] ;\right.
\end{aligned}
$$

(5) Round logarithmic mean of 11-dimensional cosmological equation:

$$
D_{5 \Omega}+D_{6 \Omega}=243+117649=117892
$$

heve: $\quad\left(\eta_{\Omega 11}{ }^{2}\right)=(1 / 2)\left[\left(1-\eta_{5}{ }^{2}\right)+\left(\left(1-\eta_{6}{ }^{2}\right)\right] ;\left(\eta_{\Omega 11}\right)=(1 / 2)\left[\left(\eta_{5}\right)+\left(\left(\eta_{6}\right)\right] ;\right.\right.$

Solve: Unknown element value:

Order: unit circle logarithm $\left(1-\eta_{\Omega H}{ }^{2}\right)^{(Z \pm \Omega \pm N) / t}=\left(1-\eta_{\Omega}{ }^{2}\right)^{(Z \pm \Omega \pm N) / t}=1$;

(A): $\left(D_{0 \Omega}=3\right)\left(1-\eta_{\Omega H}{ }^{2}\right)^{(Z \pm \Omega \pm N \pm 5)}=D_{0 \Omega}{ }^{5}=243 \quad$, The five-dimensional equation is obtained as five point unit. The natural number: $(1,2,3,4,5)=15$ simulates the inactive neutral matter that is composed of discrete states.

(B): $\quad\left(D_{0}=7\right)\left(1-\eta_{\Omega H}{ }^{2}\right)^{K(Z \pm \Omega \pm N \pm 6) / t}=D_{0}^{6}=117649 ; \quad$ The six-dimensional equation is obtained as six point unit. The prime number is $(3,3,5,7,11,13)=42$

Its property power function, 
When: $\mathrm{K}=( \pm 0)$ is a neutral photon force calculation;

When: $\mathrm{K}=(-1)$ is a negative macroscopic electromagnetic force, micronuclear weak force, thermal entropy reduction calculation;

When: $\mathrm{K}=(+1)$ is a positive macroscopic gravitational force, micronuclear power, and thermal entropy increase calculation.

(C), Digital simulation calculation

(1) First energy expansion value Mass-to-energy ratio: ratio of bright matter to dark energy (astronomical observation data);

Ming matter (gravitational mass)

Dark energy

$$
\begin{aligned}
\{2\} \times\left[D_{05}+D_{06}\right] & :\left(13^{6}-235784\right) \\
& =235784: 4591012 \\
& =4.88488 \%: 95.11519 \%
\end{aligned}
$$

(2) Second energy expansion value

Material ratio: ratio of bright matter to dark matter (astronomical observation data: $68.5 \%$, simulation calculation $74 \%$ )

Ming matter (gravitational mass) $(2) \times\left[D_{05}+D_{06}\right]=235784$;

Dark matter

$$
\begin{aligned}
(2) \times\left[D_{05}+D_{06}\right]:\left(11^{6}-235789\right) \\
=235784: 935772 \\
=20.012 \%: 79.8739 \%
\end{aligned}
$$

(3) First mass compression value

$$
\left[D_{05}+D_{06}\right]=117892 \rightarrow\left(5^{6}-3^{5}\right)=15625-243 ;
$$

Mass compression value

117892:15625=7.664283:1

(4) Second quality compression value

$$
\left[D_{05}+D_{06}\right]=117892 \rightarrow\left(3^{6}-3^{5}\right)=729-243 ;
$$

Mass compression value

$$
117892: 486=242.576132: 1
$$

(5) Energy ratio: the ratio of bright energy to dark energy (high energy particle collision test data) Bright energy (gravitational mass)Bright energy (gravitational mass)

$$
\left\{2 \left\{\times\left[D_{05}+D_{06}\right]=235784\right.\right.
$$




$$
\begin{aligned}
& \text { Dark energy }\left(1-\eta_{\Omega 6^{2}}\right)^{-(z / t)}\left[D_{06}\right]=\left(13^{6}-235789\right) \\
& \begin{aligned}
&\{2\} \times\left[D_{05}+D_{06}\right]:(1771561-235789) \\
&= 117649: 4826809 \\
&=1: 41.02720 ;
\end{aligned}
\end{aligned}
$$

(5)Binomial calculation results: Astronomical observation, high-energy particle collision test data and digital simulation calculations are strikingly consistent. (2)(3)(4) is subject to negotiation.

\subsection{The quality of the universe can evolve}

The mass-energy of the universe evolves under the principle that the total energy $E_{\Omega}=\left(1-\eta_{\Omega}{ }^{2}\right)\left\{D_{\Omega}\right\}$ of the universe $\{\Omega\}$ is constant:

Respectively: $\rightarrow$ Convergence Pressure vortex (black hole) $(\mathrm{K}=+1) \rightarrow$ thermal nuclear fission of vacuum zero (hot wormhole) $(\mathbf{K}=+\mathbf{0}) \rightarrow$ expanded repulsion vortex (white hole) $(\mathrm{K}=-1) \rightarrow$ vacuum zero Cold nuclear fusion (cold wormhole)(K=-0) $\rightarrow$ Convergent pressure vortex (black hole) $(\mathrm{K}=+1) \rightarrow \cdots\}$, infinite loop of the universe.

The eigenmode is unchanged and written as a logarithmic form:Write a circular logarithmic evolution form

$$
\begin{aligned}
& \left\{\ldots \rightarrow\left(1-\eta_{\Omega}^{2}\right)^{+K(Z \pm \Omega \pm N) / t} \rightarrow\left(1-\eta_{\Omega}^{2}\right)^{0 K(Z \pm \Omega \pm N) / t} \rightarrow\left(1-\eta_{\Omega}^{2}\right)^{-K(Z \pm \Omega \pm N) / t} \rightarrow\right. \\
& \left.\ldots \rightarrow\left(1-\eta_{\Omega}^{2}\right)^{-K(Z \pm \Omega \pm N) / t} \rightarrow\left(1-\eta_{\Omega}^{2}\right)^{0 K(Z \pm \Omega \pm N) / t} \rightarrow\left(1-\eta_{\Omega}{ }^{2}\right)^{+K(Z \pm \Omega \pm N) / t} \rightarrow \ldots\right\} ;
\end{aligned}
$$

In particular, under the entanglement, the spin state and the motion state are interdependent and mutually restrictive. That is to say, the spin state has a synchronous change with the (radiation, revolution) motion state.

\section{[Guess]}

(1), the minimum natural number $\{1,2,3,4,5\}=\{15\}=\{3,5\}$ constitutes the inactive characteristic modulus value $\left\{D_{05}=3\right\}$ and the smallest prime number $\{3,3,5,7,11,13\}=\{42\}$ constitutes the active characteristic modulus value $\left\{D_{06}=7\right\}$, which constitutes the universe (nature) parallel/serial basic feature mode. (1)

(2) $\{\mathbf{4 2}\}$ constitutes "active material ions", in the form of characteristic molds, vacuum excitation produces asymmetry (vortex, vortex ring) material-space, energy, expansion force, contraction force, called "parity is not conserved." It is considered by scientists to be the meaning of the existence of all life in the universe.

$$
\begin{aligned}
& \left(1-\eta_{\Omega 6}{ }^{2}\right)^{+(Z / t)} \cdot\left[D_{06}\right] \leq\left(1-\eta_{\Omega 6}{ }^{2}\right)^{0(Z / t)} \cdot\left[D_{06}\right] \leq\left(1-\eta_{\Omega 6}{ }^{2}\right)^{-(Z / t)} \cdot\left[D_{06}\right] ; \\
& \left(1-\eta_{\Omega 6}{ }^{2}\right)^{K(Z / t)}=\left[\left(1-\eta_{\Omega 6}{ }^{2}\right)^{0(Z / t)}+\left(1-\eta_{\Omega 6}{ }^{2}\right)^{-(Z / t)}+\left(1-\eta_{\Omega 6}{ }^{2}\right)\right]^{k(Z / t)}=\{2\}^{K(Z / t)} ; \\
& \left(1-\eta_{\Omega 6}{ }^{0}\right)^{(Z / / t)}=\left[\left(1-\eta_{\Omega 6}{ }^{2}\right)^{-(Z / t)}+\left(1-\eta_{\Omega 6}{ }^{2}\right)\right]^{k(Z / t)}=\{1\}^{K(Z / t)} ;
\end{aligned}
$$

\section{The conclusion}

The logarithm of the circle contains a lot of information, including the reform of traditional calculus, logarithm, the arithmetic of logical algebra, and the establishment of novel mathematical ideas. The functions of arbitrary uncertainty multi-element combination exchange sets in various scientific fields, as 
well as various algorithms such as topology, probability, chaos, fractal, etc., can be resolved into abstract circular logarithmic equations, and realize the irrelevant computational model in [0to1] Interval arithmetic operations.

$$
\text { Unified formula: } \quad W=\left(1-\eta^{2}\right)^{z / t} W_{0}
$$

Equation (28) describes the high degree of fit between circular logarithmic mathematics and natural rules.

Today, a small number of scholars continue to persistently explore the mysteries of nature understanding mathematics progress. The quantum computing giant is catching up with the production of "super quantum computers", and life science workers have encountered many mathematical difficulties in exploring "brain thinking calculations", "cell growth and immunity". The above problems become a new mathematical theorem of the circular logarithm by solving the century mathematics problem. The main proofs are: "Reciprocal Lemma", "Hodge Conjecture", "BSD Conjecture", "P=NP", "Riemann (Zero) Conjecture", "Regulatory Field", "NS Equation", etc. The puzzle becomes a new theorem. Ensure the scientific, innovative and reliable circular logarithm algorithm.

Circular logarithm is a good computational model that successfully deals with symmetry and asymmetry, discrete and entangled, parallel/serial computing, zero-point exchange (boundary), set, transition, and so on. Has superb computing power. Adapt to today's popular blockchain, artificial intelligence, life sciences, physical mathematics, mathematical mechanics, network transactions, information transmission, economic evaluation and other needs. (end)

\section{REFERENCES}

[1] [United States] M. Klein translated "Ancient and Modern Mathematical Thoughts" by Deng Dongjun (Vol. 1, 2, 3) Shanghai Science and Technology Press, August 2014

[2] "Langlanz Program", "100 scientific problems in the 21st century" p793-p798 Jilin People's Publishing House, January 2003, third edition

[3] Xu Lizhi, "Selection of Mathematical Methodology" p47-p101 Huazhong Institute of Technology Press, April 1983

[4] Yang Guilin and other editors "Modern Physics" p155-189 Beijing Science Press (www.sciencep.com) 2006 third print.

[5] John Deby Hill Chen Wei Peng translated "The Love of Prime Numbers - Riemann and the biggest unsolved fan in mathematics" Shanghai Science and Technology Education Press published in December 2008.

[6] John Stahlell "Einstein's Miracle Year - Five Papers on Changing Physics" Fan Yannian and other translations Shanghai Science and Technology Education Press, November 20012

[7] [United States] M. Livio, Huang Zheng, "Mathematic Meditations", Beijing People's Posts and Telecommunications Press, published in September 2011

[8] Wang Yiping, "On Light, Polynomial, Relativity, Circular Network, Isomorphic Mapping, Commemorating the 100th Anniversary of Einstein's General Theory of Relativity and 2015-International Light Year" 
[United States] "MATTER REGULARITY" (Gewu) 2015/3 p46 -61 (Chinese and English) Published in June 2015

[9] Wang Yiping, "NP-P and Relativistic Structure" [United States] Journal of Mathematics and Statistics Science (JCCM) 2018/9 p1-14 September 2018

[10] Wang Yiping, "Riemann Function and Relativistic Structure" [United States] Journal of Mathematics and Statistics Science (JMSS) 2018/1 p55-67 2018.1 months

[11] Wang Yiping, "NS Equation and Relativistic Structure" [United States] Journal of Mathematics and Statistics Science (JCCM) 2018/5 p55-67 May 2018

[12] Wang Hongxuan "Four-color theorem based on circular logarithm-polynomial analysis" [United States] Journal of Mathematics and Statistics Science (JCCM) 2018/9 p15-29 2018September edition 